\title{
Probing Cyanocuprates by Electrospray Ionization Mass Spectrometry
}

\author{
Aliaksei Putau and Konrad Koszinowski* \\ Department Chemie, Ludwig-Maximilians-Universität München, Butenandtstr. 5 - 13, \\ 81377 München, Germany \\ Supporting Information
}

\section{Table of Contents}

1.) Anion-mode ESI mass spectra recorded with the TSQ 7000 instrument (Figures S1 - S4)

2.) Characterization of the HCT ion trap (Figures S5 - S8)

3.) Comparison of measured and simulated isotope patterns (Figures S9-S10)

4.) Additional anion-mode ESI mass spectra of $\mathrm{LiCuR}_{2} \cdot \mathrm{LiCN}$ solutions (Figures S11 - S15)

5.) Additional anion-mode ESI mass spectra of $\mathrm{LiCu}(\mathrm{Me}) \mathrm{R} \cdot \mathrm{LiCN}$ solutions (Figures S16 - S26)

6.) Additional anion-mode ESI mass spectra of $\operatorname{LiCuR}(\mathrm{CN})$ solutions (Figures S27 - S33)

7.) Additional cation-mode ESI mass spectra of $\mathrm{LiCuR}_{2} \cdot \mathrm{LiCN}$ solutions (Figures S34 - S38)

8.) Additional cation-mode ESI mass spectra of $\mathrm{Li}_{0.8} \mathrm{CuR}_{0.8}(\mathrm{CN})$ solutions

(Figures S39 - S44)

9.) Mass spectra of mass-selected $\mathrm{Li}_{n-1} \mathrm{Cu}_{n} \mathrm{R}_{2 n}{ }^{-}$anions and their fragment ions (Figures S45 - S56)

10.) Mass spectra of mass-selected $\mathrm{Li}_{n-1} \mathrm{Cu}_{n} \mathrm{Me}_{2 n-x} \mathrm{R}_{x}{ }^{-}$anions and their fragment ions (Figures S57 - S72)

11.) Mass spectra of mass-selected cyanide-containing cuprate anions and their fragment ions (Figures S73 - S100) 


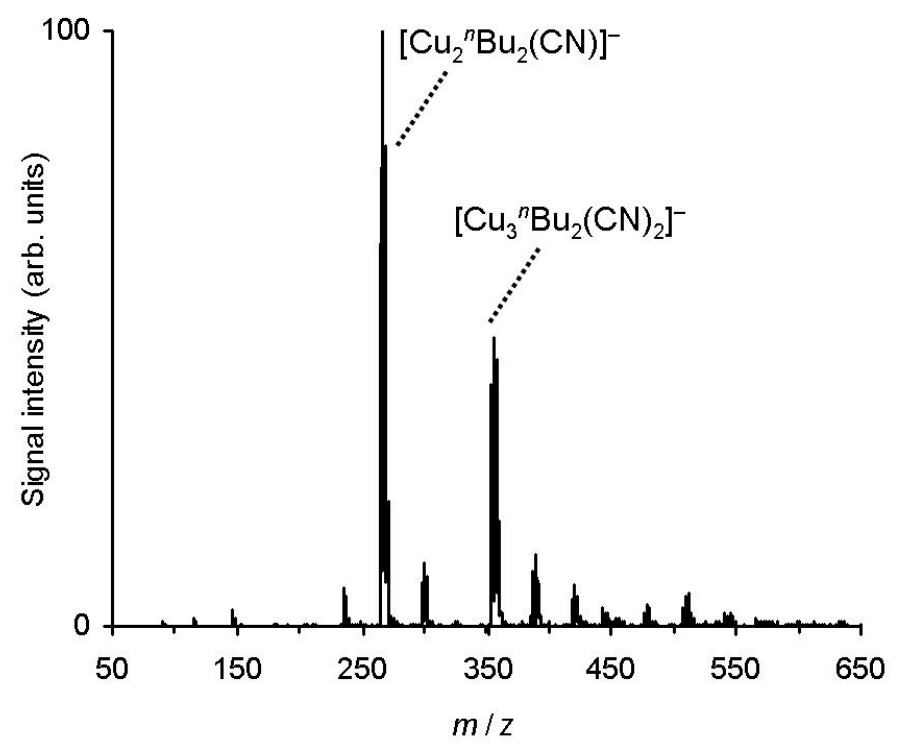

Figure S1. Anion-mode ESI mass spectrum of a $25 \mathrm{mM}$ solution of $\mathrm{LiCu}^{n} \mathrm{Bu}_{2} \cdot \mathrm{LiCN}$ in $\mathrm{THF}$, measured with the TSQ 7000 instrument.

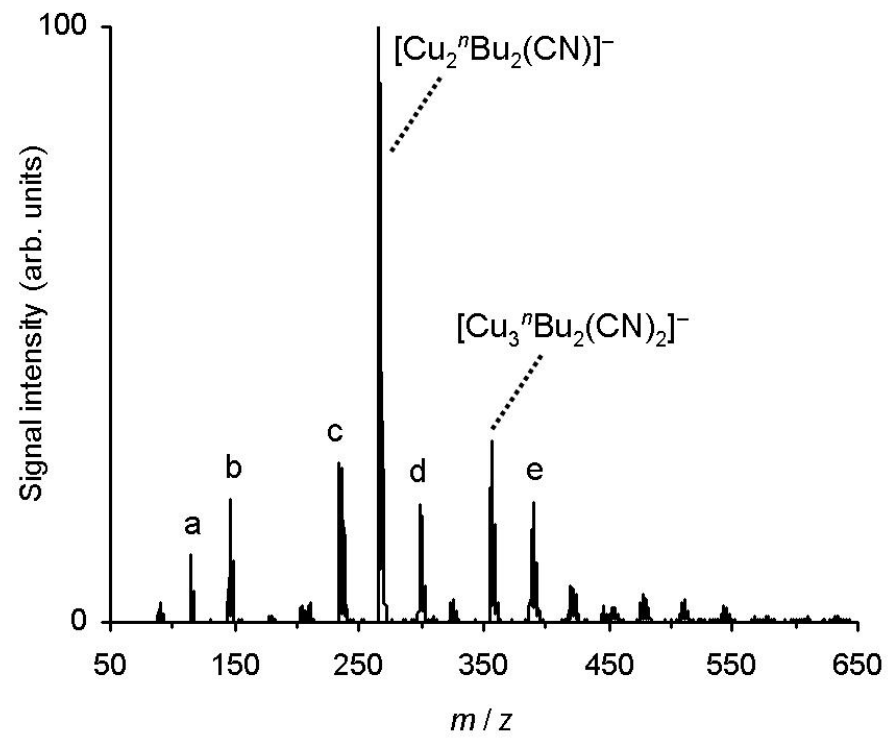

Figure S2. Anion-mode ESI mass spectrum of a $25 \mathrm{mM}$ solution of $\mathrm{LiCu}^{n} \mathrm{Bu}(\mathrm{CN})$ in $\mathrm{THF}$, measured with the TSQ 7000 instrument, $\mathrm{a}=\mathrm{Cu}(\mathrm{CN})_{2}{ }^{-}, \mathrm{b}=\mathrm{Cu}^{n} \mathrm{Bu}(\mathrm{CN})^{-}, \mathrm{c}=\mathrm{Cu}_{2}{ }^{n} \mathrm{Bu}(\mathrm{CN})_{2}^{-}, \mathrm{d}=$ $\mathrm{LiCu}_{2}{ }^{n} \mathrm{Bu}_{2}(\mathrm{CN})_{2}{ }^{-}, \mathrm{e}=\mathrm{LiCu}_{3}{ }^{n} \mathrm{Bu}_{2}(\mathrm{CN})_{3}{ }^{-}$. 


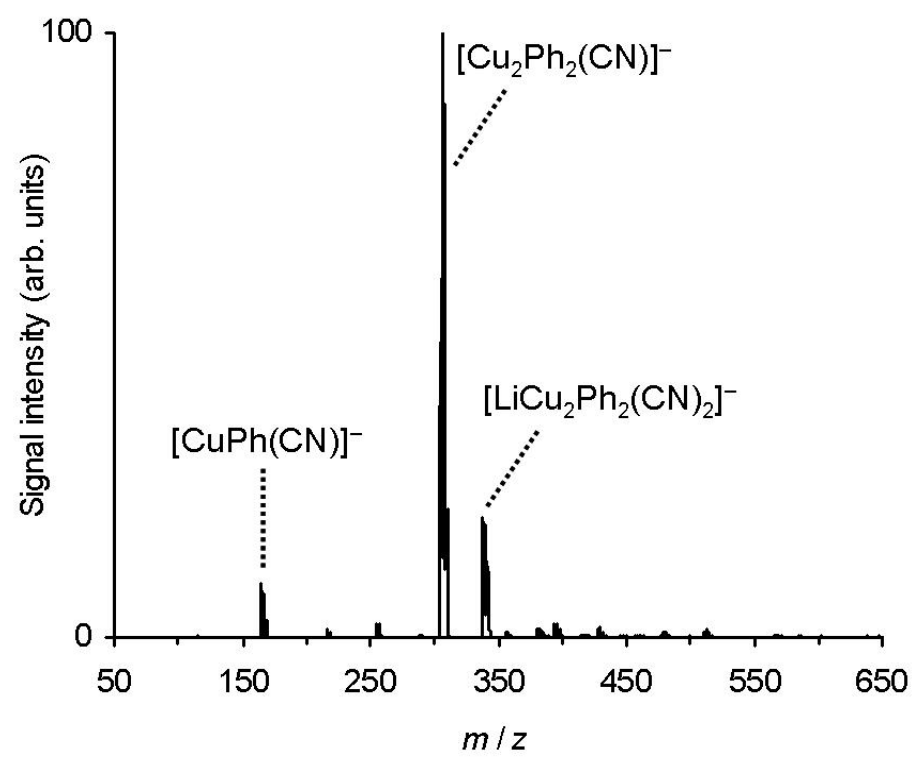

Figure S3. Anion-mode ESI mass spectrum of a $25 \mathrm{mM}$ solution of $\mathrm{LiCuPh}_{2} \cdot \mathrm{LiCN}$ in $\mathrm{THF}$, measured with the TSQ 7000 instrument.

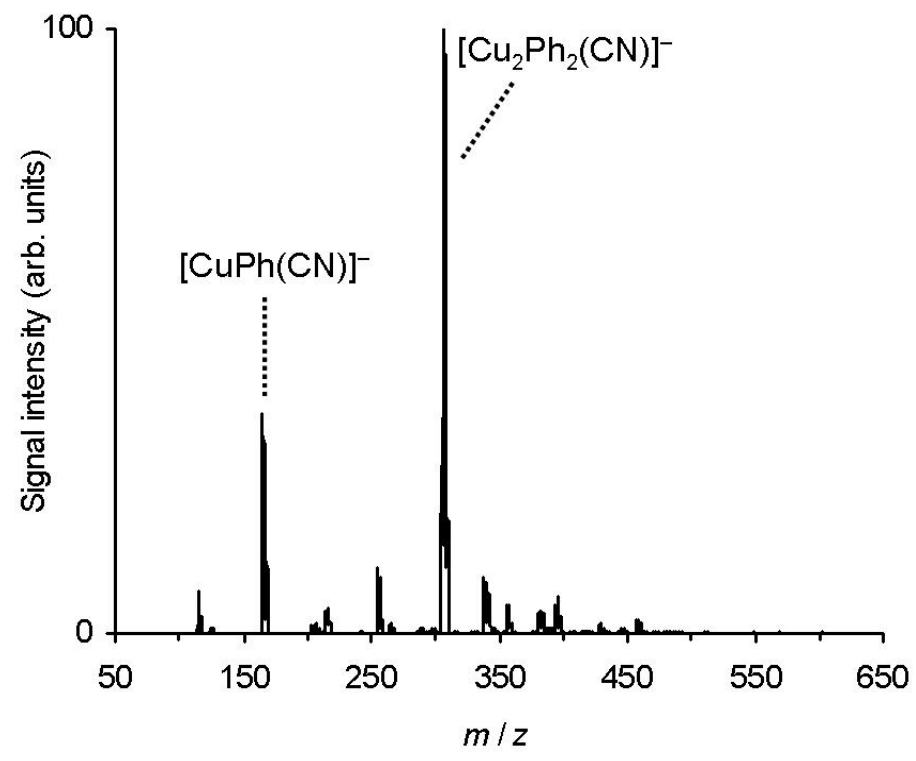

Figure S4. Anion-mode ESI mass spectrum of a $25 \mathrm{mM}$ solution of $\mathrm{LiCuPh}(\mathrm{CN})$ in $\mathrm{THF}$, measured with the TSQ 7000 instrument. 


\section{2.) Characterization of the $\mathrm{HCT}$ ion trap}

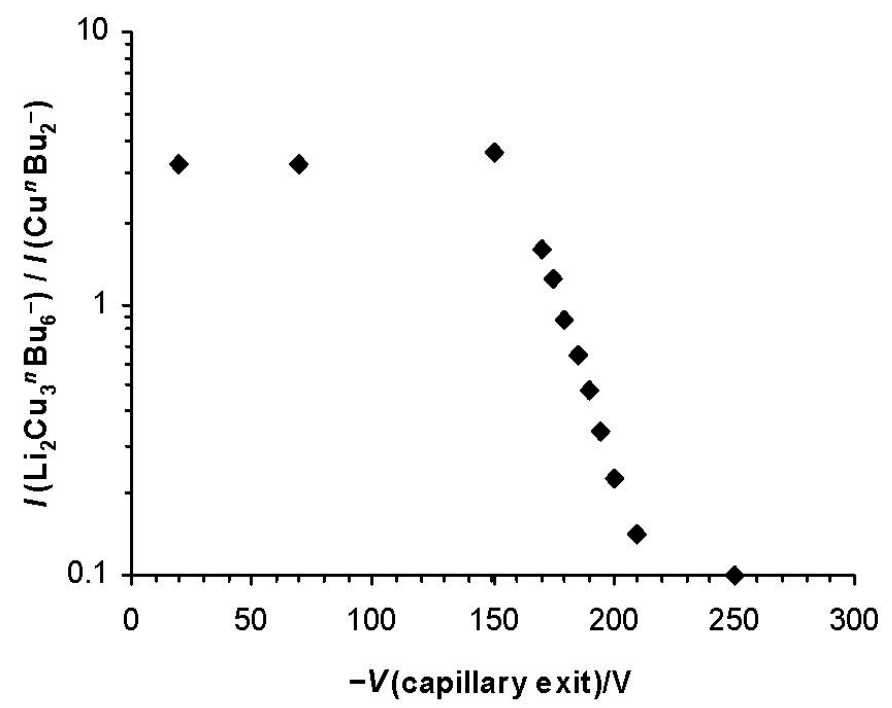

Figure S5. Ratio of the signal intensities of $\mathrm{Li}_{2} \mathrm{Cu}_{3}{ }^{n} \mathrm{Bu}_{6}{ }^{-}$and $\mathrm{Cu}^{n} \mathrm{Bu}_{2}{ }^{-}$produced by ESI of a 25 $\mathrm{mM}$ solution of $\mathrm{LiCu}^{n} \mathrm{Bu}_{2} \cdot \mathrm{LiCN}$ in THF as a function of the voltage of the capillary exit (other parameters: $V($ Oct $1 \mathrm{DC})=-5 \mathrm{~V}, V($ Oct $2 \mathrm{DC})=-1.7 \mathrm{~V}$, trap drive level of 20$)$.

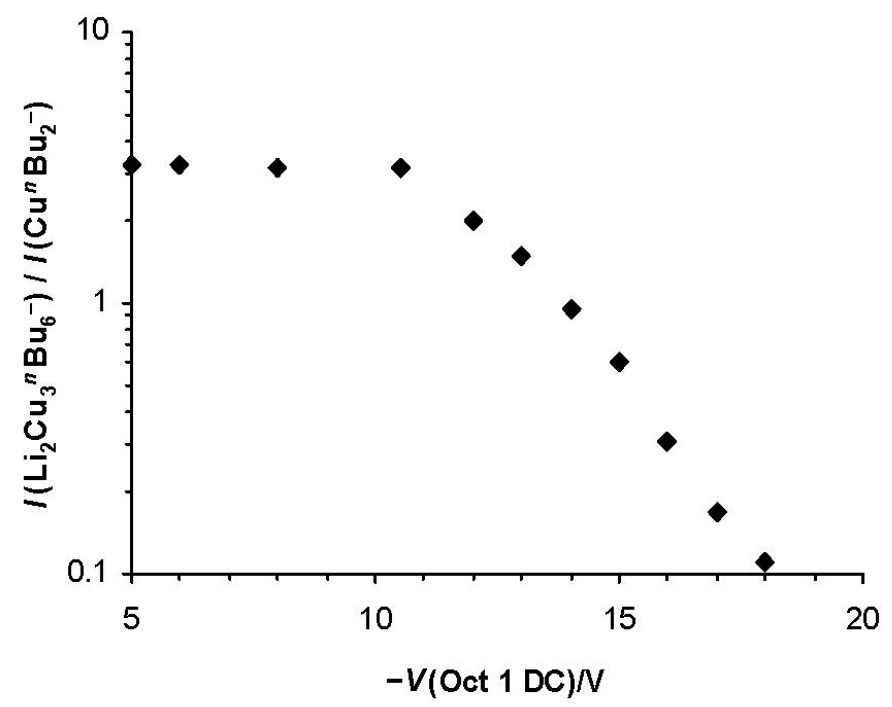

Figure S6. Ratio of the signal intensities of $\mathrm{Li}_{2} \mathrm{Cu}_{3}{ }^{n} \mathrm{Bu}_{6}{ }^{-}$and $\mathrm{Cu}^{n} \mathrm{Bu}_{2}{ }^{-}$produced by ESI of a 25 $\mathrm{mM}$ solution of $\mathrm{LiCu}^{n} \mathrm{Bu}_{2} \cdot \mathrm{LiCN}$ in THF as a function of the voltage of the first transfer octopole (other parameters: $V($ capillary exit $)=-20 \mathrm{~V}, V($ Oct $2 \mathrm{DC})=-1.7 \mathrm{~V}$, trap drive level of 20 ). 


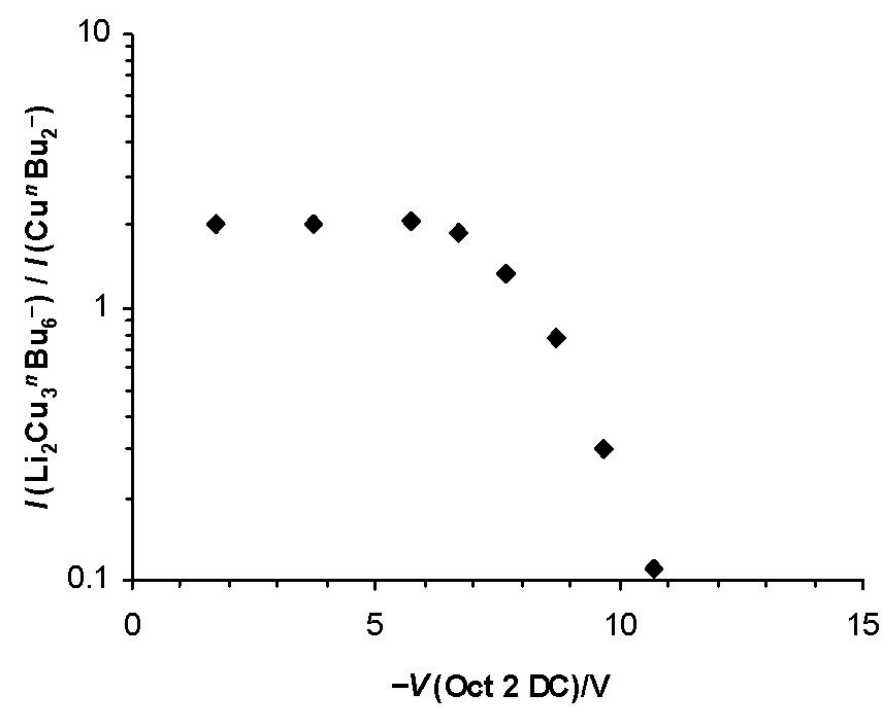

Figure S7. Ratio of the signal intensities of $\mathrm{Li}_{2} \mathrm{Cu}_{3}{ }^{n} \mathrm{Bu}_{6}{ }^{-}$and $\mathrm{Cu}^{n} \mathrm{Bu}_{2}{ }^{-}$produced by ESI of a 25 $\mathrm{mM}$ solution of $\mathrm{LiCu}^{n} \mathrm{Bu}_{2} \cdot \mathrm{LiCN}$ in THF as a function of the voltage of the second transfer octopole (other parameters: $V($ capillary exit $)=-20 \mathrm{~V}, V($ Oct $1 \mathrm{DC})=-12 \mathrm{~V}$, trap drive level of 20).

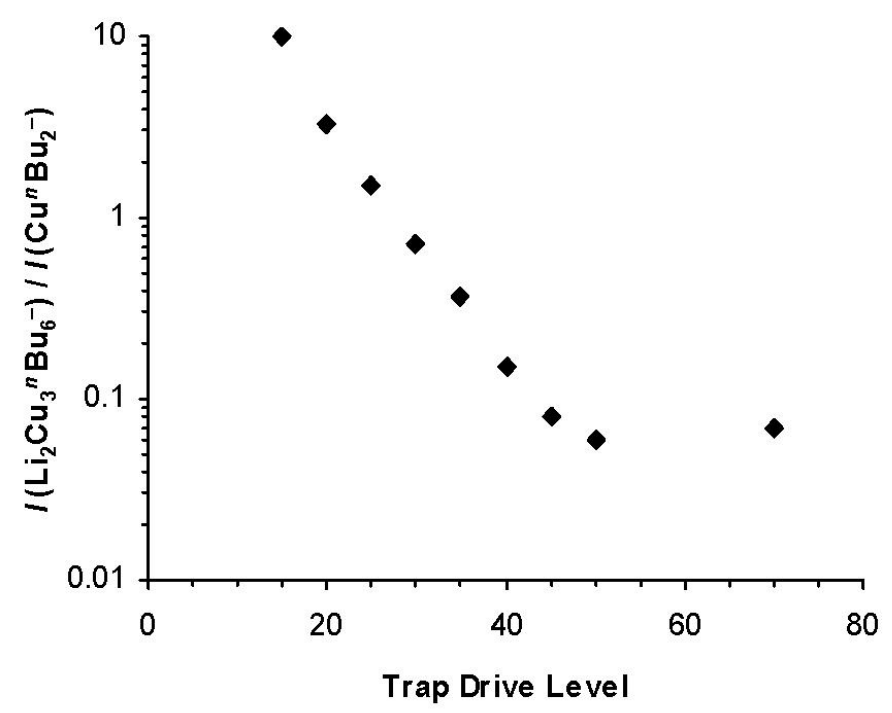

Figure S8. Ratio of the signal intensities of $\mathrm{Li}_{2} \mathrm{Cu}_{3}{ }^{n} \mathrm{Bu}_{6}{ }^{-}$and $\mathrm{Cu}^{n} \mathrm{Bu}_{2}{ }^{-}$produced by ESI of a 25 $\mathrm{mM}$ solution of $\mathrm{LiCu}^{n} \mathrm{Bu}_{2} \cdot \mathrm{LiCN}$ in THF as a function of the trap drive level (other parameters: $V($ capillary exit $)=-20 \mathrm{~V}, V($ Oct $1 \mathrm{DC})=-16 \mathrm{~V}, V($ Oct $2 \mathrm{DC})=-1.7 \mathrm{~V})$. 


\section{3.) Comparison of measured and simulated isotope patterns}

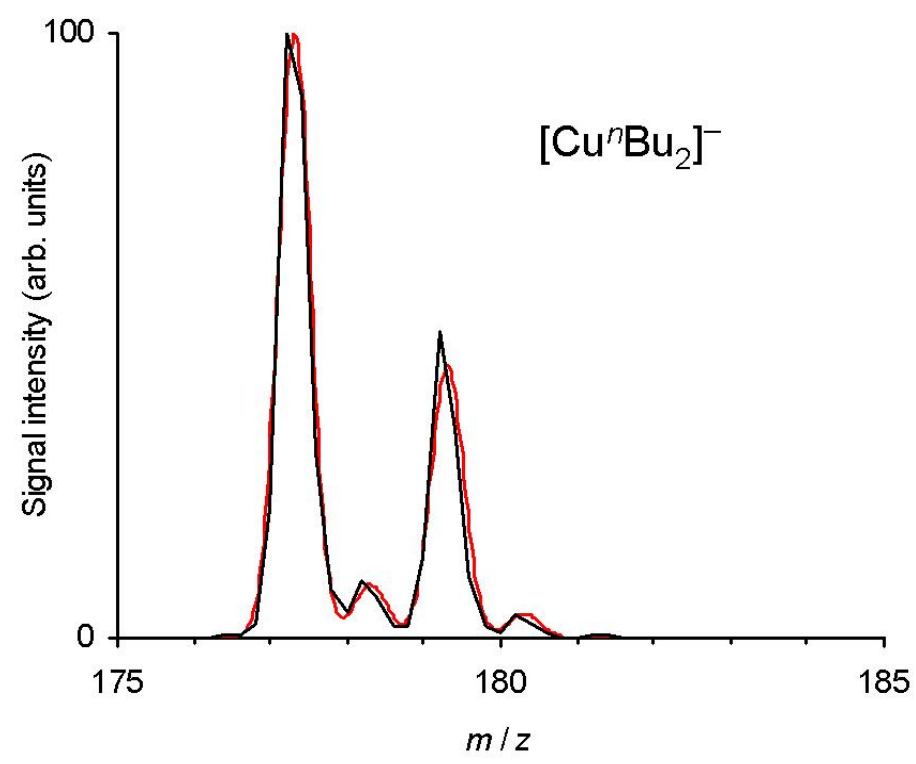

Figure S9. Comparison of observed (black) and simulated (red) isotope patterns for $\mathrm{Cu}^{n} \mathrm{Bu}_{2}{ }^{-}$.

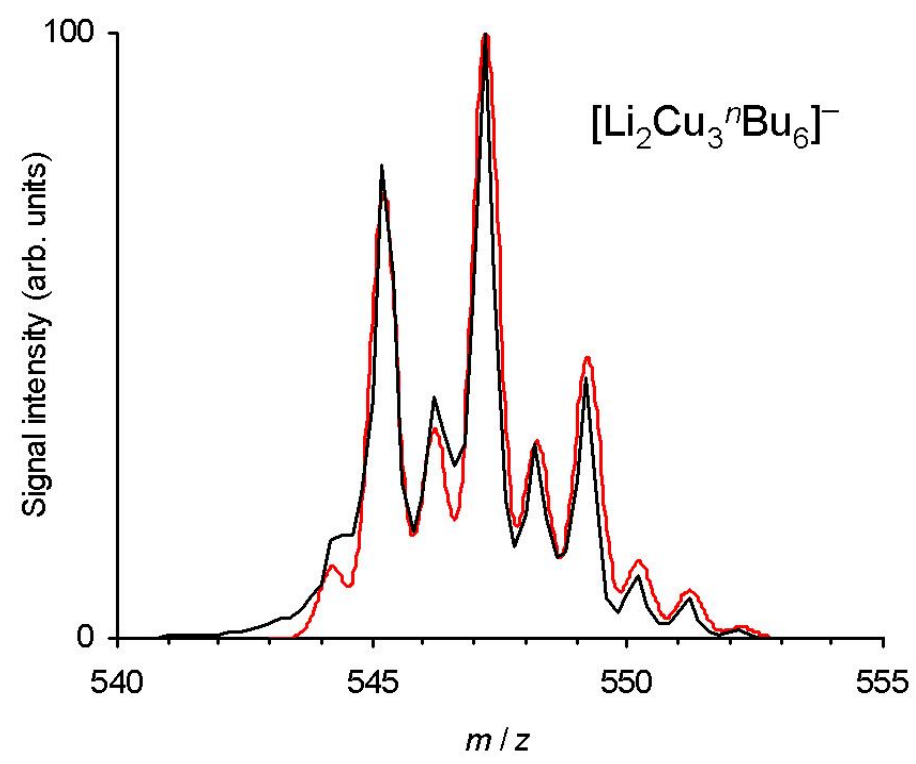

Figure S10. Comparison of observed (black) and simulated (red) isotope patterns for $\mathrm{Li}_{2} \mathrm{Cu}_{3}{ }^{n} \mathrm{Bu}_{6}{ }^{-}$ 


\section{4.) Additional anion-mode ESI mass spectra of $\mathrm{LiCuR}_{2} \cdot \mathrm{LiCN}$ solutions}

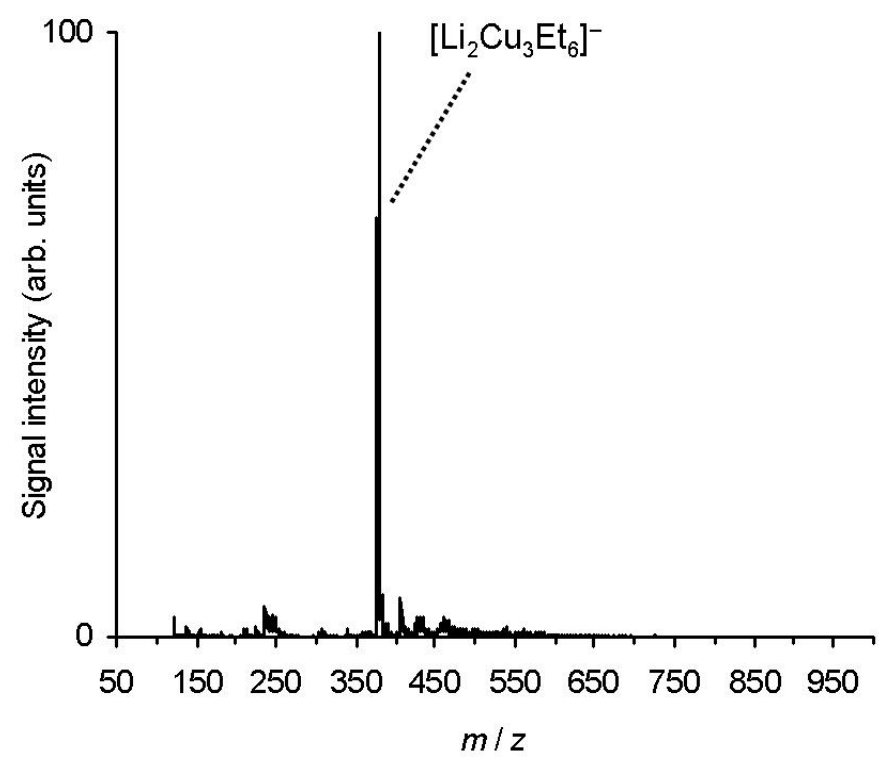

Figure S11. Anion-mode ESI mass spectrum of a $25 \mathrm{mM}$ solution of $\mathrm{LiCuEt}_{2} \cdot \mathrm{LiCN}$ in THF.

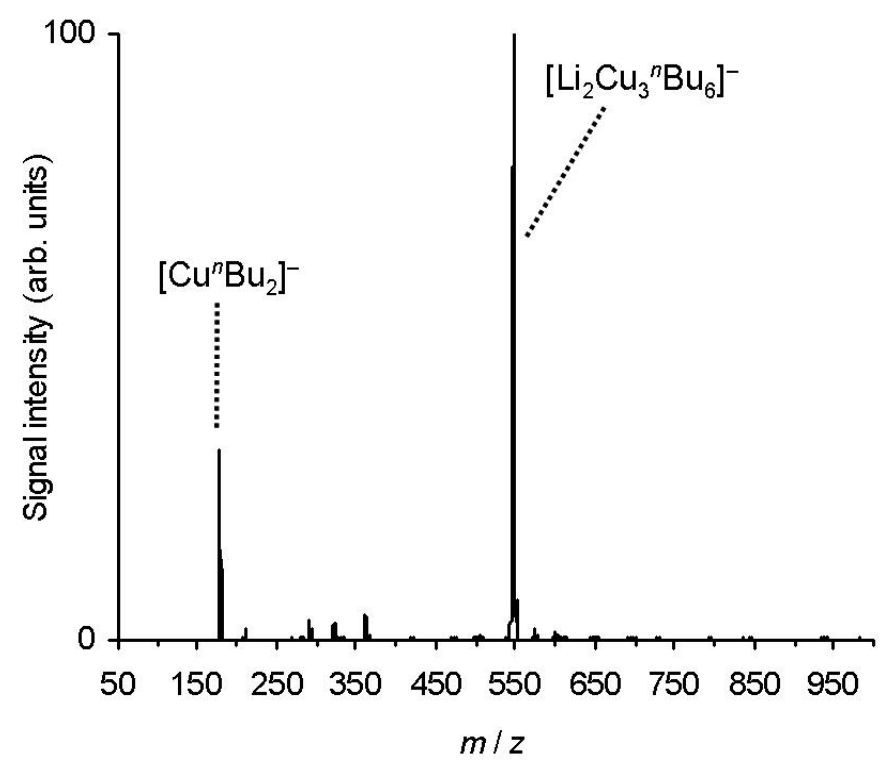

Figure S12. Anion-mode ESI mass spectrum of a $25 \mathrm{mM}$ solution of $\mathrm{LiCu}^{n} \mathrm{Bu}_{2} \cdot \mathrm{LiCN}$ in THF. 


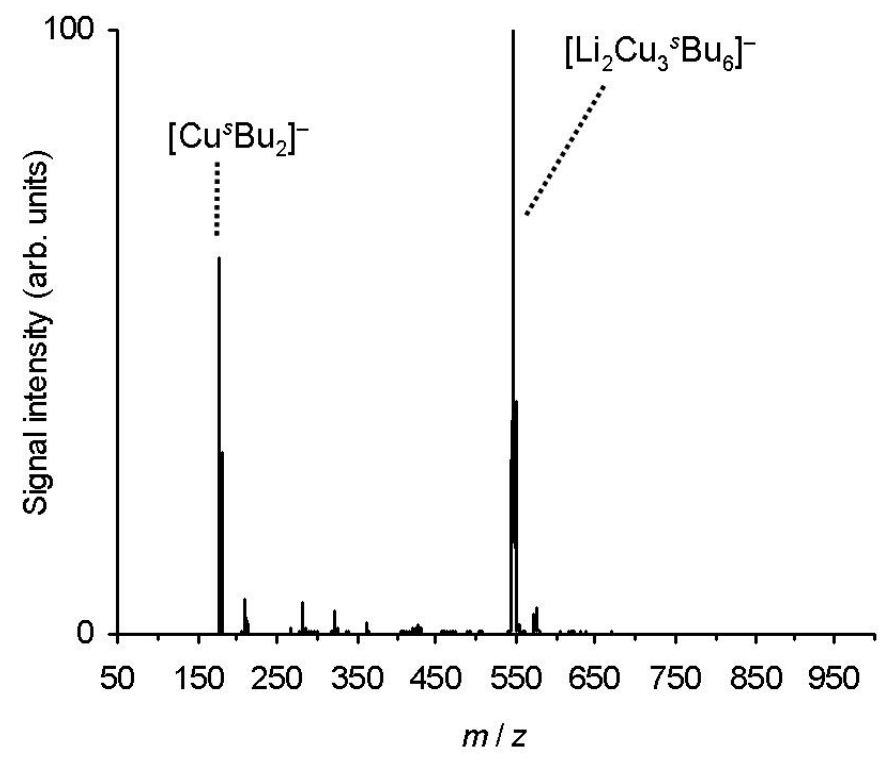

Figure S13. Anion-mode ESI mass spectrum of a $25 \mathrm{mM}$ solution of $\mathrm{LiCu}^{s} \mathrm{Bu}_{2} \cdot \mathrm{LiCN}$ in $\mathrm{THF}$.

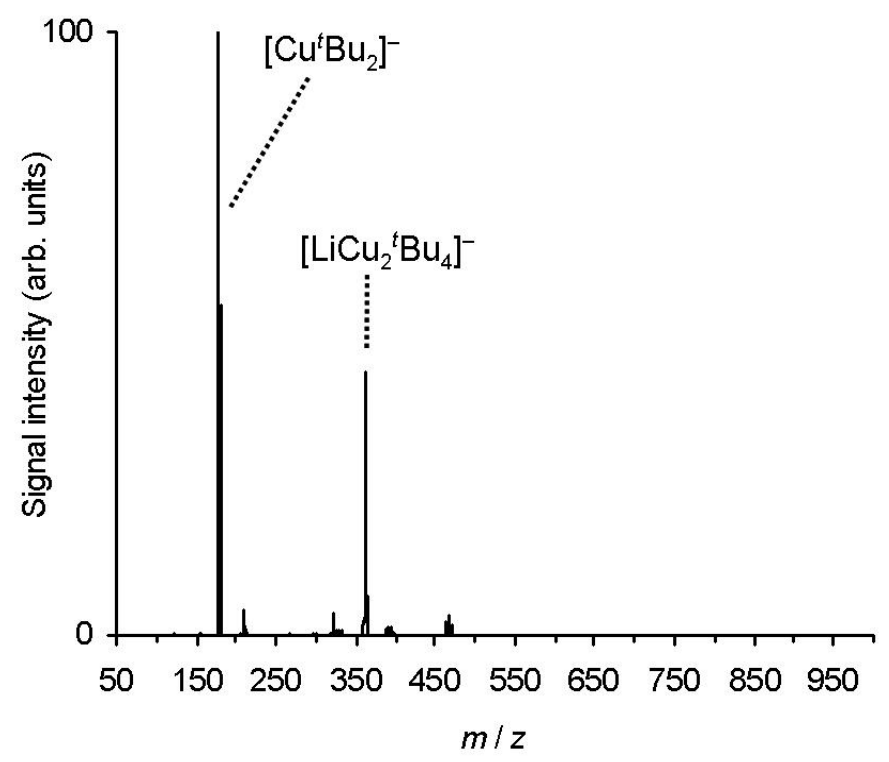

Figure S14. Anion-mode ESI mass spectrum of a $25 \mathrm{mM}$ solution of $\mathrm{LiCu}^{t} \mathrm{Bu}_{2} \cdot \mathrm{LiCN}$ in $\mathrm{THF}$. 


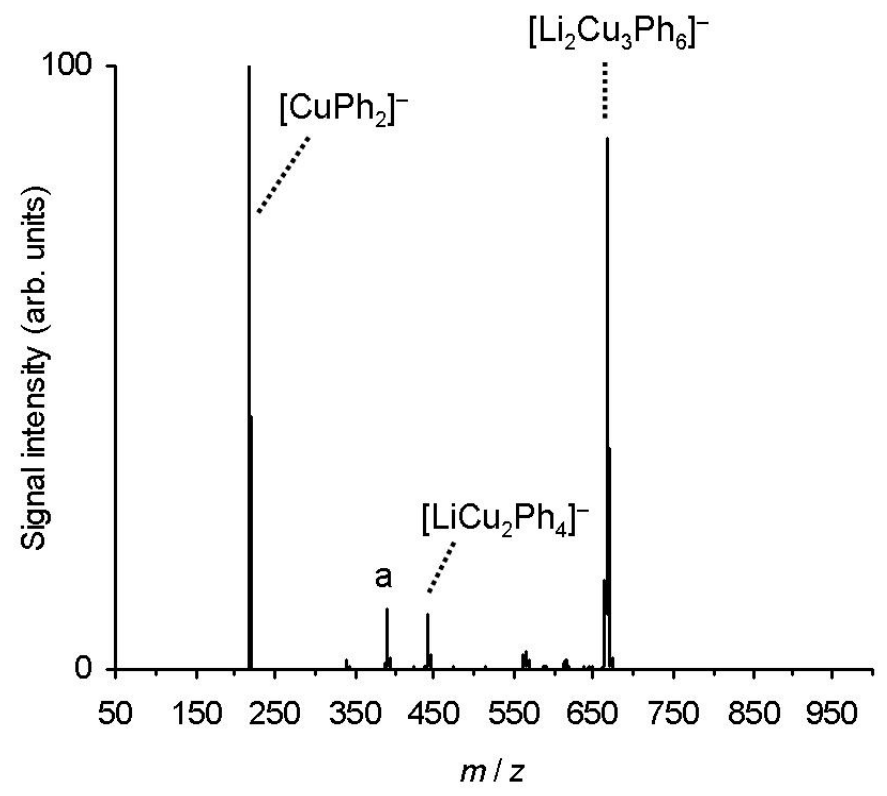

Figure S15. Anion-mode ESI mass spectrum of a $25 \mathrm{mM}$ solution of $\mathrm{LiCuPh}_{2} \cdot \mathrm{LiCN}$ in $\mathrm{THF}, \mathrm{a}=$ $\mathrm{LiCu}_{2} \mathrm{Ph}_{3}(\mathrm{CN})^{-}$. 


\section{5.) Additional anion-mode ESI mass spectra of $\mathrm{LiCu}(\mathrm{Me}) \mathrm{R} \cdot \mathrm{LiCN}$ solutions}

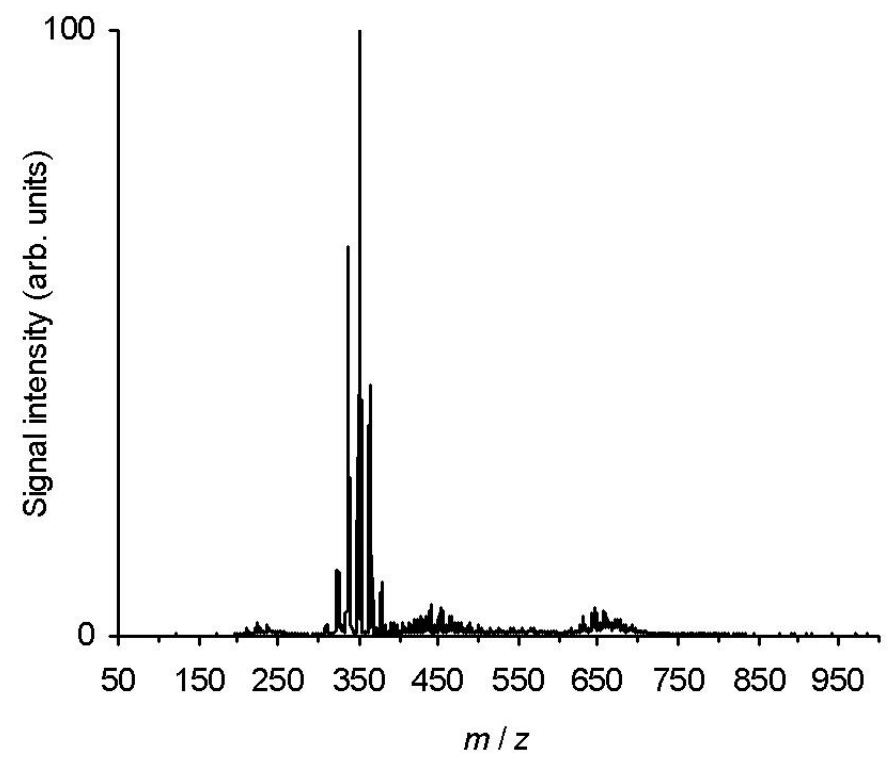

Figure S16. Anion-mode ESI mass spectrum of a $25 \mathrm{mM}$ solution of $\mathrm{LiCu}(\mathrm{Me}) \mathrm{Et} \cdot \mathrm{LiCN}$ in $\mathrm{THF}$.

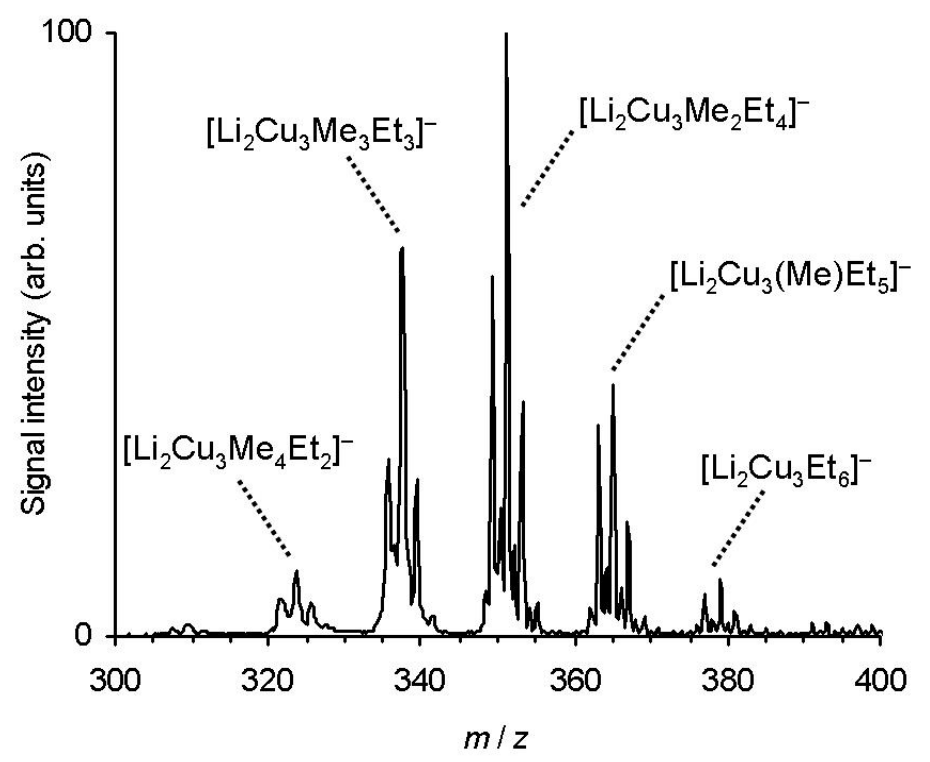

Figure S17. Section from the anion-mode ESI mass spectrum of a $25 \mathrm{mM}$ solution of $\mathrm{LiCu}(\mathrm{Me}) \mathrm{Et} \cdot \mathrm{LiCN}$ in THF. 


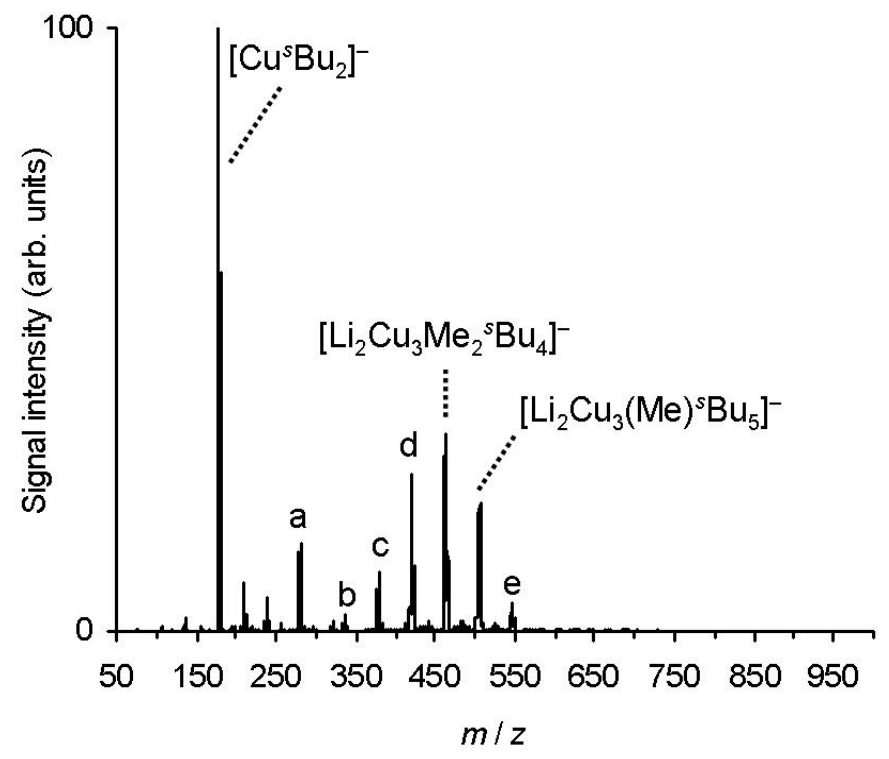

Figure S18. Anion-mode ESI mass spectrum of a $25 \mathrm{mM}$ solution of $\mathrm{LiCu}(\mathrm{Me})^{s} \mathrm{Bu} \cdot \mathrm{LiCN}$ in THF, $\mathrm{a}=\mathrm{LiCu}_{2} \mathrm{Me}(\mathrm{OH})^{s} \mathrm{Bu}_{2}{ }^{-} / \mathrm{LiCu}_{2}(\mathrm{OH})_{2}{ }^{s} \mathrm{Bu}_{2}^{-}, \mathrm{b}=\mathrm{Li}_{2} \mathrm{Cu}_{3} \mathrm{Me}_{5}{ }^{s} \mathrm{Bu}^{-}, \mathrm{c}=\mathrm{Li}_{2} \mathrm{Cu}_{3} \mathrm{Me}_{4}{ }^{s} \mathrm{Bu}_{2}{ }^{-}, \mathrm{d}=$ $\mathrm{Li}_{2} \mathrm{Cu}_{3} \mathrm{Me}_{3}{ }^{s} \mathrm{Bu}_{3}{ }^{-}, \mathrm{e}=\mathrm{Li}_{2} \mathrm{Cu}_{3}{ }^{s} \mathrm{Bu}_{6}{ }^{-}$.

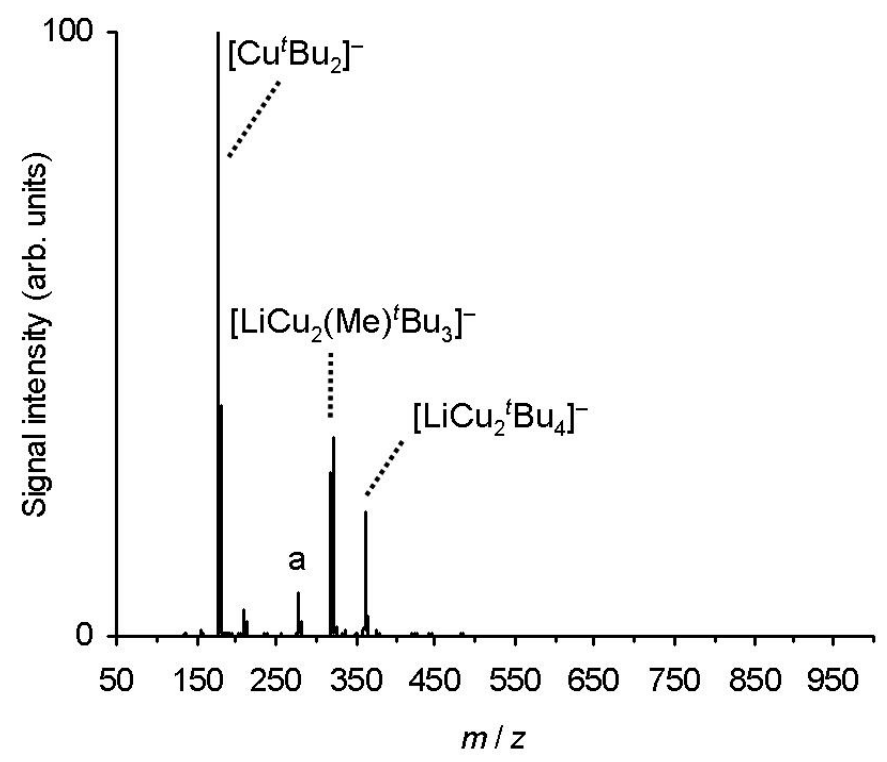

Figure S19. Anion-mode ESI mass spectrum of a $25 \mathrm{mM}$ solution of $\mathrm{LiCu}(\mathrm{Me})^{t} \mathrm{Bu} \cdot \mathrm{LiCN}$ in THF, $\mathrm{a}=\mathrm{LiCu}_{2} \mathrm{Me}_{2}{ }^{t} \mathrm{Bu}_{2}^{-}$. 


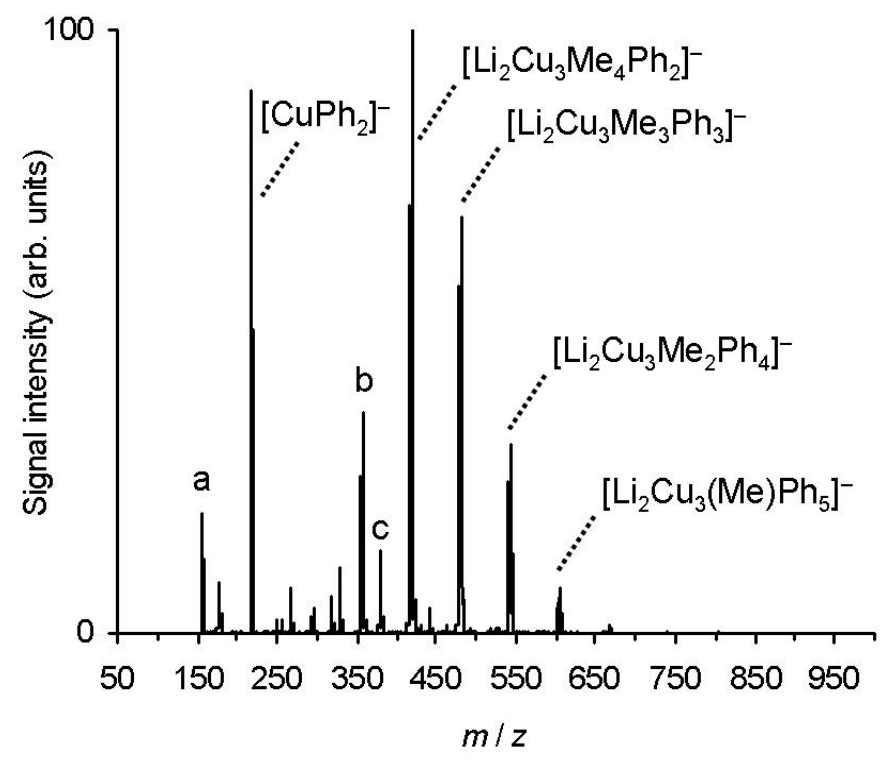

Figure S20. Anion-mode ESI mass spectrum of a $25 \mathrm{mM}$ solution of $\mathrm{LiCu}(\mathrm{Me}) \mathrm{Ph} \cdot \mathrm{LiCN}$ in THF, $\mathrm{a}=\mathrm{Cu}(\mathrm{Me}) \mathrm{Ph}^{-}, \mathrm{b}=\mathrm{Li}_{2} \mathrm{Cu}_{3} \mathrm{Me}_{5} \mathrm{Ph}^{-}, \mathrm{c}=\mathrm{LiCu}_{2}(\mathrm{Me}) \mathrm{Ph}_{3}{ }^{-}$.

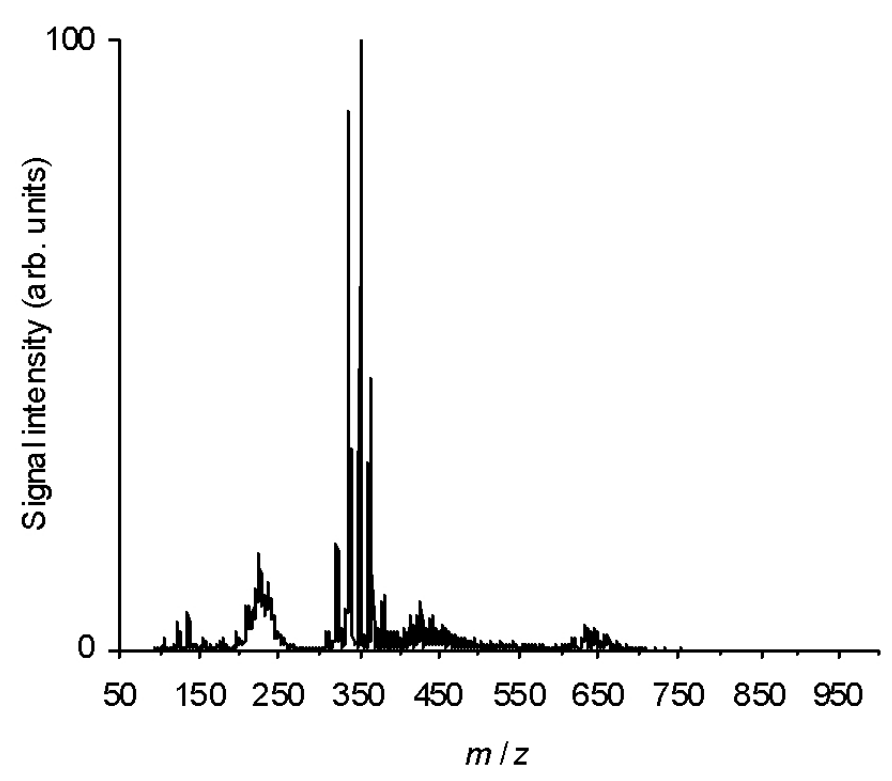

Figure S21. Anion-mode ESI mass spectrum of a 1:1 mixture of $25 \mathrm{mM}$ solutions of $\mathrm{LiCuMe}_{2} \cdot \mathrm{LiCN}$ and $\mathrm{LiCuEt}_{2} \cdot \mathrm{LiCN}$ in THF. 


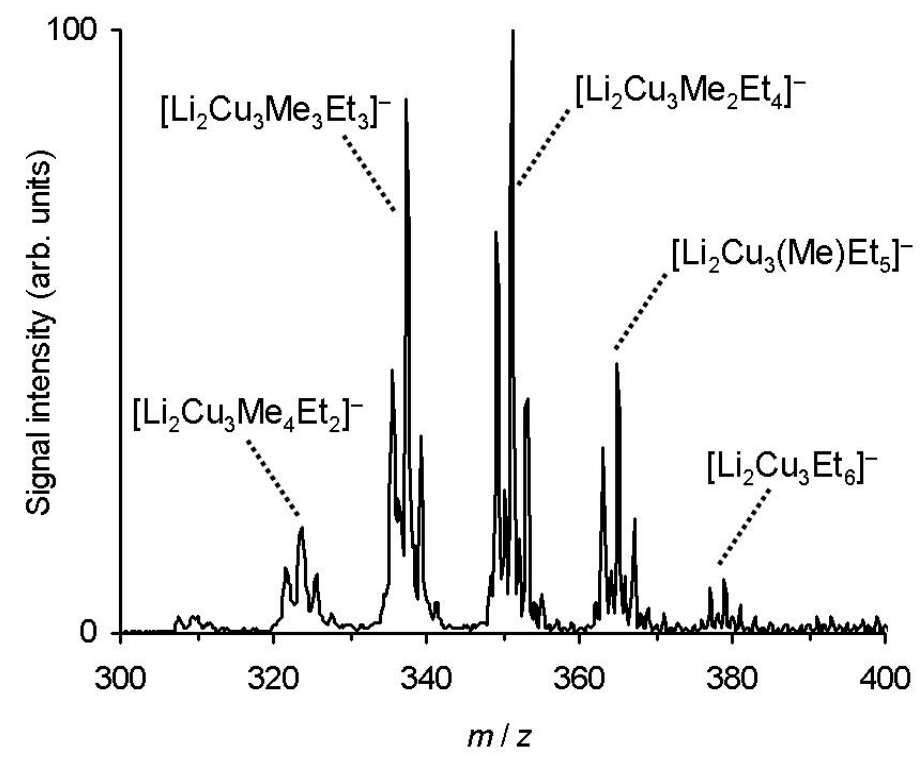

Figure S22. Section from the anion-mode ESI mass spectrum of a 1:1 mixture of $25 \mathrm{mM}$ solutions of $\mathrm{LiCuMe}_{2} \cdot \mathrm{LiCN}$ and $\mathrm{LiCuEt}_{2} \cdot \mathrm{LiCN}$ in THF.

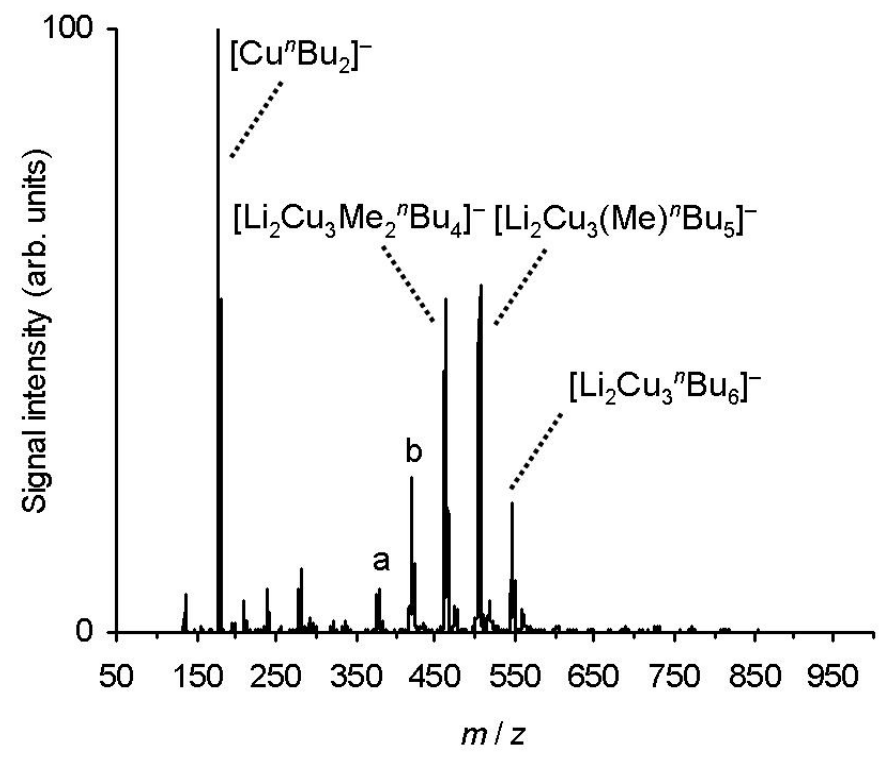

Figure S23. Anion-mode ESI mass spectrum of a 1:1 mixture of $25 \mathrm{mM}$ solutions of $\mathrm{LiCuMe}_{2} \cdot \mathrm{LiCN}$ and $\mathrm{LiCu}^{n} \mathrm{Bu}_{2} \cdot \mathrm{LiCN}$ in THF, $\mathrm{a}=\mathrm{Li}_{2} \mathrm{Cu}_{3} \mathrm{Me}_{4}{ }^{n} \mathrm{Bu}_{2}{ }^{-}, \mathrm{b}=\mathrm{Li}_{2} \mathrm{Cu}_{3} \mathrm{Me}_{3}{ }^{n} \mathrm{Bu}_{3}{ }^{-}$. 


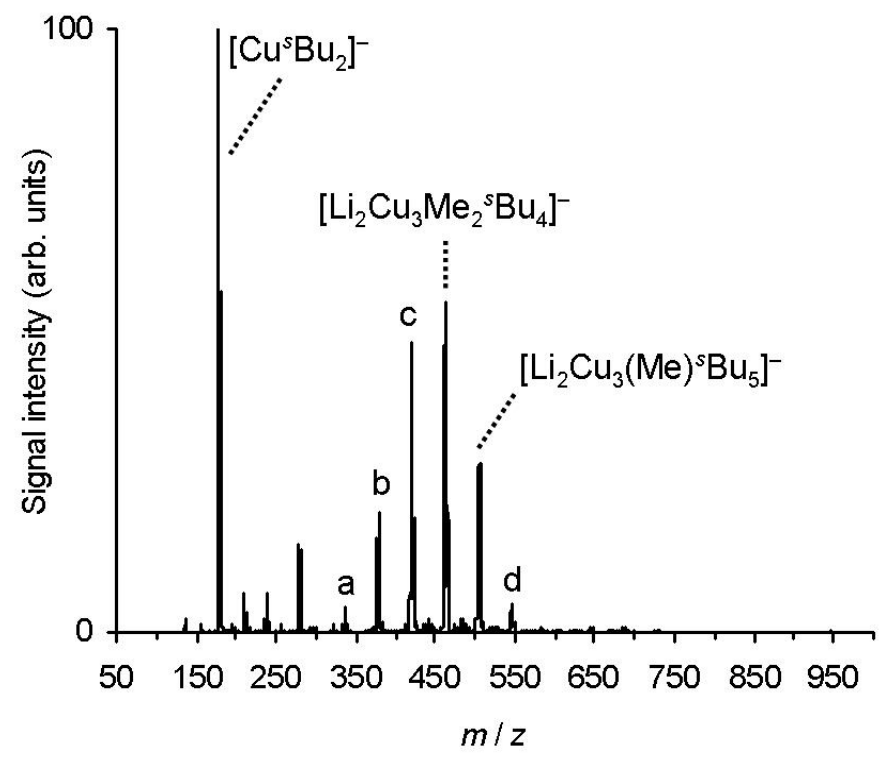

Figure S24. Anion-mode ESI mass spectrum of a 1:1 mixture of $25 \mathrm{mM}$ solutions of $\mathrm{LiCuMe}_{2} \cdot \mathrm{LiCN}$ and $\mathrm{LiCu}^{s} \mathrm{Bu}_{2} \cdot \mathrm{LiCN}$ in THF, $\mathrm{a}=\mathrm{Li}_{2} \mathrm{Cu}_{3} \mathrm{Me}_{5}{ }^{s} \mathrm{Bu}^{-}, \mathrm{b}=\mathrm{Li}_{2} \mathrm{Cu}_{3} \mathrm{Me}_{4}{ }^{s} \mathrm{Bu}_{2}{ }^{-}, \mathrm{c}=$ $\mathrm{Li}_{2} \mathrm{Cu}_{3} \mathrm{Me}_{3}{ }^{s} \mathrm{Bu}_{3}{ }^{-}, \mathrm{d}=\mathrm{Li}_{2} \mathrm{Cu}_{3}{ }^{s} \mathrm{Bu}_{6}{ }^{-}$.

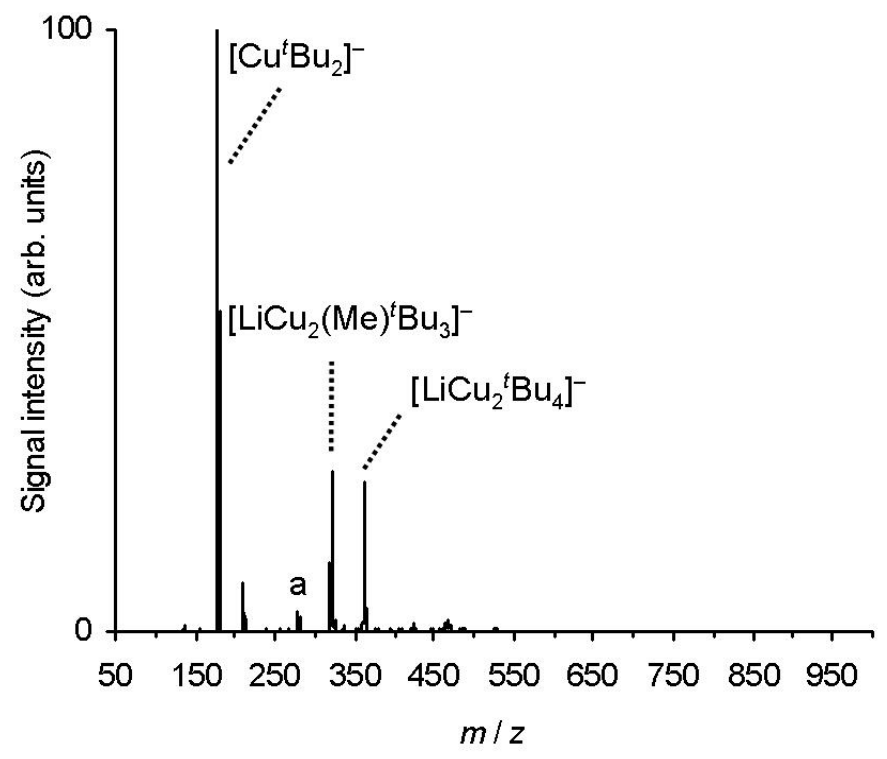

Figure S25. Anion-mode ESI mass spectrum of a 1:1 mixture of $25 \mathrm{mM}$ solutions of $\mathrm{LiCuMe}_{2} \cdot \mathrm{LiCN}$ and $\mathrm{LiCu}{ }^{t} \mathrm{Bu}_{2} \cdot \mathrm{LiCN}$ in THF, $\mathrm{a}=\mathrm{LiCu}_{2} \mathrm{Me}_{2}{ }^{t} \mathrm{Bu}_{2}{ }^{-}$. 


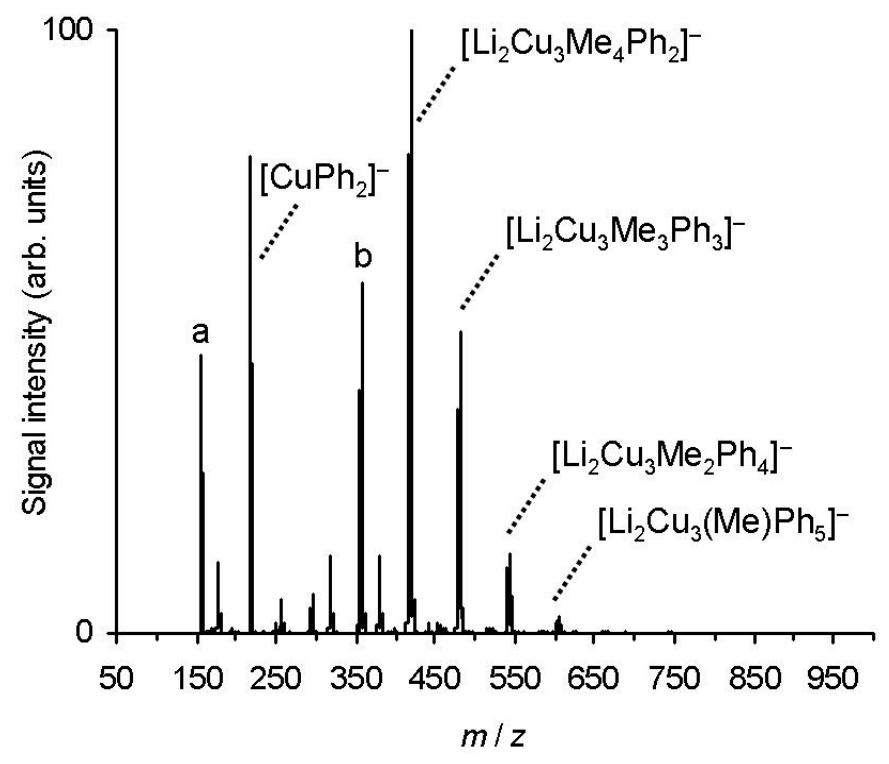

Figure S26. Anion-mode ESI mass spectrum of a 1:1 mixture of $25 \mathrm{mM}$ solutions of $\mathrm{LiCuMe}_{2} \cdot \mathrm{LiCN}$ and $\mathrm{LiCuPh}_{2} \cdot \mathrm{LiCN}$ in THF, $\mathrm{a}=\mathrm{Cu}(\mathrm{Me}) \mathrm{Ph}^{-}, \mathrm{b}=\mathrm{Li}_{2} \mathrm{Cu}_{3} \mathrm{Me}_{5} \mathrm{Ph}^{-}$. 


\section{6.) Additional anion-mode ESI mass spectra of $\operatorname{LiCuR(CN)}$ solutions}

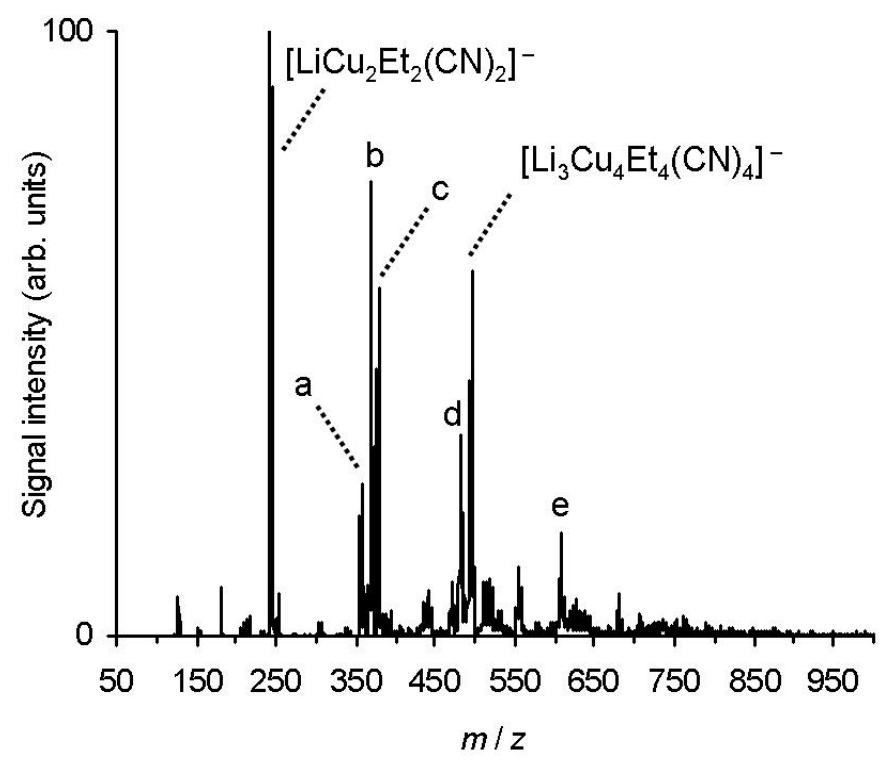

Figure S27. Anion-mode ESI mass spectrum of a $25 \mathrm{mM}$ solution of $\operatorname{LiCuEt}(\mathrm{CN})$ in $\mathrm{THF}, \mathrm{a}=$ $\mathrm{Cu}_{3} \mathrm{Et}_{2} \mathrm{Bu}(\mathrm{CN})_{2}^{-}, \quad \mathrm{b}=\mathrm{Li}_{2} \mathrm{Cu}_{3} \mathrm{Et}_{3}(\mathrm{CN})_{3}{ }^{-}, \quad \mathrm{c}=\mathrm{Li}_{2} \mathrm{Cu}_{3} \mathrm{Et}_{6}^{-}, \mathrm{d}=\mathrm{LiCu}_{4} \mathrm{Et}_{3} \mathrm{Bu}(\mathrm{CN})_{3}{ }^{-}, \quad \mathrm{e}=$ $\mathrm{Li}_{2} \mathrm{Cu}_{5} \mathrm{Et} \mathrm{Bu}_{4} \mathrm{Bu}(\mathrm{CN})_{4}^{-}$. The presence of $\mathrm{Cu}(+2)$-containing species with butyl substituents is inferred from fragmentation experiments. The butyl moieties possibly originate from carbometalation of ethene, which supposedly results from $\beta$-hydrogen elimination of ethyllithium or -copper species. 


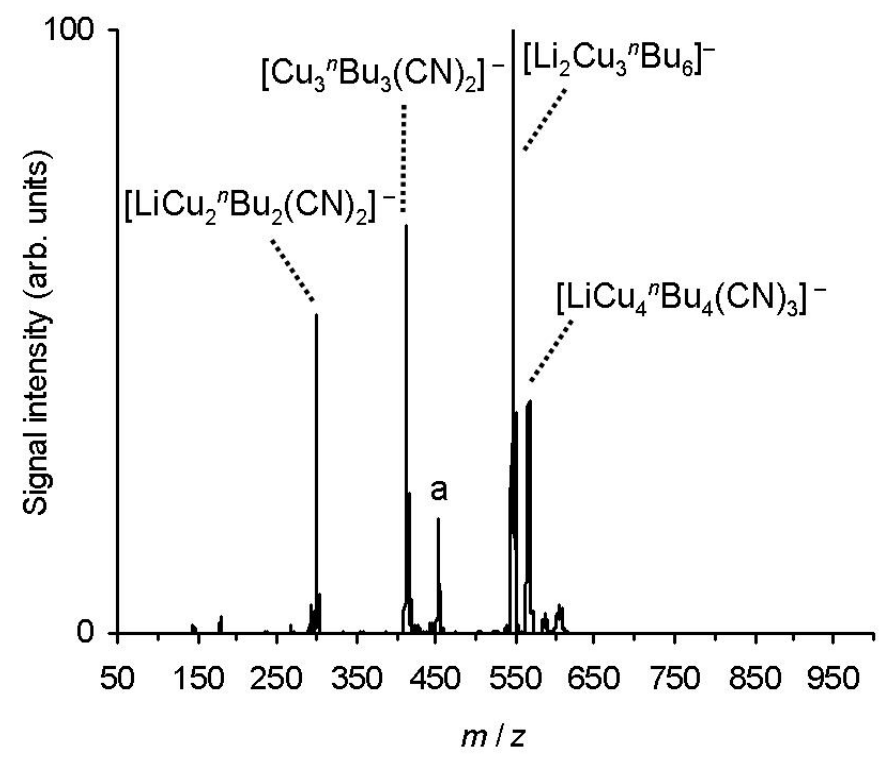

Figure S28. Anion-mode ESI mass spectrum of a $25 \mathrm{mM}$ solution of $\mathrm{LiCu}^{n} \mathrm{Bu}(\mathrm{CN})$ in THF, a $=$ $\mathrm{Li}_{2} \mathrm{Cu}_{3}{ }^{n} \mathrm{Bu}_{3}(\mathrm{CN})_{3}^{-}$.

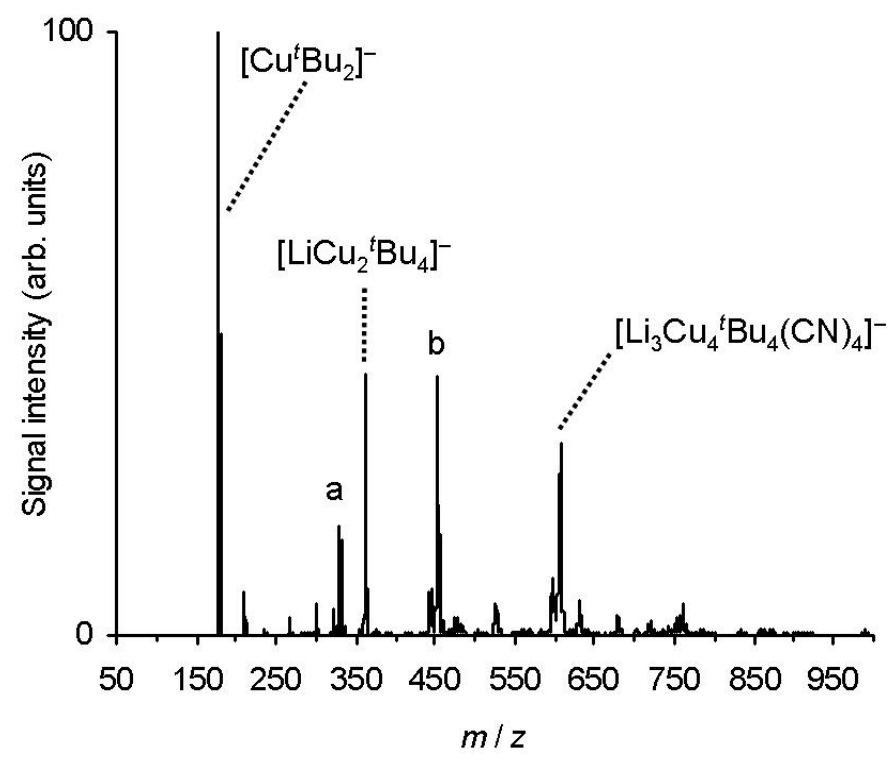

Figure S29. Anion-mode ESI mass spectrum of a $25 \mathrm{mM}$ solution of $\mathrm{LiCu}^{t} \mathrm{Bu}(\mathrm{CN})$ in $\mathrm{THF}, \mathrm{a}=$ $\mathrm{LiCu}_{2}{ }^{t} \mathrm{Bu}_{3}(\mathrm{CN})^{-}, \mathrm{b}=\mathrm{Li}_{2} \mathrm{Cu}_{3}{ }^{t} \mathrm{Bu}_{3}(\mathrm{CN})_{3}{ }^{-}$. 


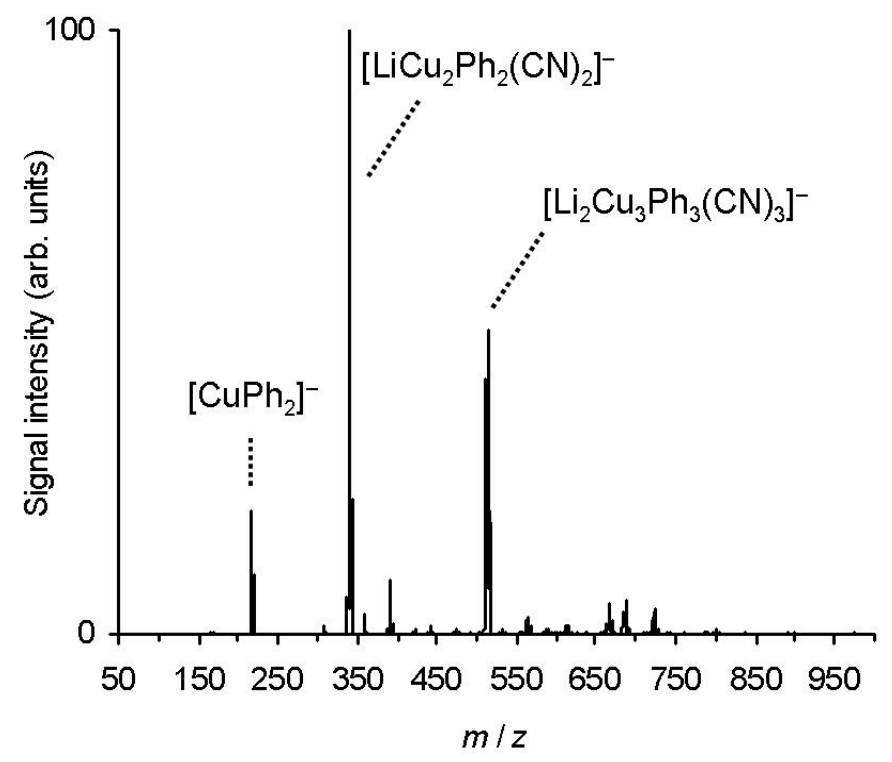

Figure S30. Anion-mode ESI mass spectrum of a $25 \mathrm{mM}$ solution of $\mathrm{LiCuPh}(\mathrm{CN})$ in $\mathrm{THF}$.

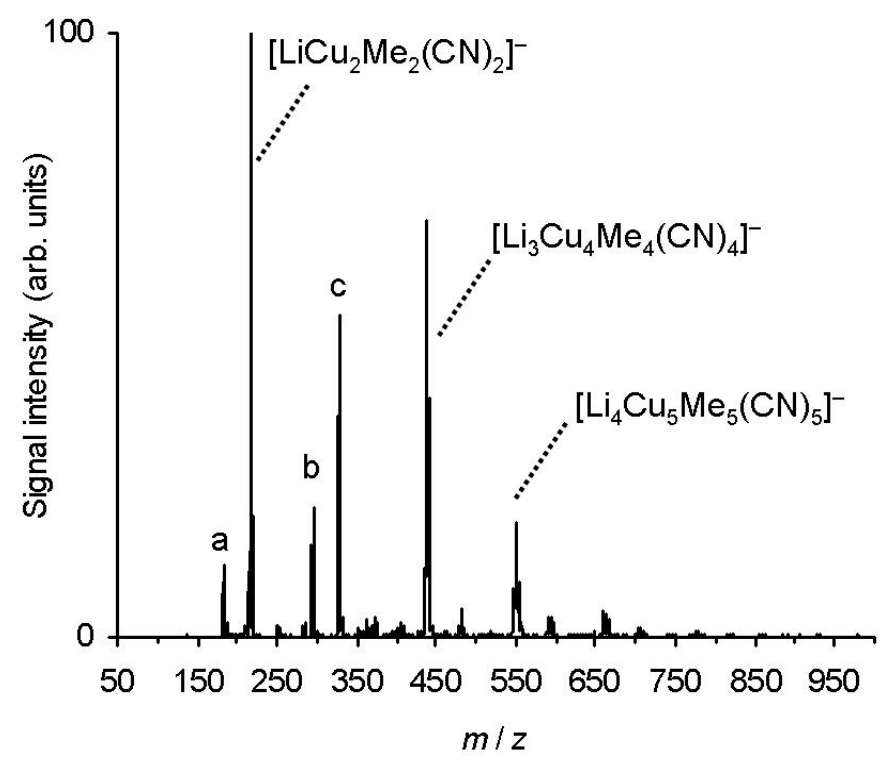

Figure S31. Anion-mode ESI mass spectrum of a $25 \mathrm{mM}$ solution of $\mathrm{Li}_{0.8} \mathrm{CuMe}_{0.8}(\mathrm{CN})$ in $\mathrm{THF}$, a $=\mathrm{Cu}_{2} \mathrm{Me}_{2}(\mathrm{CN})^{-}, \mathrm{b}=\mathrm{Li}_{2} \mathrm{Cu}_{3} \mathrm{Me}_{6}^{-}, \mathrm{c}=\mathrm{Li}_{2} \mathrm{Cu}_{3} \mathrm{Me}_{3}(\mathrm{CN})_{3}{ }^{-}$. 


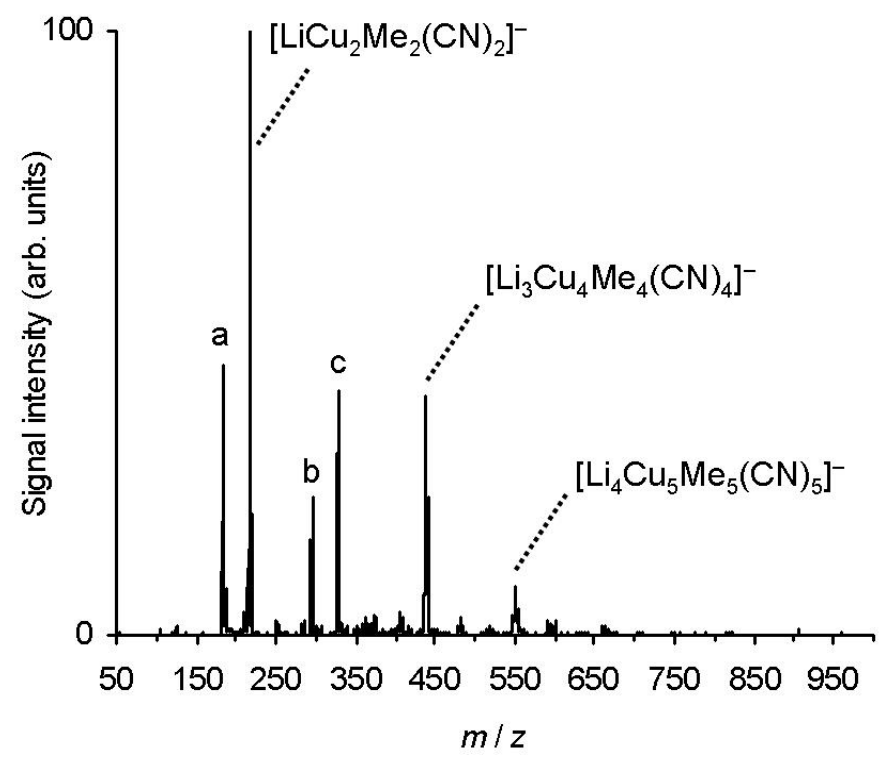

Figure S32. Anion-mode ESI mass spectrum of a $25 \mathrm{mM}$ solution of $\operatorname{Li}_{0.5} \mathrm{CuMe}_{0.5}(\mathrm{CN})$ in $\mathrm{THF}$, a $=\mathrm{Cu}_{2} \mathrm{Me}_{2}(\mathrm{CN})^{-}, \mathrm{b}=\mathrm{Li}_{2} \mathrm{Cu}_{3} \mathrm{Me}_{6}^{-}, \mathrm{c}=\mathrm{Li}_{2} \mathrm{Cu}_{3} \mathrm{Me}_{3}(\mathrm{CN})_{3}{ }^{-}$.

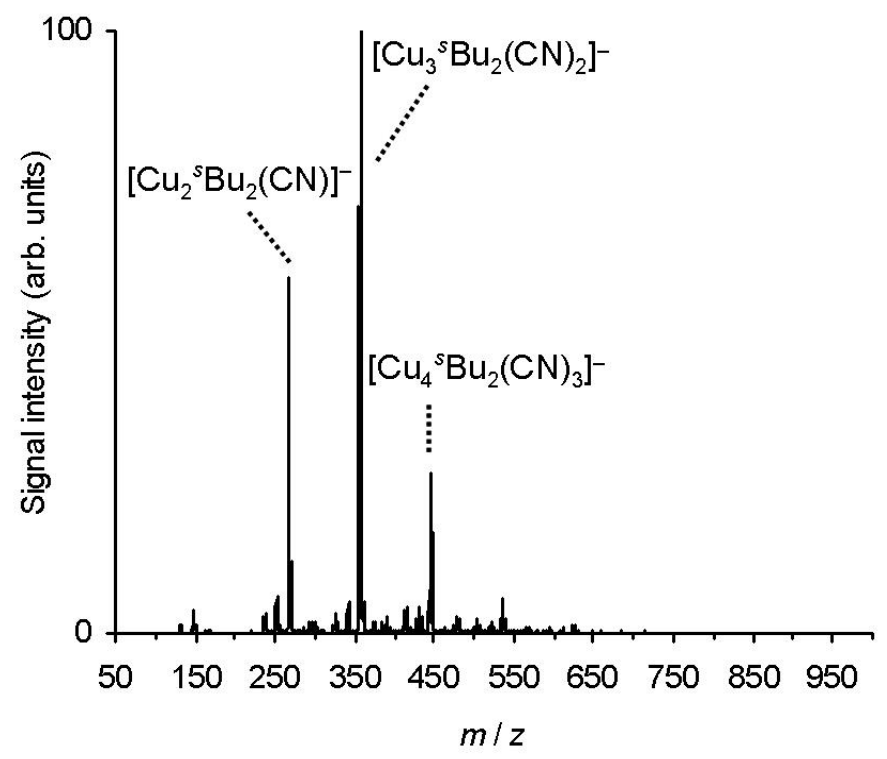

Figure S33. Anion-mode ESI mass spectrum of a $25 \mathrm{mM}$ solution of $\mathrm{LiCu}^{s} \mathrm{Bu}_{2} \cdot \mathrm{LiCN}$ in $\mathrm{THF}$ after brief exposure to air. 


\section{7.) Additional cation-mode ESI mass spectra of $\mathrm{LiCuR}_{2} \cdot \mathrm{LiCN}$ solutions}

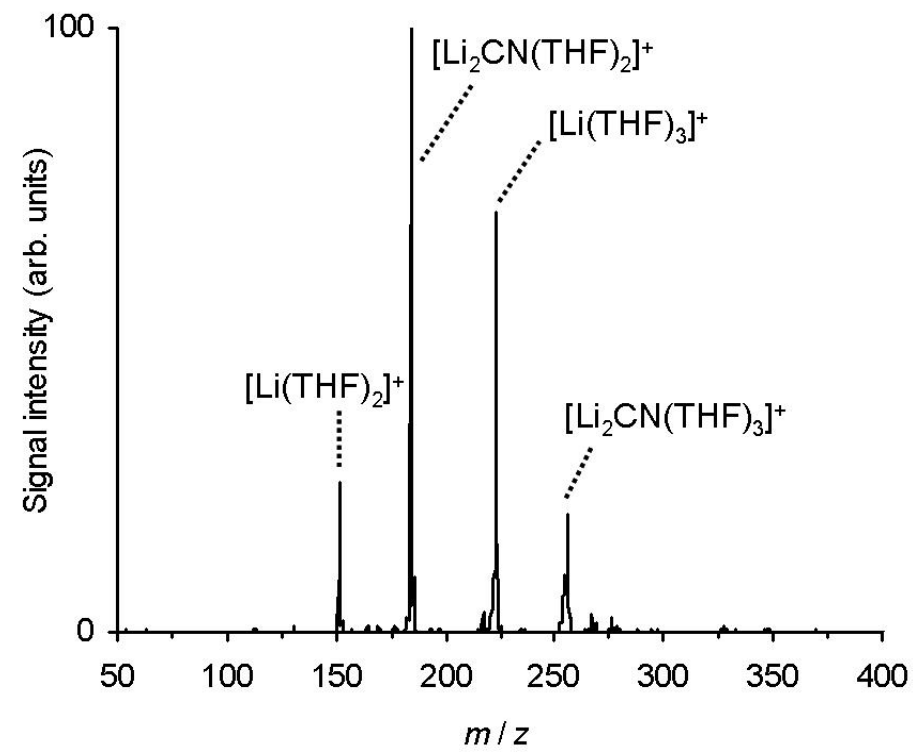

Figure S34. Cation-mode ESI mass spectrum of a $25 \mathrm{mM}$ solution of $\mathrm{LiCuEt}_{2} \cdot \mathrm{LiCN}$ in THF.

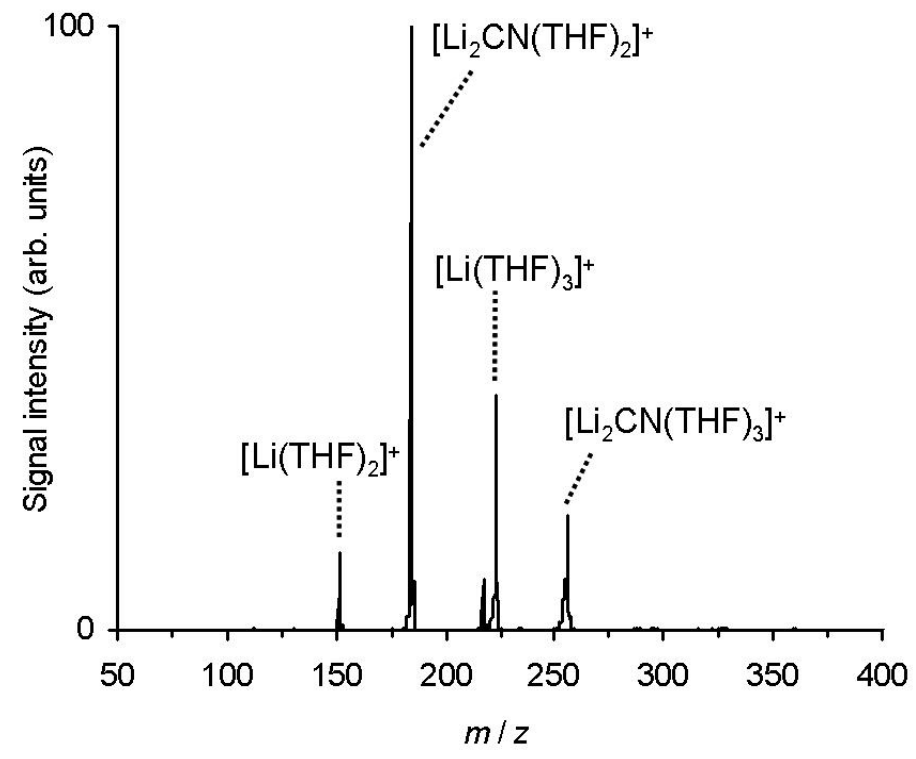

Figure S35. Cation-mode ESI mass spectrum of a $25 \mathrm{mM}$ solution of $\mathrm{LiCu}^{n} \mathrm{Bu}_{2} \cdot \mathrm{LiCN}$ in THF. 


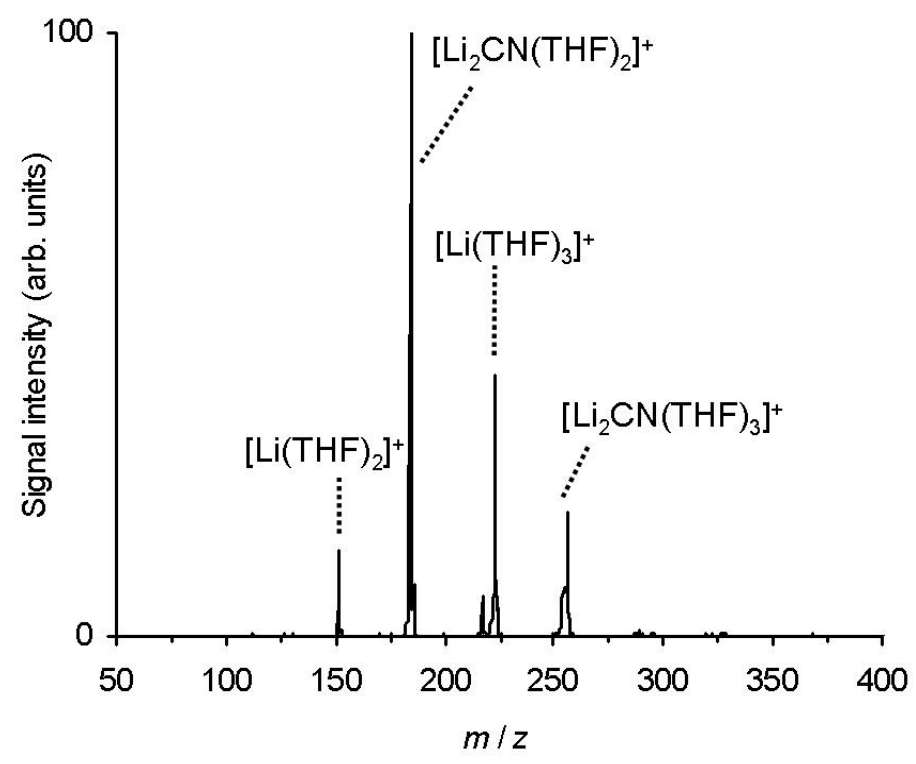

Figure S36. Cation-mode ESI mass spectrum of a $25 \mathrm{mM}$ solution of $\mathrm{LiCu}^{s} \mathrm{Bu}_{2} \cdot \mathrm{LiCN}$ in THF.

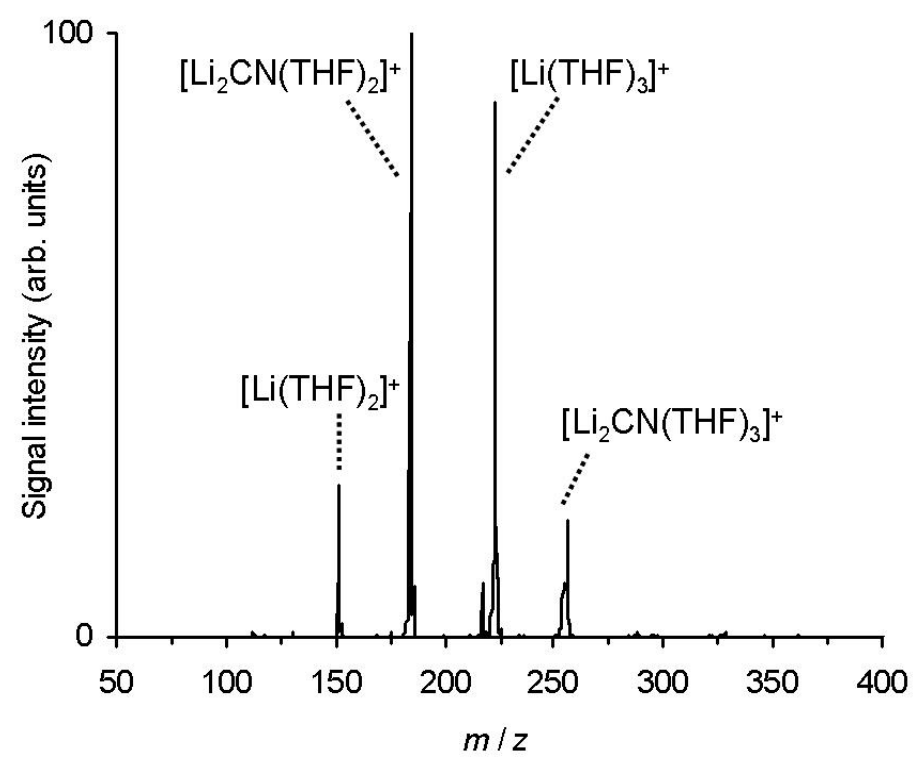

Figure S37. Cation-mode ESI mass spectrum of a $25 \mathrm{mM}$ solution of $\mathrm{LiCu}^{t} \mathrm{Bu}_{2} \cdot \mathrm{LiCN}$ in THF. 


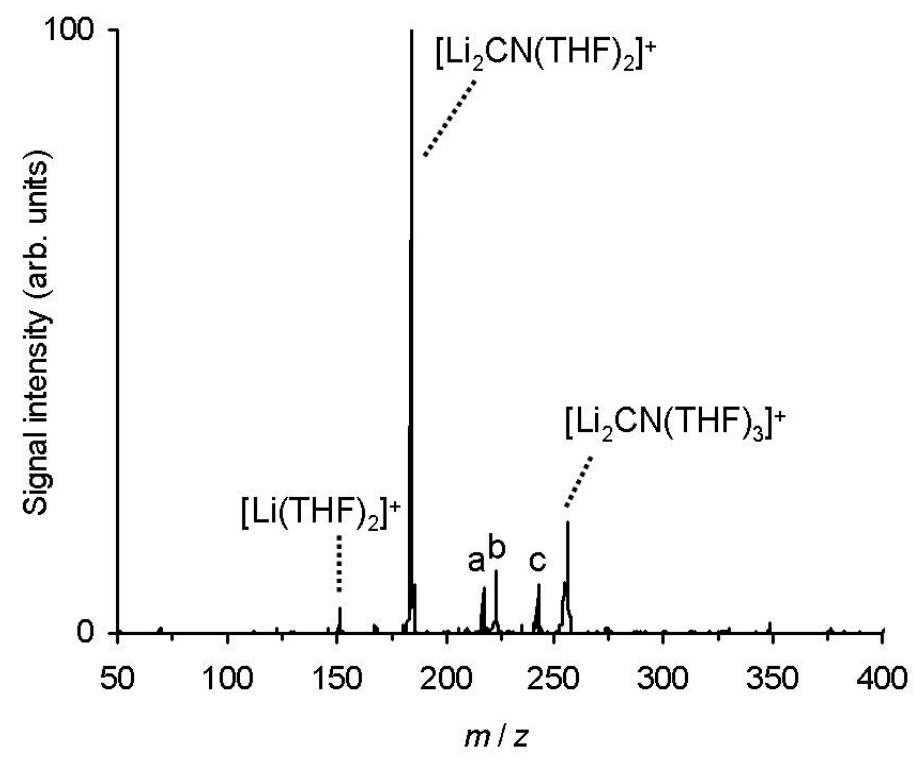

Figure S38. Cation-mode ESI mass spectrum of a $25 \mathrm{mM}$ solution of $\mathrm{LiCuPh}_{2} \cdot \mathrm{LiCN}$ in THF, a $=$ $\mathrm{Li}_{3}(\mathrm{CN})_{2}(\mathrm{THF})_{2}{ }^{+}, \mathrm{b}=\mathrm{Li}(\mathrm{THF})_{3}{ }^{+}, \mathrm{c}=\mathrm{Li}_{2} \mathrm{CN}(\mathrm{THF})\left({ }^{n} \mathrm{Bu}_{2} \mathrm{O}\right){ }^{+} .{ }^{n} \mathrm{Bu}_{2} \mathrm{O}$ stems from the PhLi solutions used for the preparation of $\mathrm{LiCuPh}_{2} \cdot \mathrm{LiCN}$. 


\section{8.) Additional cation-mode ESI mass spectra of $\mathrm{Li}_{0.8} \mathrm{CuR}_{0.8}(\mathrm{CN})$ solutions}

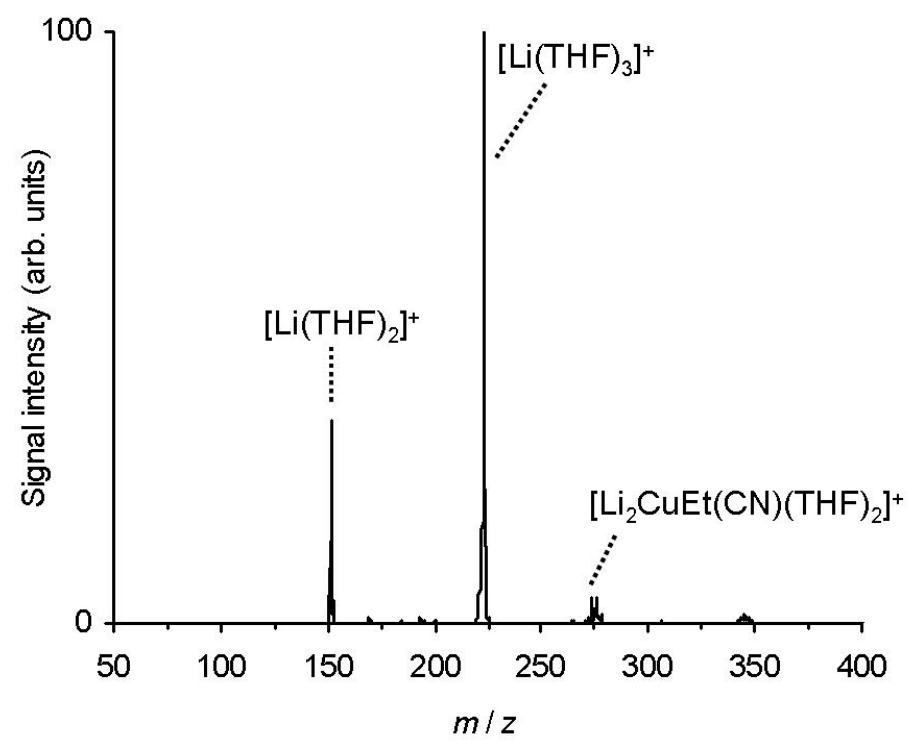

Figure S39. Cation-mode ESI mass spectrum of a $25 \mathrm{mM}$ solution of $\mathrm{Li}_{0.8} \mathrm{CuEt}_{0.8}(\mathrm{CN})$ in THF.

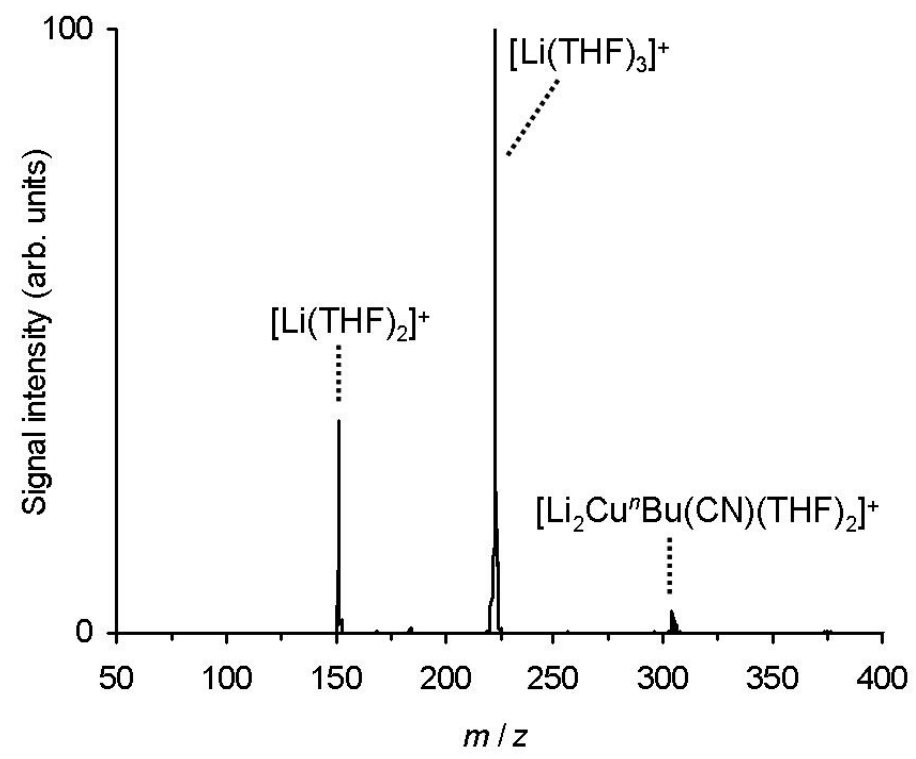

Figure S40. Cation-mode ESI mass spectrum of a $25 \mathrm{mM}$ solution of $\mathrm{Li}_{0.8} \mathrm{Cu}^{n} \mathrm{Bu}_{0.8}(\mathrm{CN})$ in THF. 


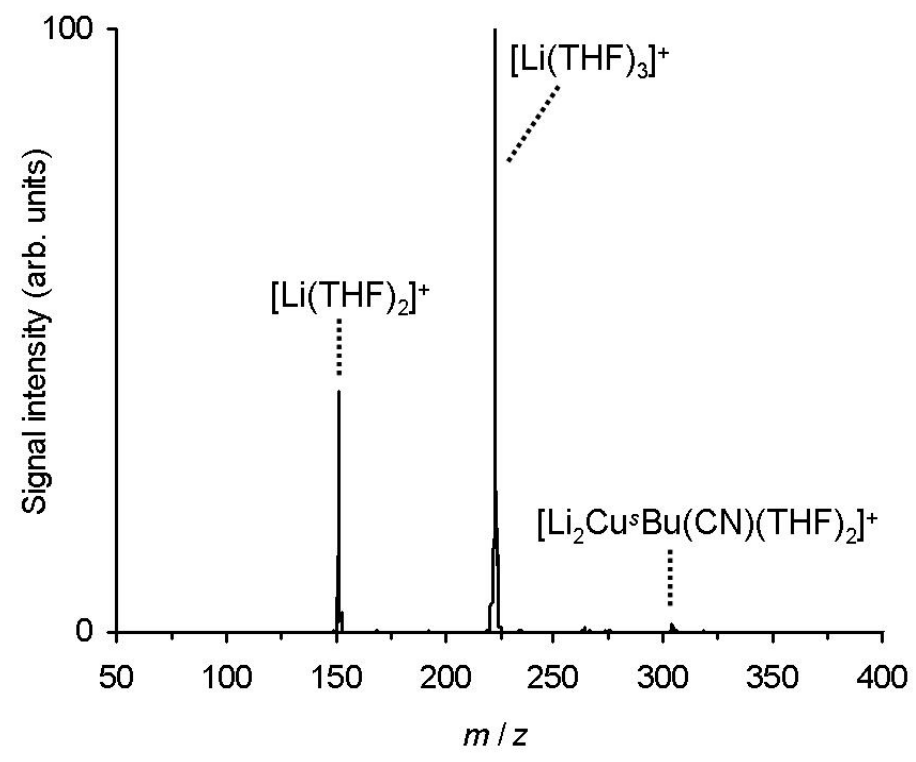

Figure S41. Cation-mode ESI mass spectrum of a $25 \mathrm{mM}$ solution of $\mathrm{Li}_{0.8} \mathrm{Cu}^{s} \mathrm{Bu}_{0.8}(\mathrm{CN})$ in $\mathrm{THF}$.

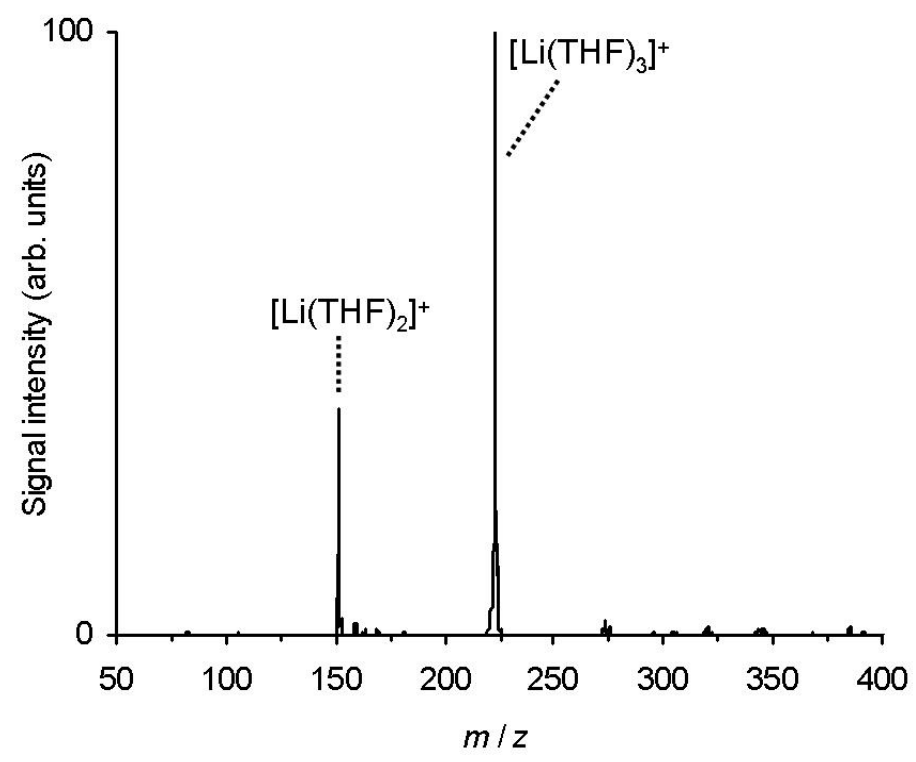

Figure S42. Cation-mode ESI mass spectrum of a $25 \mathrm{mM}$ solution of $\mathrm{Li}_{0.8} \mathrm{Cu}^{t} \mathrm{Bu}_{0.8}(\mathrm{CN})$ in $\mathrm{THF}$. 


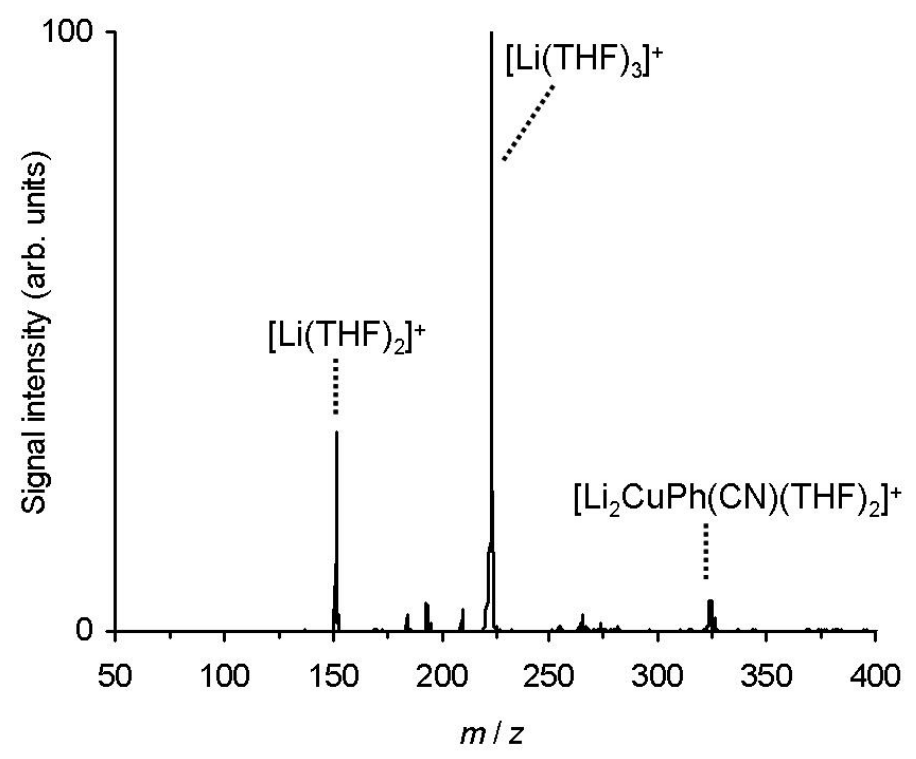

Figure S43. Cation-mode ESI mass spectrum of a $25 \mathrm{mM}$ solution of $\mathrm{Li}_{0.8} \mathrm{CuPh}_{0.8}(\mathrm{CN})$ in $\mathrm{THF}$.

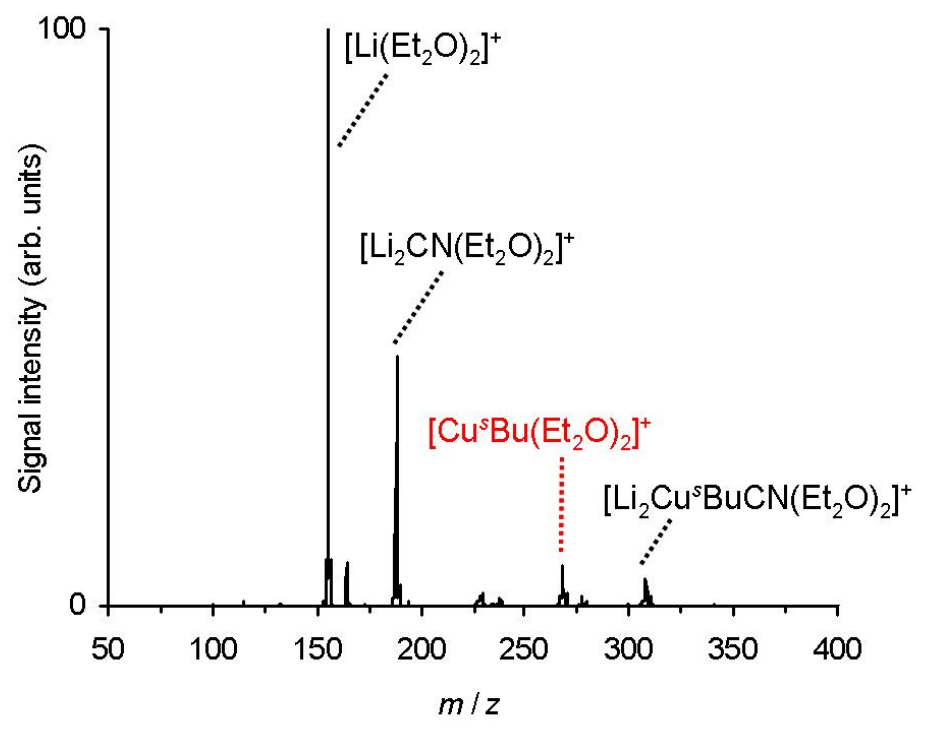

Figure S44. Cation-mode ESI mass spectrum of a $25 \mathrm{mM}$ solution of $\mathrm{Li}_{0.8} \mathrm{Cu}^{s} \mathrm{Bu}_{0.8}(\mathrm{CN})$ in $\mathrm{Et}_{2} \mathrm{O}$. The $\mathrm{Cu}(+2)$-containing ion is shown in red. 


\section{9.) Mass spectra of mass-selected $\mathrm{Li}_{n-1} \mathrm{Cu}_{n} \mathrm{R}_{2 n}{ }^{-}$anions and their fragment ions}

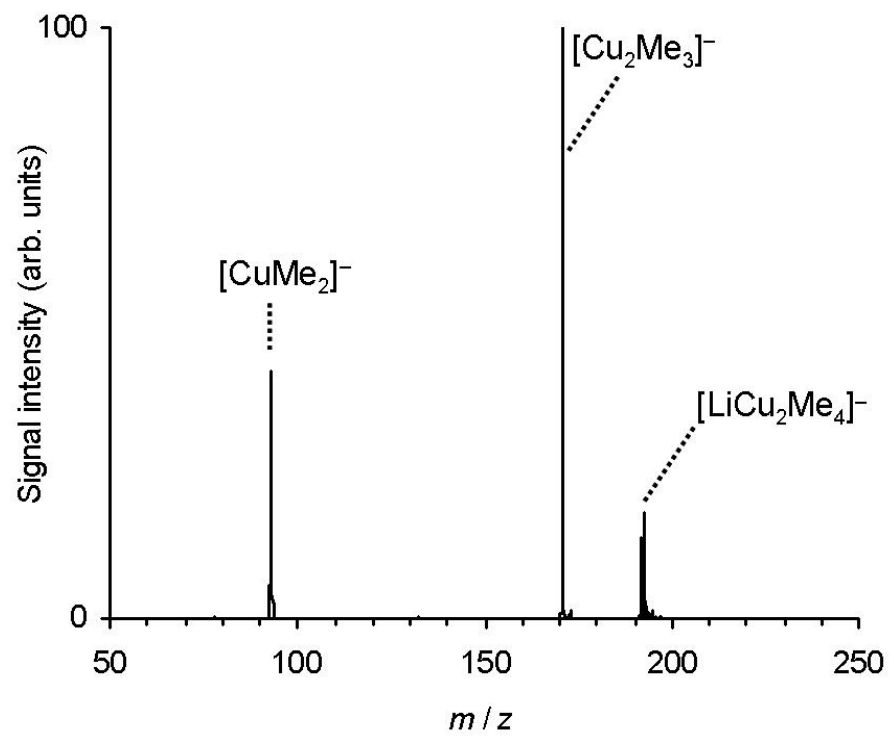

Figure S45. Mass spectrum of mass-selected $\mathrm{LiCu}_{2} \mathrm{Me}_{4}{ }^{-}(m / z=193)$ and its fragment ions produced upon collision-induced dissociation $\left(V_{\mathrm{exc}}=0.30 \mathrm{~V}\right)$.

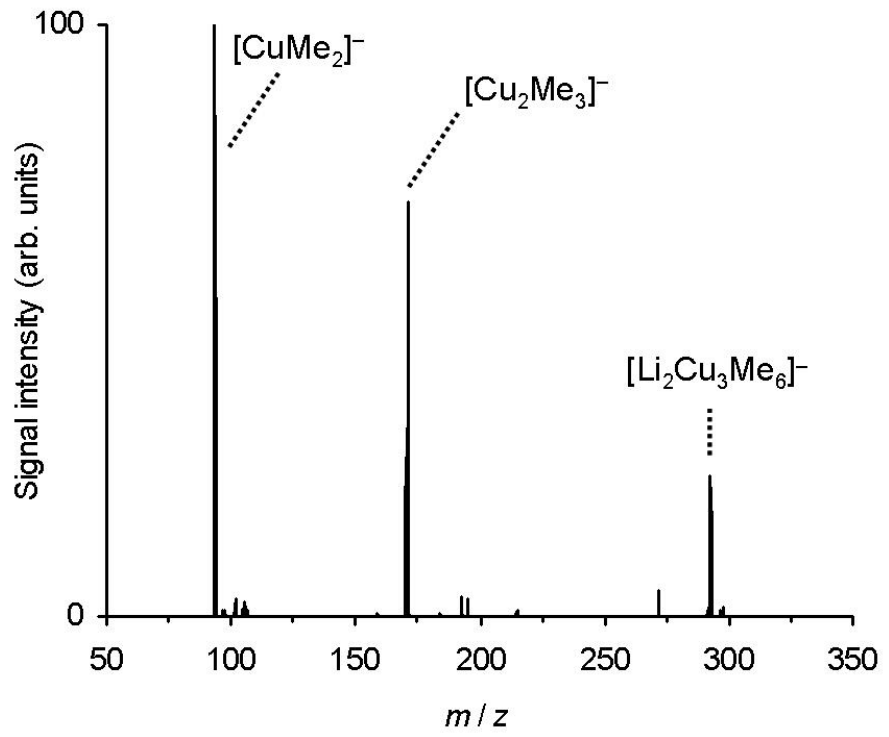

Figure S46. Mass spectrum of mass-selected $\mathrm{Li}_{2} \mathrm{Cu}_{3} \mathrm{Me}_{6}{ }^{-}(m / z=293)$ and its fragment ions produced upon collision-induced dissociation $\left(V_{\mathrm{exc}}=0.30 \mathrm{~V}\right)$. 


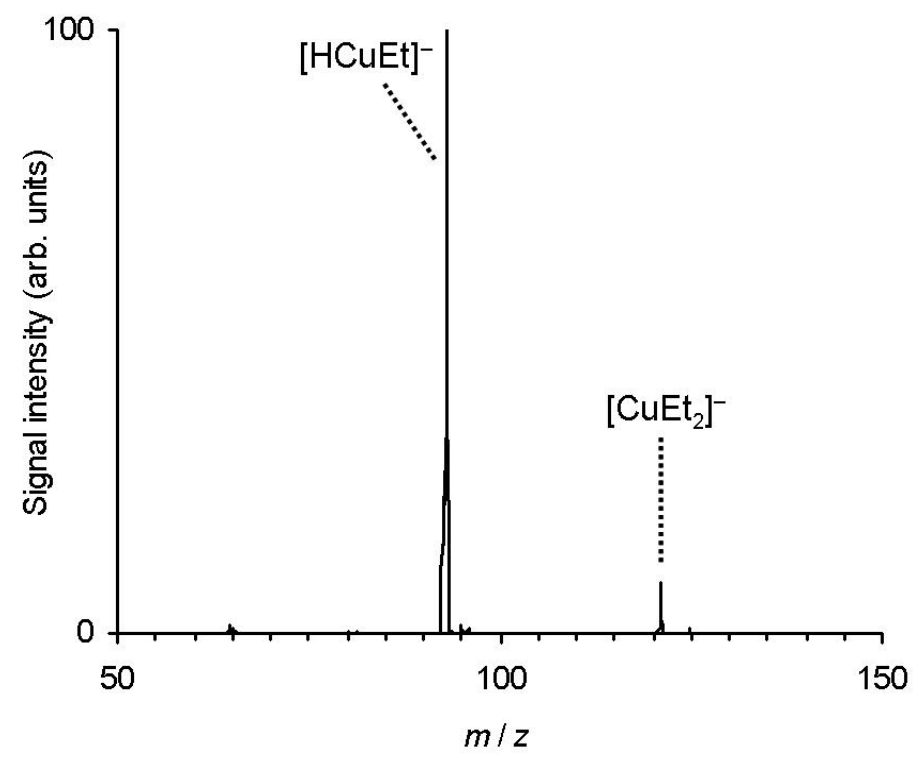

Figure S47. Mass spectrum of mass-selected $\mathrm{CuEt}_{2}{ }^{-}(\mathrm{m} / \mathrm{z}=121)$ and its fragment ions produced upon collision-induced dissociation $\left(V_{\mathrm{exc}}=0.35 \mathrm{~V}\right)$.

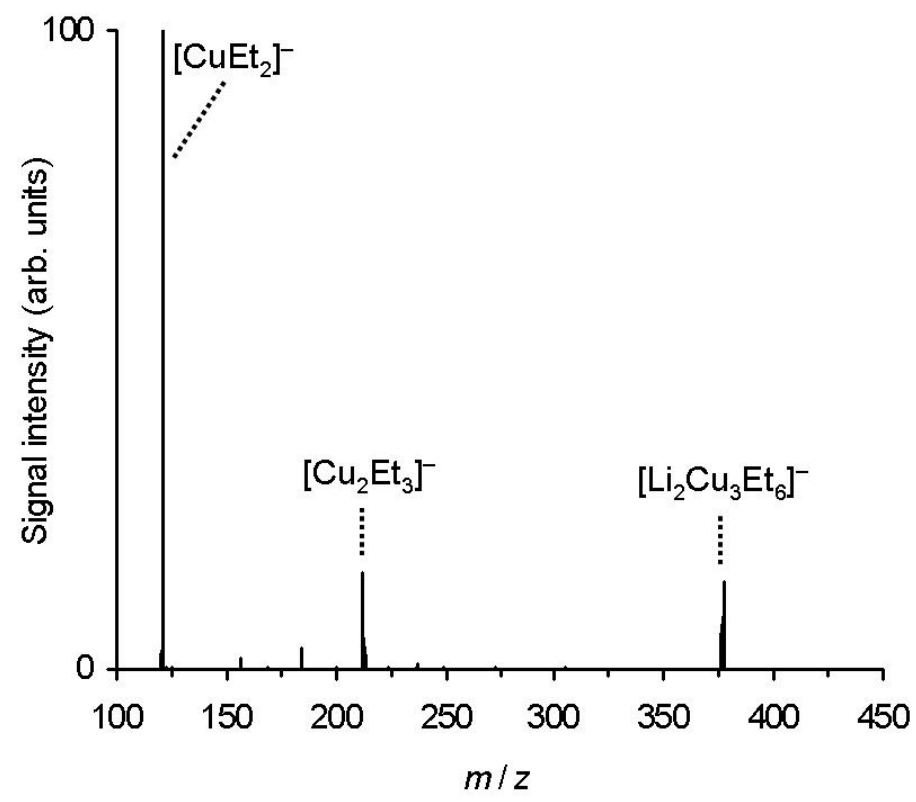

Figure S48. Mass spectrum of mass-selected $\mathrm{Li}_{2} \mathrm{Cu}_{3} \mathrm{Et}_{6}{ }^{-}(m / z=377)$ and its fragment ions produced upon collision-induced dissociation $\left(V_{\mathrm{exc}}=0.30 \mathrm{~V}\right)$. 


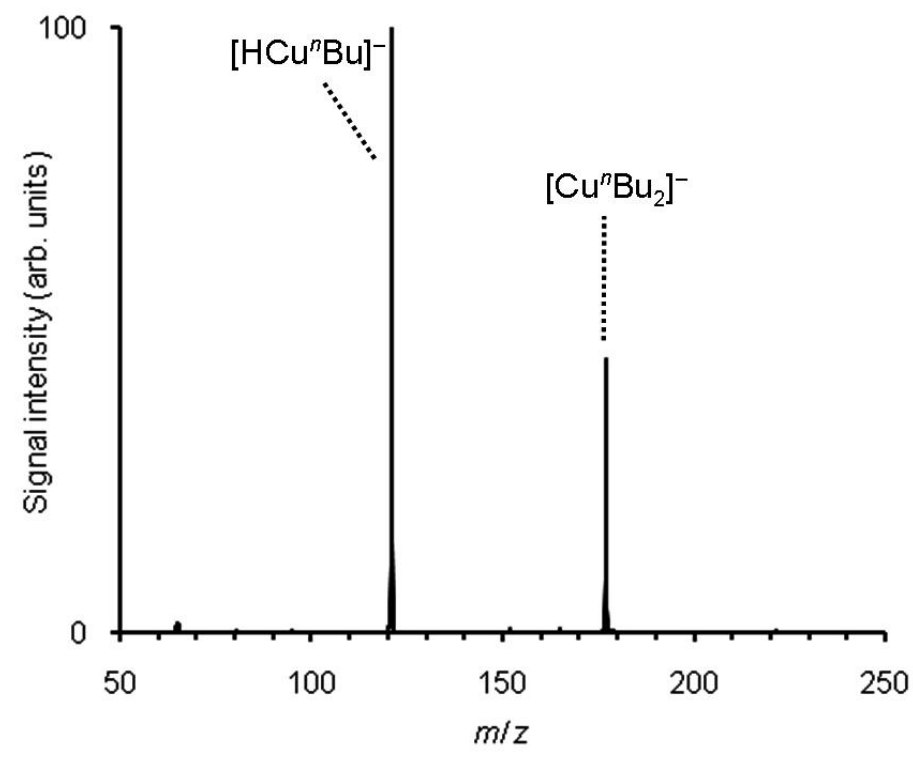

Figure S49. Mass spectrum of mass-selected $\mathrm{Cu}^{n} \mathrm{Bu}_{2}^{-}(m / z=177)$ and its fragment ions produced upon collision-induced dissociation $\left(V_{\mathrm{exc}}=0.25 \mathrm{~V}\right)$.

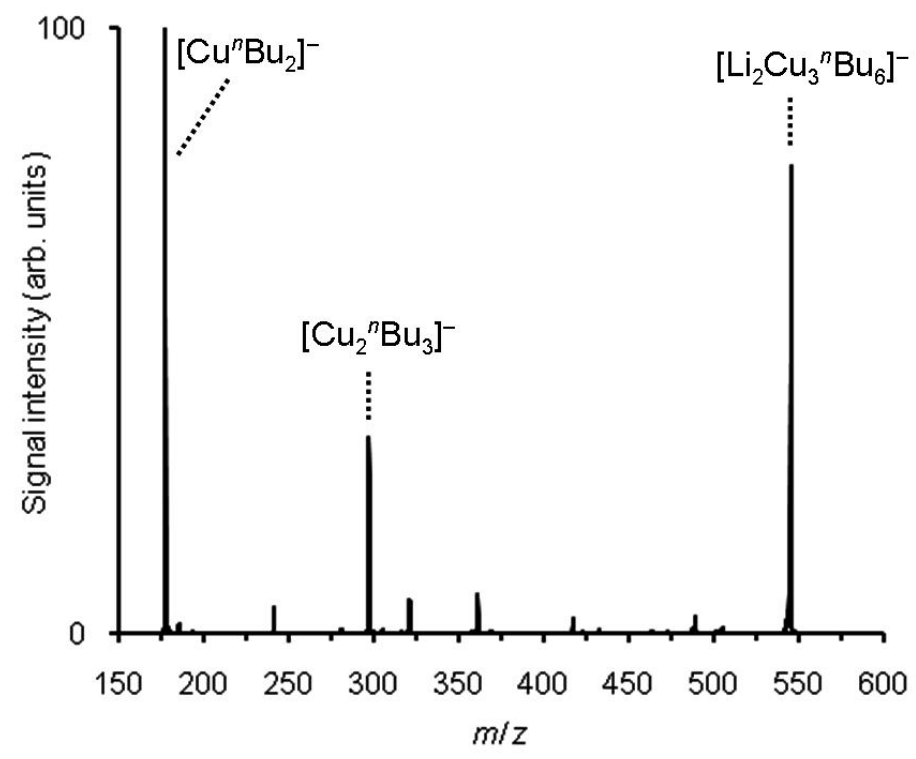

Figure S50. Mass spectrum of mass-selected $\mathrm{Li}_{2} \mathrm{Cu}_{3}{ }^{n} \mathrm{Bu}_{6}{ }^{-}(m / z=545)$ and its fragment ions produced upon collision-induced dissociation $\left(V_{\mathrm{exc}}=0.23 \mathrm{~V}\right)$. 


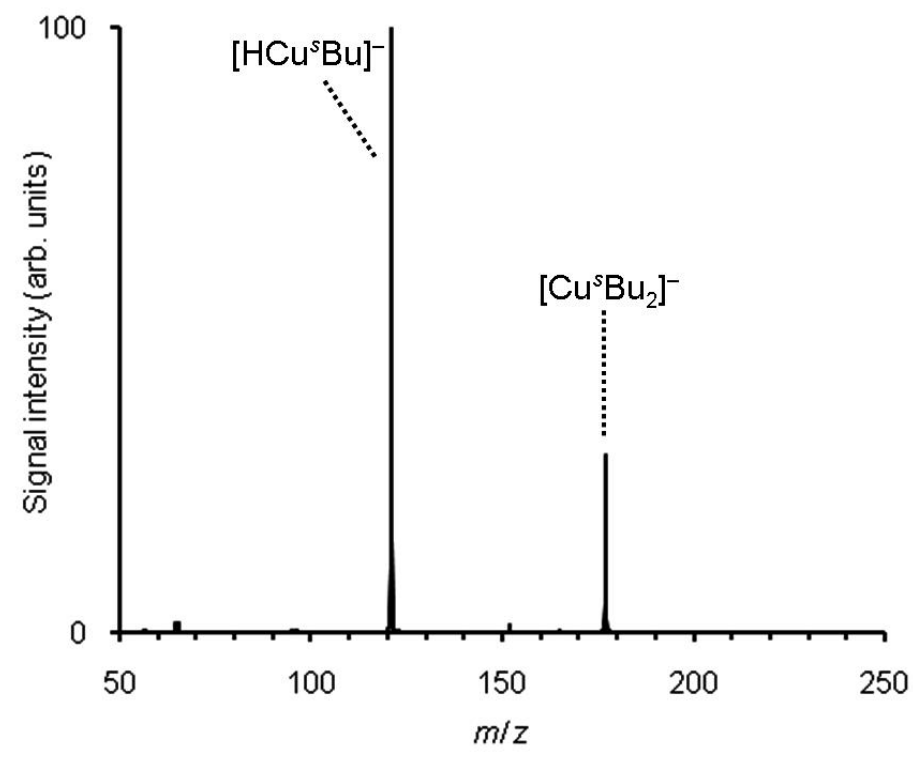

Figure S51. Mass spectrum of mass-selected $\mathrm{Cu}^{s} \mathrm{Bu}_{2}^{-}(m / z=177)$ and its fragment ions produced upon collision-induced dissociation $\left(V_{\mathrm{exc}}=0.25 \mathrm{~V}\right)$.

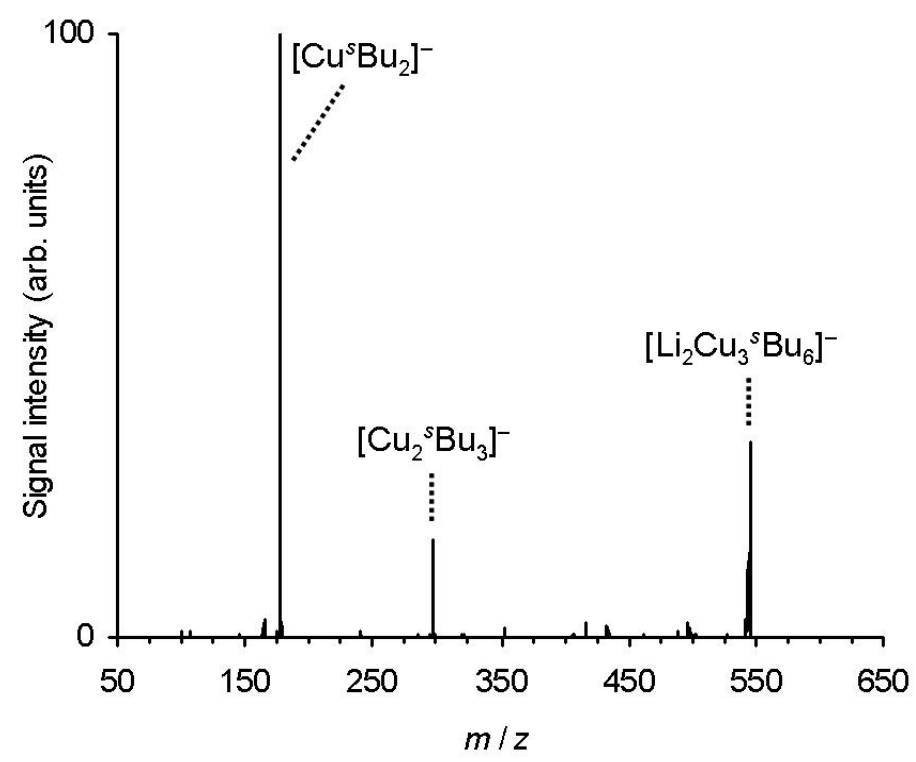

Figure S52. Mass spectrum of mass-selected $\mathrm{Li}_{2} \mathrm{Cu}_{3}{ }^{s} \mathrm{Bu}_{6}{ }^{-}(m / z=545)$ and its fragment ions produced upon collision-induced dissociation $\left(V_{\mathrm{exc}}=0.20 \mathrm{~V}\right)$. 


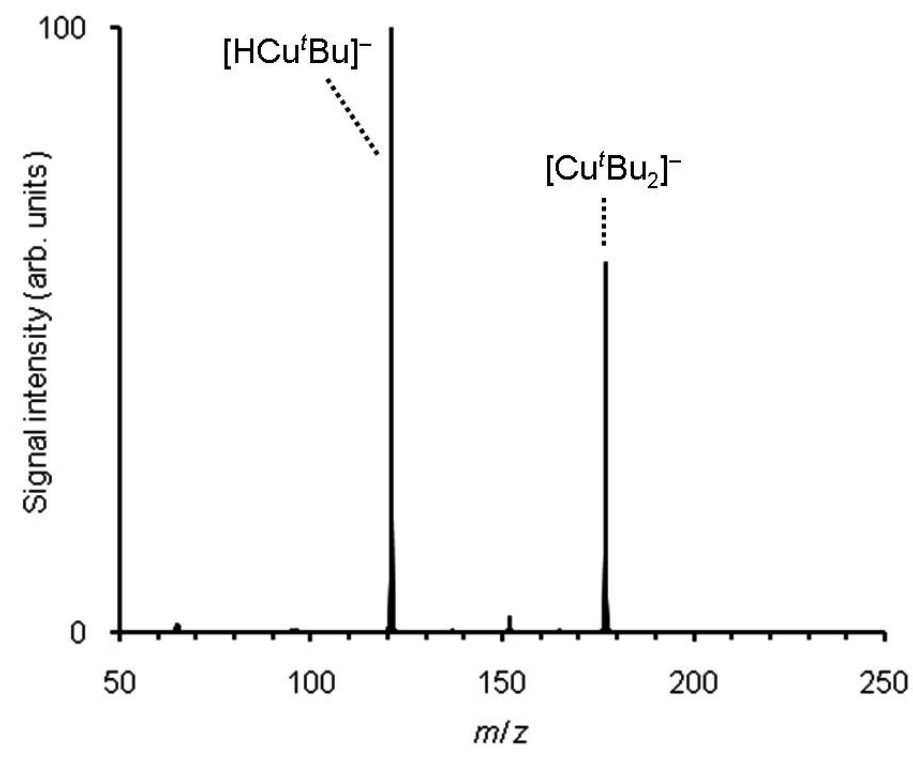

Figure S53. Mass spectrum of mass-selected $\mathrm{Cu}^{t} \mathrm{Bu}_{2}{ }^{-}(m / z=177)$ and its fragment ions produced upon collision-induced dissociation $\left(V_{\mathrm{exc}}=0.25 \mathrm{~V}\right)$.

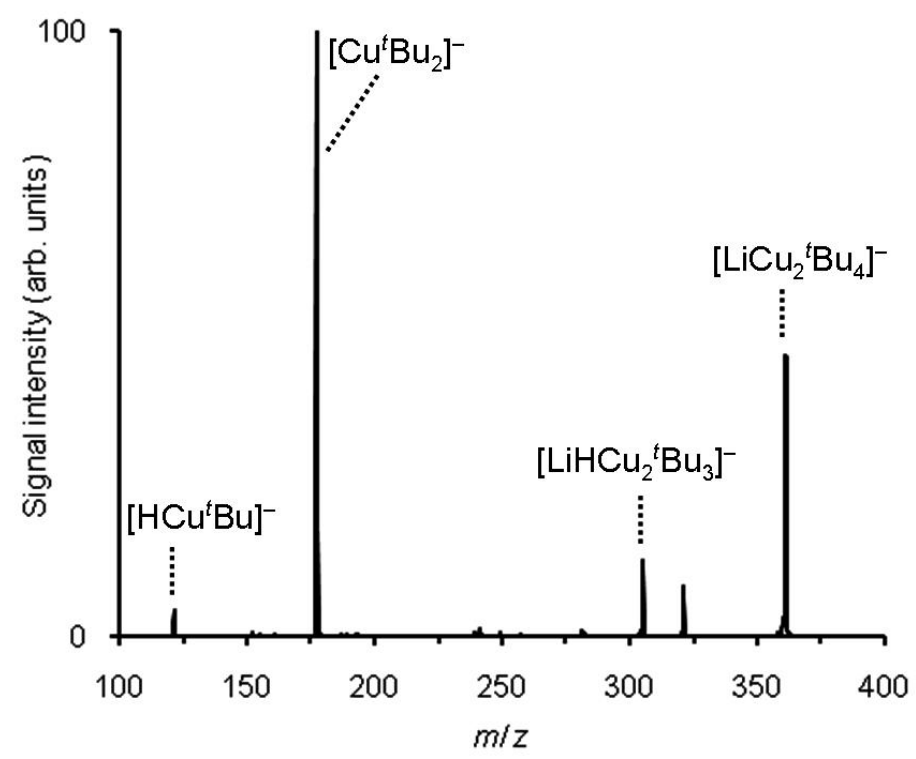

Figure S54. Mass spectrum of mass-selected $\mathrm{LiCu}_{2}{ }^{t} \mathrm{Bu}_{4}{ }^{-}(m / z=361)$ and its fragment ions produced upon collision-induced dissociation $\left(V_{\mathrm{exc}}=0.30 \mathrm{~V}\right)$. 


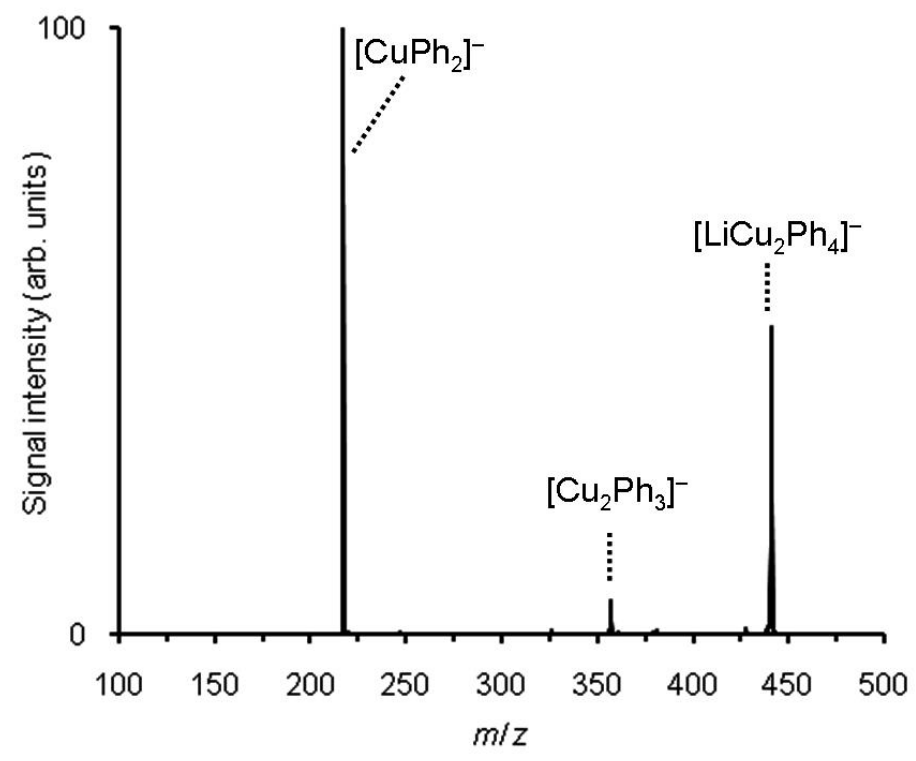

Figure S55. Mass spectrum of mass-selected $\mathrm{LiCu}_{2} \mathrm{Ph}_{4}^{-}(m / z=441)$ and its fragment ions produced upon collision-induced dissociation $\left(V_{\mathrm{exc}}=0.20 \mathrm{~V}\right)$.

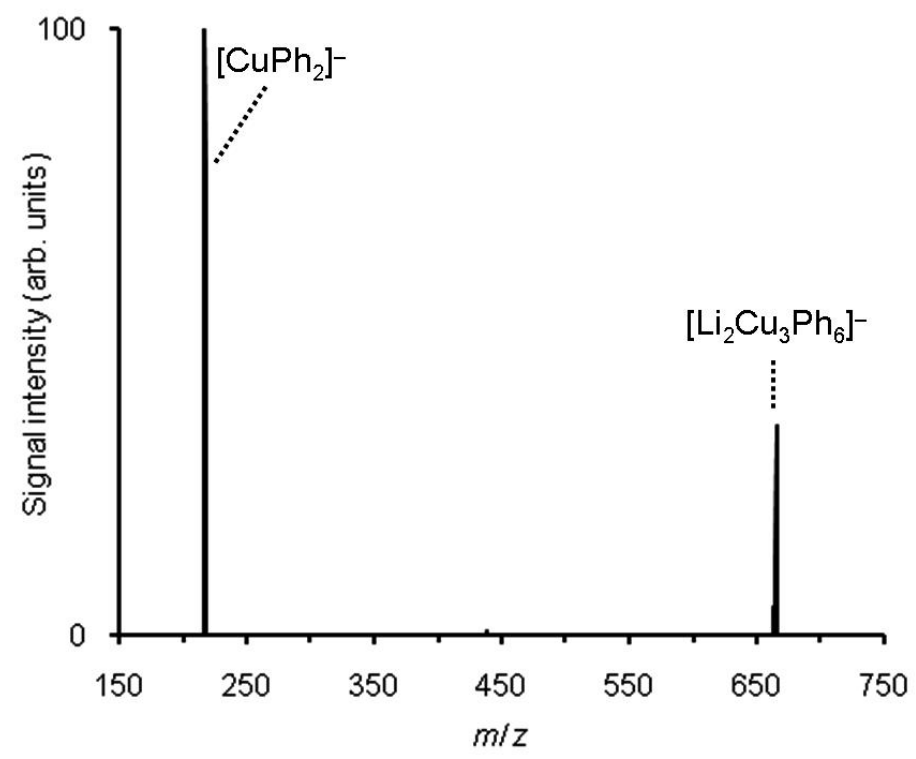

Figure S56. Mass spectrum of mass-selected $\mathrm{Li}_{2} \mathrm{Cu}_{3} \mathrm{Ph}_{6}{ }^{-}(m / z=665)$ and its fragment ions produced upon collision-induced dissociation $\left(V_{\mathrm{exc}}=0.20 \mathrm{~V}\right)$. 
10.) Mass spectra of mass-selected $\mathrm{Li}_{n-1} \mathrm{Cu}_{n} \mathrm{Me}_{2 n-x} \mathrm{R}_{x}{ }^{-}$anions and their fragment ions

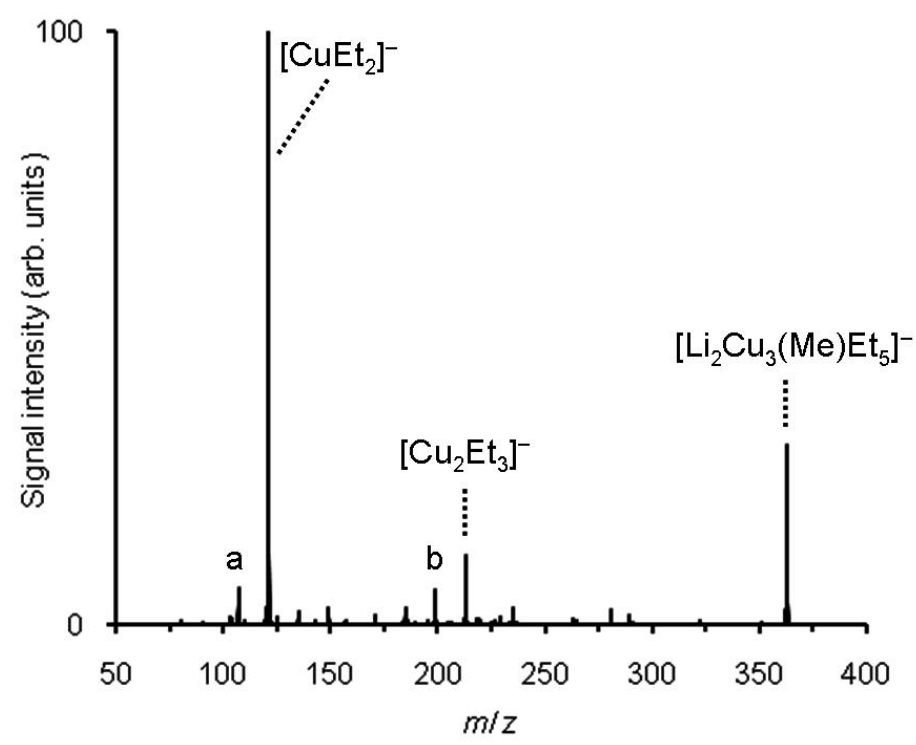

Figure S57. Mass spectrum of mass-selected $\mathrm{Li}_{2} \mathrm{Cu}_{3}(\mathrm{Me}) \mathrm{Et}_{5}{ }^{-}(\mathrm{m} / z=363)$ and its fragment ions produced upon collision-induced dissociation $\left(V_{\mathrm{exc}}=0.29 \mathrm{~V}\right), \mathrm{a}=\mathrm{Cu}(\mathrm{Me}) \mathrm{Et}^{-}, \mathrm{b}=\mathrm{Cu}_{2}(\mathrm{Me}) \mathrm{Et}_{2}{ }^{-}$.

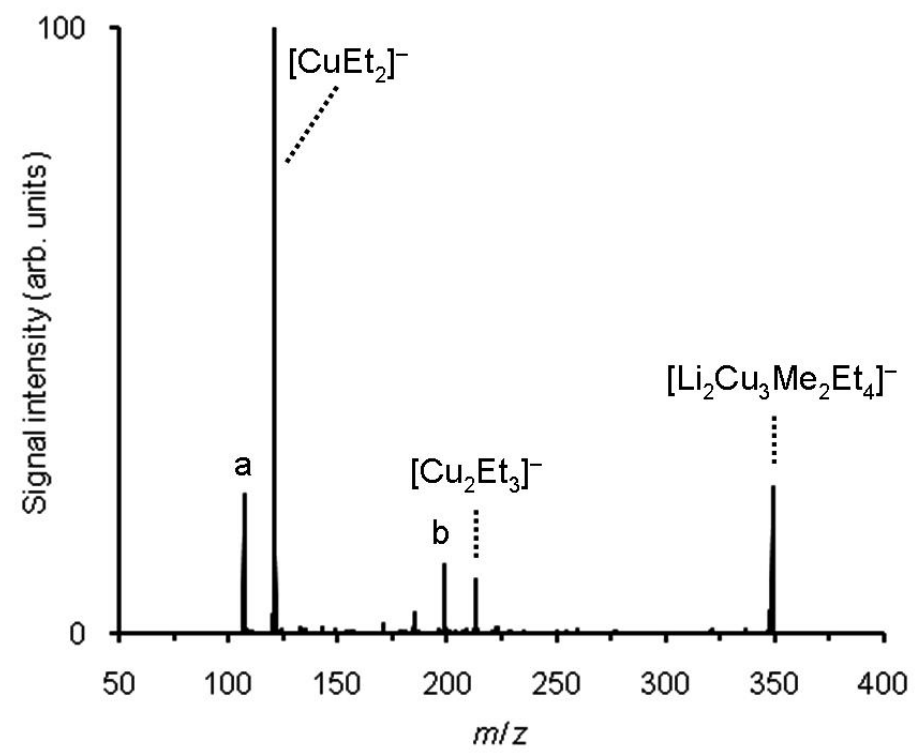

Figure S58. Mass spectrum of mass-selected $\mathrm{Li}_{2} \mathrm{Cu}_{3} \mathrm{Me}_{2} \mathrm{Et}_{4}{ }^{-}(m / z=349)$ and its fragment ions produced upon collision-induced dissociation $\left(V_{\mathrm{exc}}=0.29 \mathrm{~V}\right), \mathrm{a}=\mathrm{Cu}(\mathrm{Me}) \mathrm{Et}^{-}, \mathrm{b}=\mathrm{Cu}_{2}(\mathrm{Me}) \mathrm{Et}_{2}{ }^{-}$. 


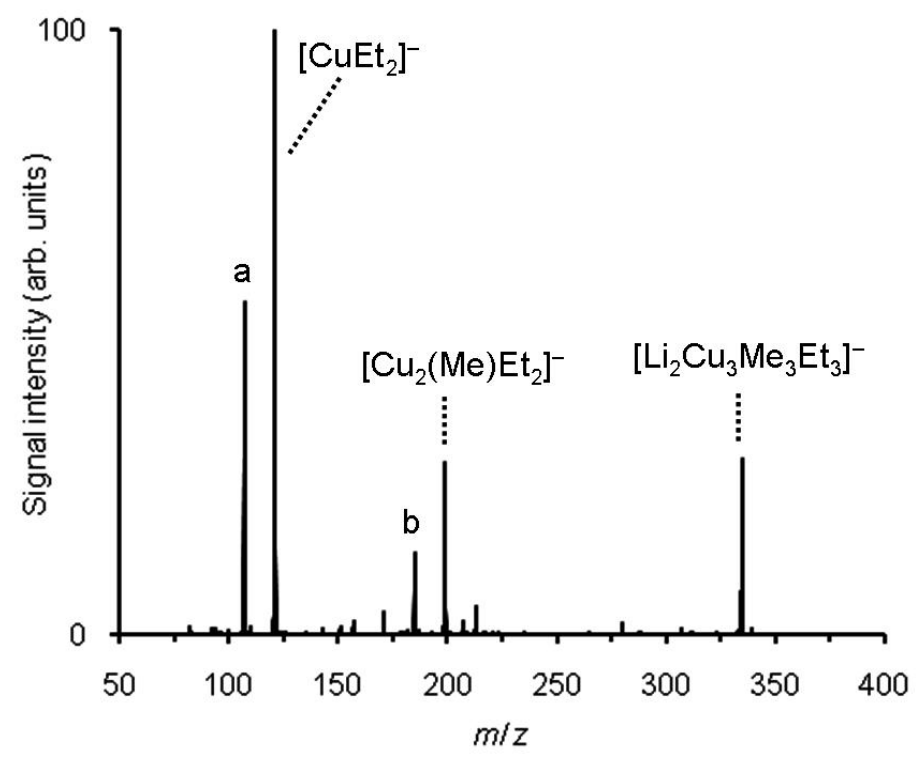

Figure S59. Mass spectrum of mass-selected $\mathrm{Li}_{2} \mathrm{Cu}_{3} \mathrm{Me}_{3} \mathrm{Et}_{3}{ }^{-}(m / z=335)$ and its fragment ions produced upon collision-induced dissociation $\left(V_{\mathrm{exc}}=0.29 \mathrm{~V}\right), \mathrm{a}=\mathrm{Cu}(\mathrm{Me}) \mathrm{Et}^{-}, \mathrm{b}=\mathrm{Cu}_{2} \mathrm{Me}_{2} \mathrm{Et}^{-}$.

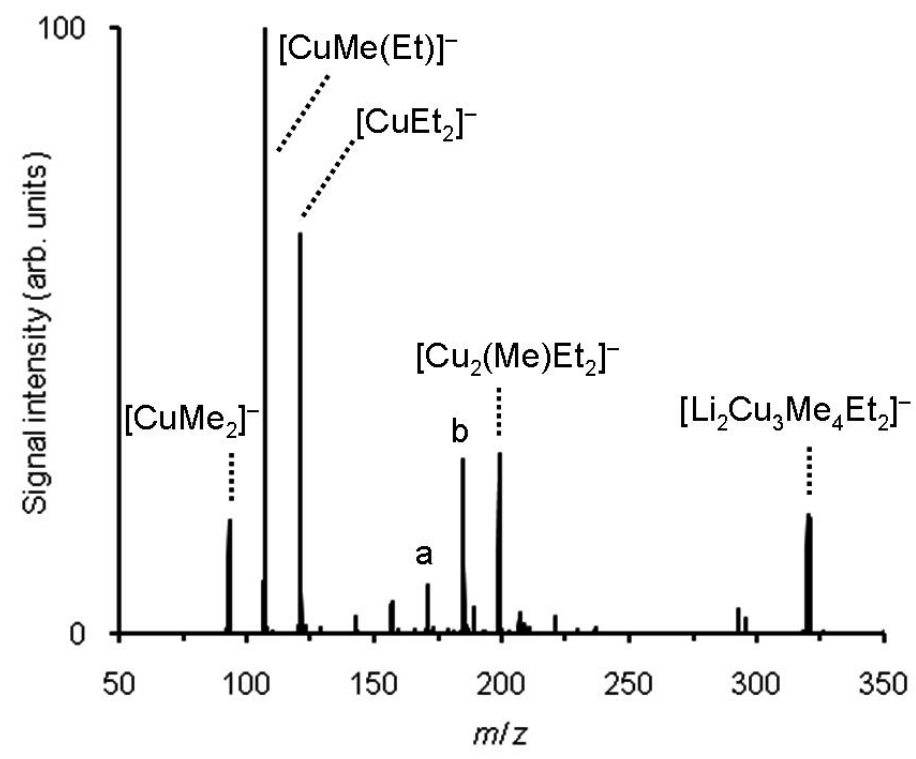

Figure S60. Mass spectrum of mass-selected $\mathrm{Li}_{2} \mathrm{Cu}_{3} \mathrm{Me}_{4} \mathrm{Et}_{2}{ }^{-}(\mathrm{m} / z=321)$ and its fragment ions produced upon collision-induced dissociation $\left(V_{\mathrm{exc}}=0.29 \mathrm{~V}\right), \mathrm{a}=\mathrm{Cu}_{2} \mathrm{Me}_{3}{ }^{-}, \mathrm{b}=\mathrm{Cu}_{2} \mathrm{Me}_{2} \mathrm{Et}^{-}$. 


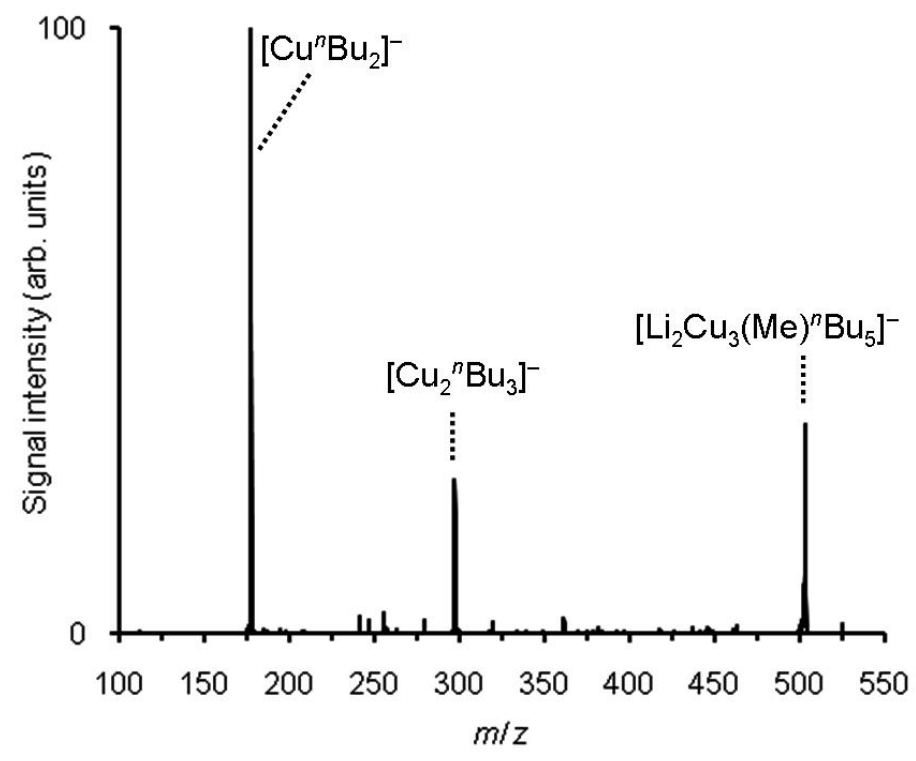

Figure S61. Mass spectrum of mass-selected $\mathrm{Li}_{2} \mathrm{Cu}_{3}(\mathrm{Me})^{n} \mathrm{Bu}_{5}{ }^{-}(m / z=503)$ and its fragment ions produced upon collision-induced dissociation $\left(V_{\mathrm{exc}}=0.21 \mathrm{~V}\right)$.

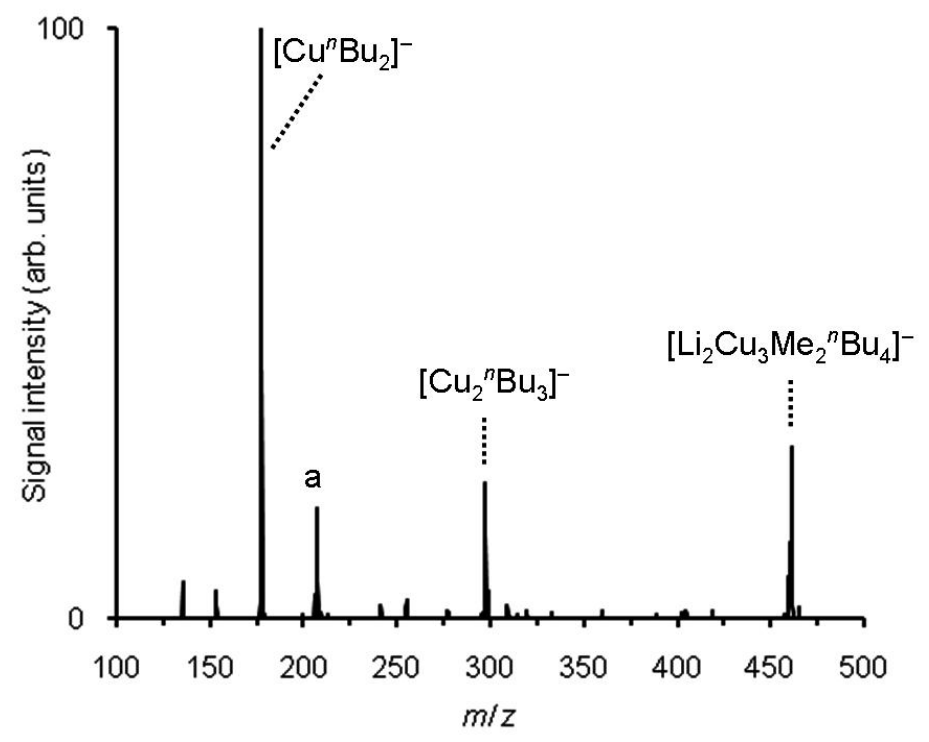

Figure S62. Mass spectrum of mass-selected $\mathrm{Li}_{2} \mathrm{Cu}_{3} \mathrm{Me}_{2}{ }^{n} \mathrm{Bu}_{4}{ }^{-}(m / z=461)$ and its fragment ions produced upon collision-induced dissociation $\left(V_{\mathrm{exc}}=0.19 \mathrm{~V}\right), \mathrm{a}=\mathrm{LiH}_{2} \mathrm{Cu}_{2}(\mathrm{Me})^{n} \mathrm{Bu}^{-}$. 


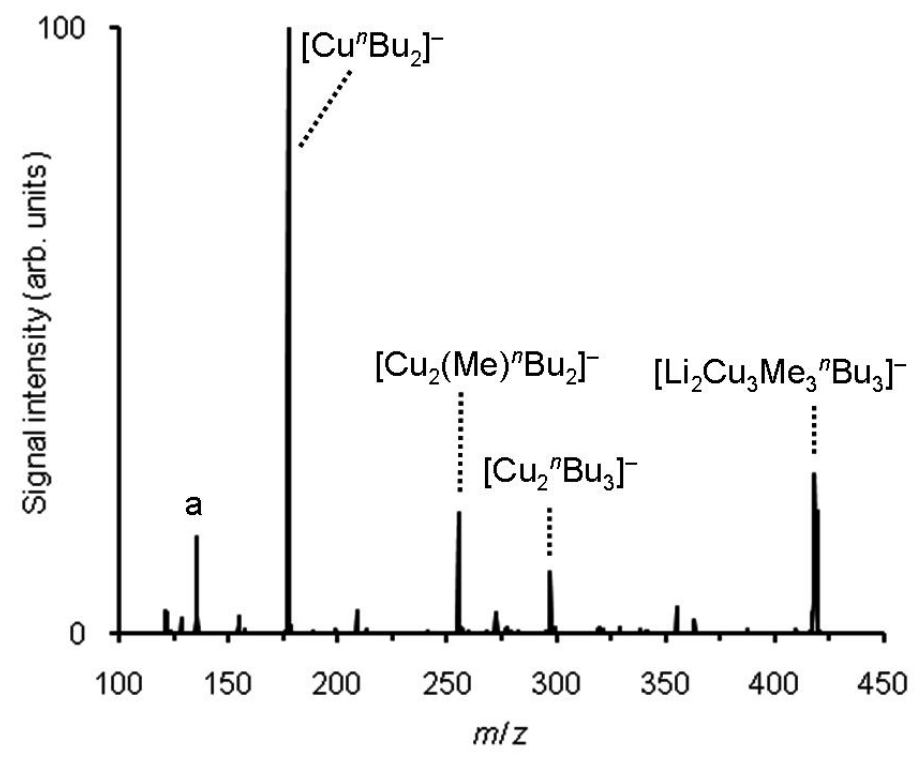

Figure S63. Mass spectrum of mass-selected $\mathrm{Li}_{2} \mathrm{Cu}_{3} \mathrm{Me}_{3}{ }^{n} \mathrm{Bu}_{3}{ }^{-}(m / z=419)$ and its fragment ions produced upon collision-induced dissociation $\left(V_{\mathrm{exc}}=0.16 \mathrm{~V}\right), \mathrm{a}=\mathrm{Cu}(\mathrm{Me})^{n} \mathrm{Bu}^{-}$.

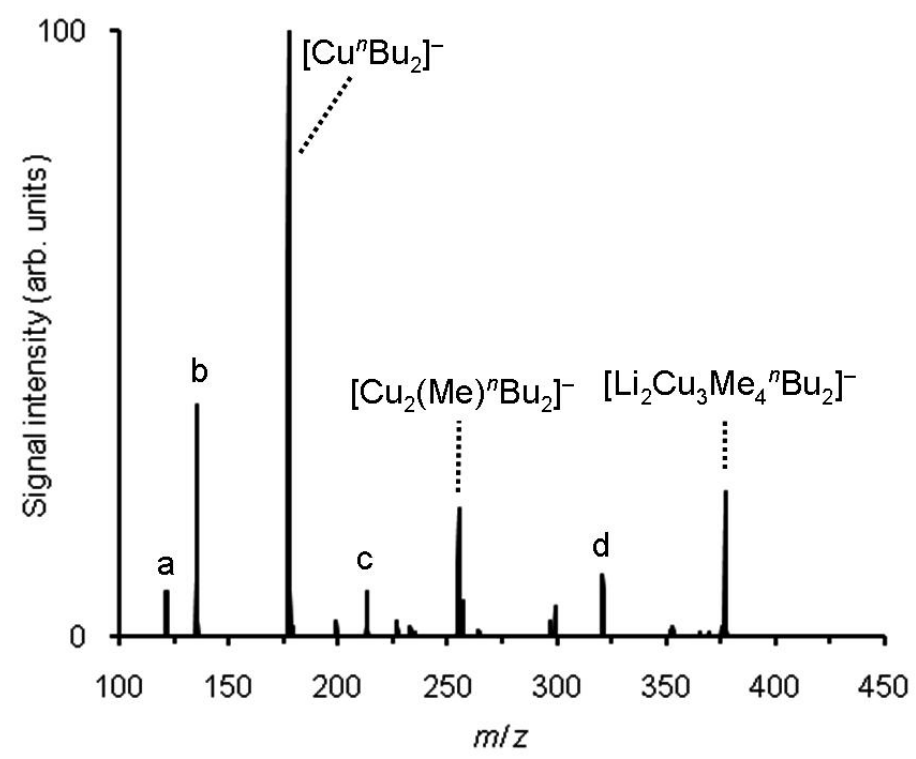

Figure S64. Mass spectrum of mass-selected $\mathrm{Li}_{2} \mathrm{Cu}_{3} \mathrm{Me}_{4}{ }^{n} \mathrm{Bu}_{2}{ }^{-}(m / z=377)$ and its fragment ions produced upon collision-induced dissociation $\left(V_{\mathrm{exc}}=0.34 \mathrm{~V}\right), \mathrm{a}=\mathrm{HCu}^{n} \mathrm{Bu}^{-}, \mathrm{b}=\mathrm{Cu}(\mathrm{Me})^{n} \mathrm{Bu}^{-}, \mathrm{c}=$ $\mathrm{Cu}_{2} \mathrm{Me}_{2}{ }^{n} \mathrm{Bu}^{-}, \mathrm{d}=\mathrm{Li}_{2} \mathrm{HCu}_{3} \mathrm{Me}_{4}{ }^{n} \mathrm{Bu}^{-}$. 


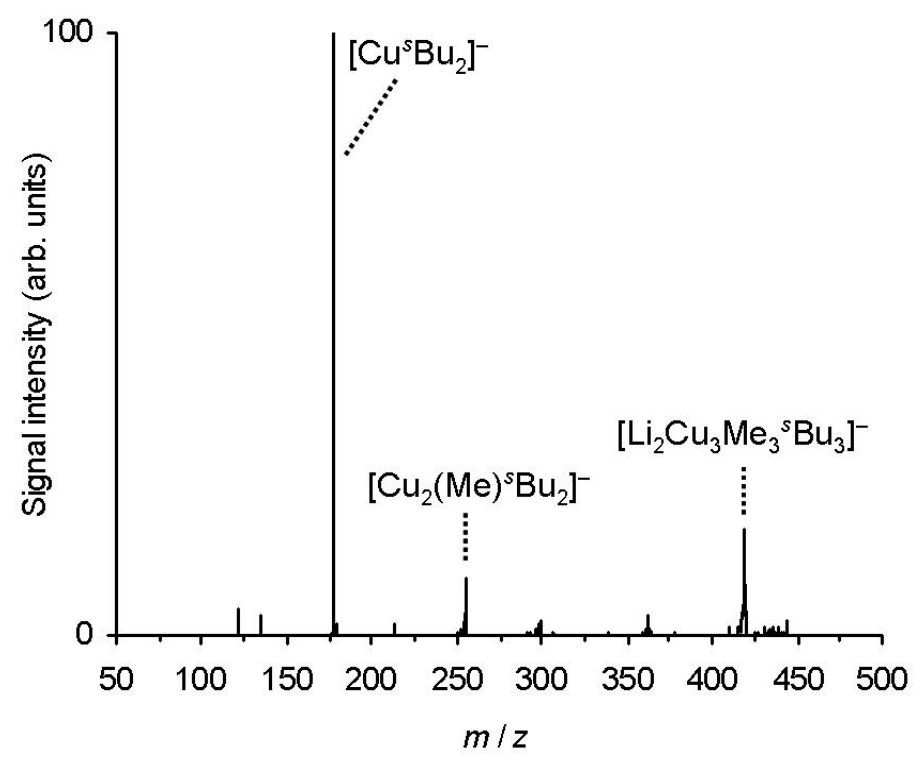

Figure S65. Mass spectrum of mass-selected $\mathrm{Li}_{2} \mathrm{Cu}_{3} \mathrm{Me}_{3}{ }^{s} \mathrm{Bu}_{3}{ }^{-}(m / z=419)$ and its fragment ions produced upon collision-induced dissociation $\left(V_{\mathrm{exc}}=0.17 \mathrm{~V}\right)$.

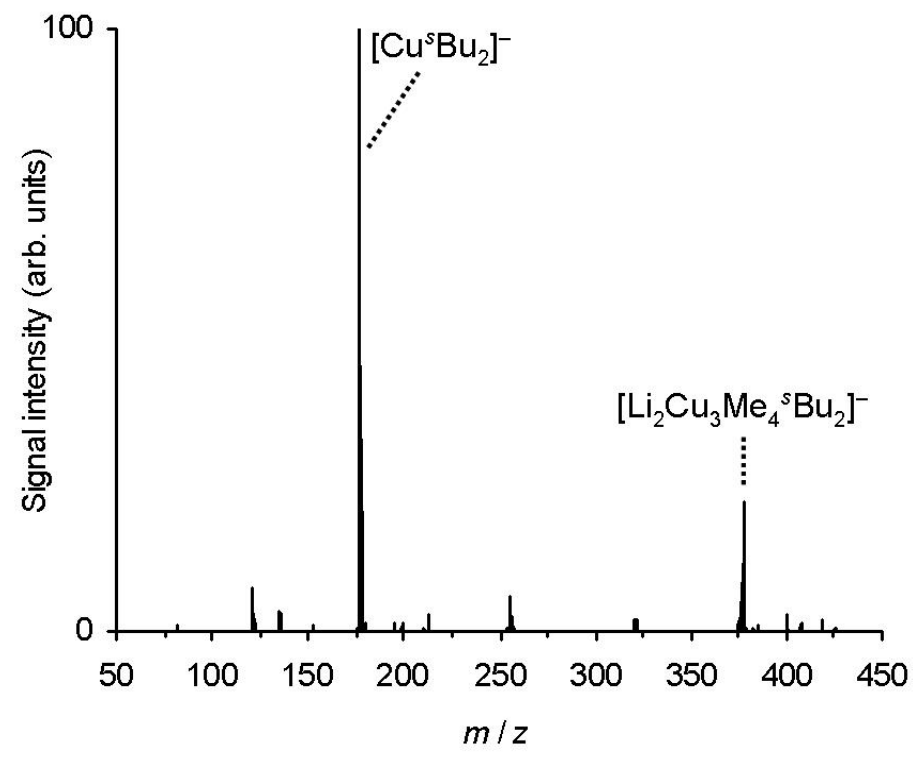

Figure S66. Mass spectrum of mass-selected $\mathrm{Li}_{2} \mathrm{Cu}_{3} \mathrm{Me}_{4}{ }^{s} \mathrm{Bu}_{2}{ }^{-}(m / z=377)$ and its fragment ions produced upon collision-induced dissociation $\left(V_{\mathrm{exc}}=0.30 \mathrm{~V}\right)$. 


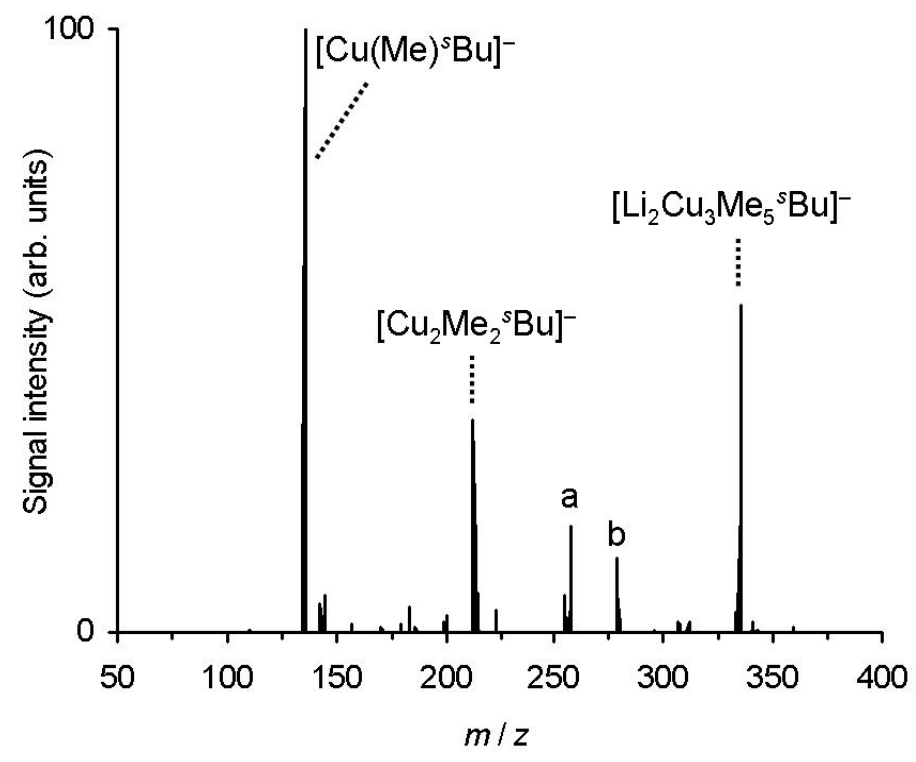

Figure S67. Mass spectrum of mass-selected $\mathrm{Li}_{2} \mathrm{Cu}_{3} \mathrm{Me}_{5}{ }^{s} \mathrm{Bu}^{-}(m / z=335)$ and its fragment ions produced upon collision-induced dissociation $\left(V_{\mathrm{exc}}=0.30 \mathrm{~V}\right)$, a $=\mathrm{Li}_{2} \mathrm{Cu}_{2} \mathrm{Me}_{4}{ }^{s} \mathrm{Bu}^{-}, \mathrm{b}=$ $\mathrm{Li}_{2} \mathrm{HCu}_{3} \mathrm{Me}_{5}^{-}$.

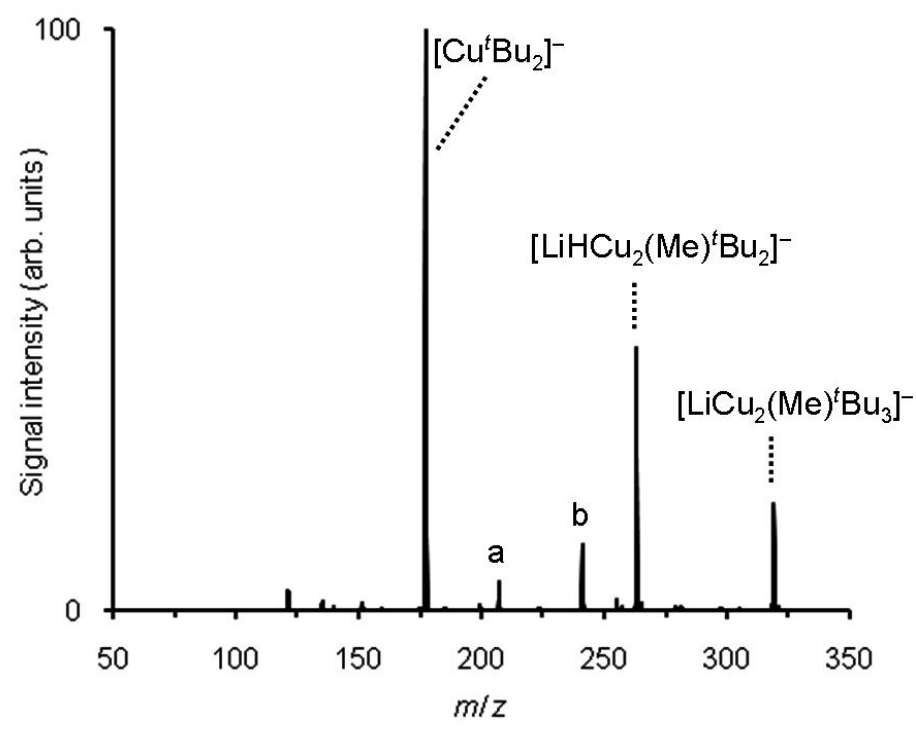

Figure S68. Mass spectrum of mass-selected $\mathrm{LiCu}_{2}(\mathrm{Me})^{t} \mathrm{Bu}_{3}{ }^{-}(m / z=319)$ and its fragment ions produced upon collision-induced dissociation $\left(V_{\mathrm{exc}}=0.35 \mathrm{~V}\right), \mathrm{a}=\mathrm{LiH}_{2} \mathrm{Cu}_{2}(\mathrm{Me})^{t} \mathrm{Bu}^{-}, \mathrm{b}=$ $\mathrm{HCu}_{2}{ }^{t} \mathrm{Bu}_{2}^{-}$. 


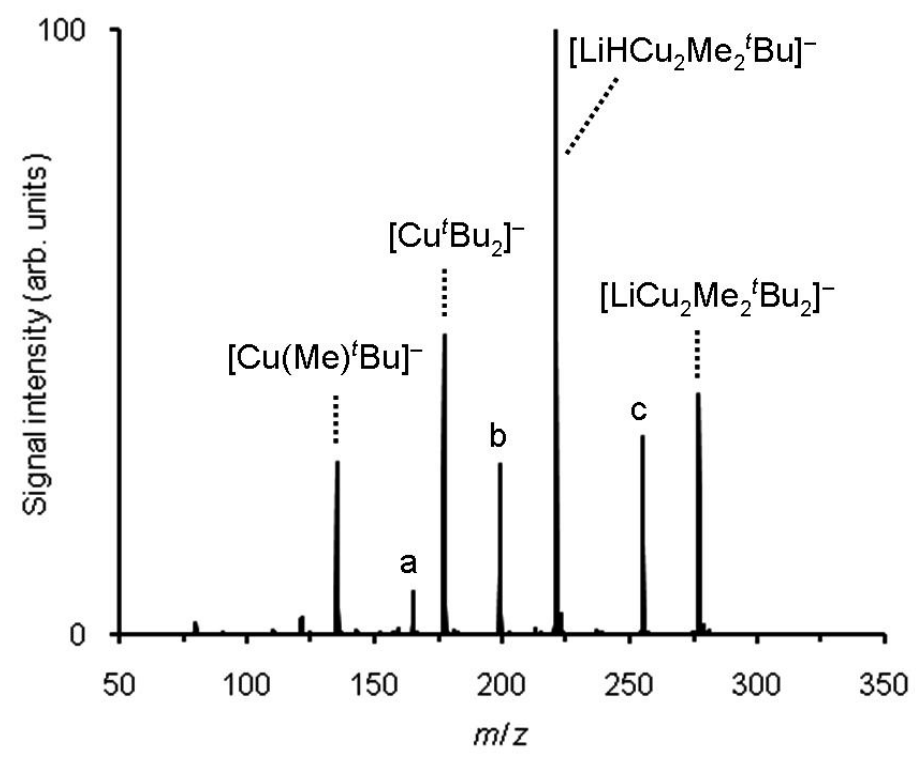

Figure S69. Mass spectrum of mass-selected $\mathrm{LiCu}_{2} \mathrm{Me}_{2}{ }^{t} \mathrm{Bu}_{2}^{-}(m / z=277)$ and its fragment ions produced upon collision-induced dissociation $\left(V_{\mathrm{exc}}=0.35 \mathrm{~V}\right), \mathrm{a}=\mathrm{LiH}_{2} \mathrm{Cu}_{2} \mathrm{Me}_{2}^{-}, \mathrm{b}=$ $\mathrm{HCu}_{2}(\mathrm{Me})^{t} \mathrm{Bu}^{-}, \mathrm{c}=\mathrm{Cu}_{2}(\mathrm{Me})^{t} \mathrm{Bu}_{2}^{-}$.

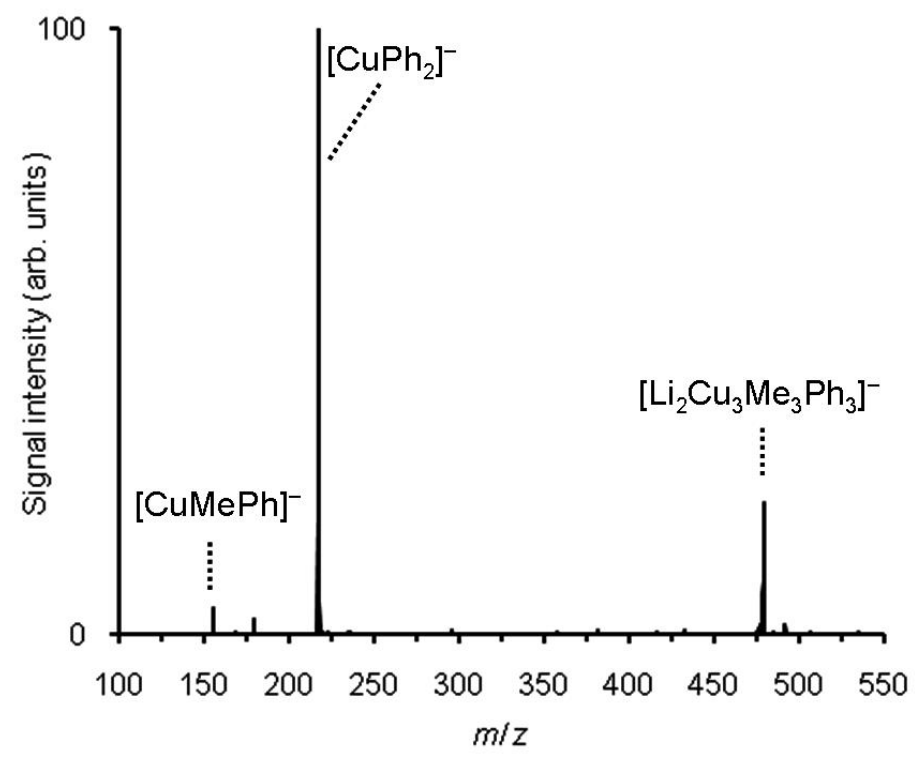

Figure S70. Mass spectrum of mass-selected $\mathrm{Li}_{2} \mathrm{Cu}_{3} \mathrm{Me}_{3} \mathrm{Ph}_{3}{ }^{-}(\mathrm{m} / z=479)$ and its fragment ions produced upon collision-induced dissociation $\left(V_{\mathrm{exc}}=0.17 \mathrm{~V}\right)$. 


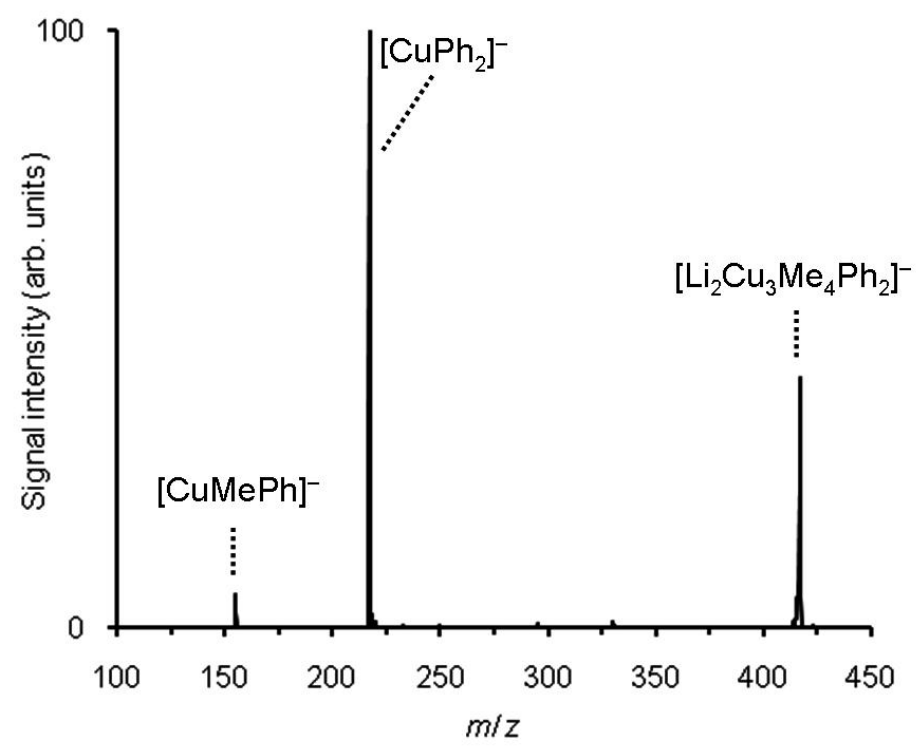

Figure S71. Mass spectrum of mass-selected $\mathrm{Li}_{2} \mathrm{Cu}_{3} \mathrm{Me}_{4} \mathrm{Ph}_{2}{ }^{-}(m / z=417)$ and its fragment ions produced upon collision-induced dissociation $\left(V_{\mathrm{exc}}=0.15 \mathrm{~V}\right)$.

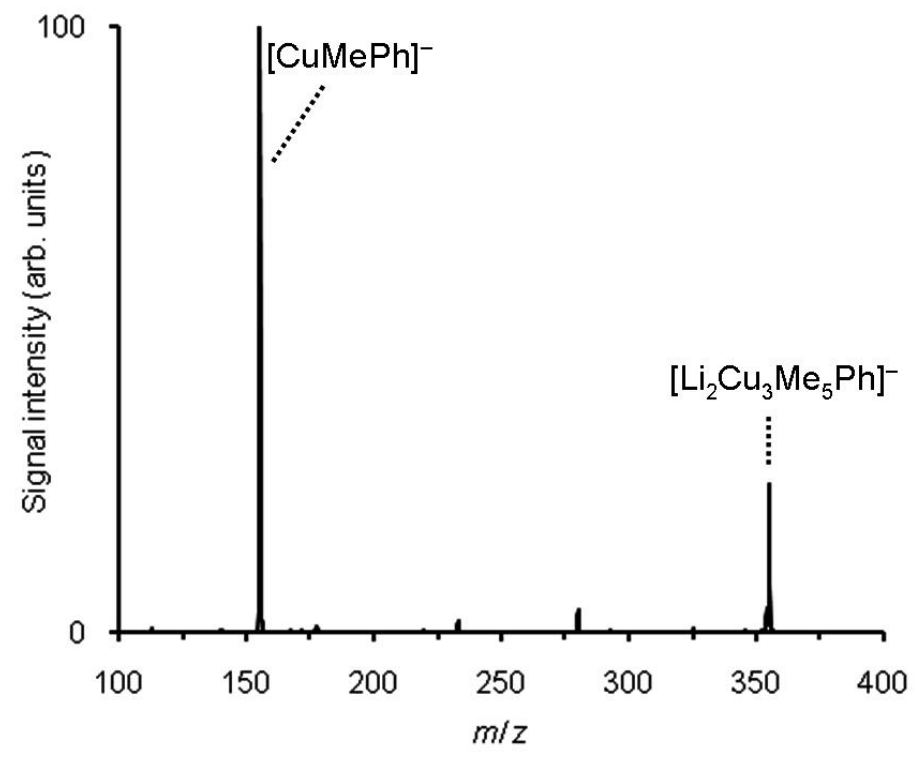

Figure S72. Mass spectrum of mass-selected $\mathrm{Li}_{2} \mathrm{Cu}_{3} \mathrm{Me}_{5} \mathrm{Ph}^{-}(m / z=355)$ and its fragment ions produced upon collision-induced dissociation $\left(V_{\mathrm{exc}}=0.27 \mathrm{~V}\right)$. 
11.) Mass spectra of mass-selected cyanide-containing cuprate anions and their fragment ions

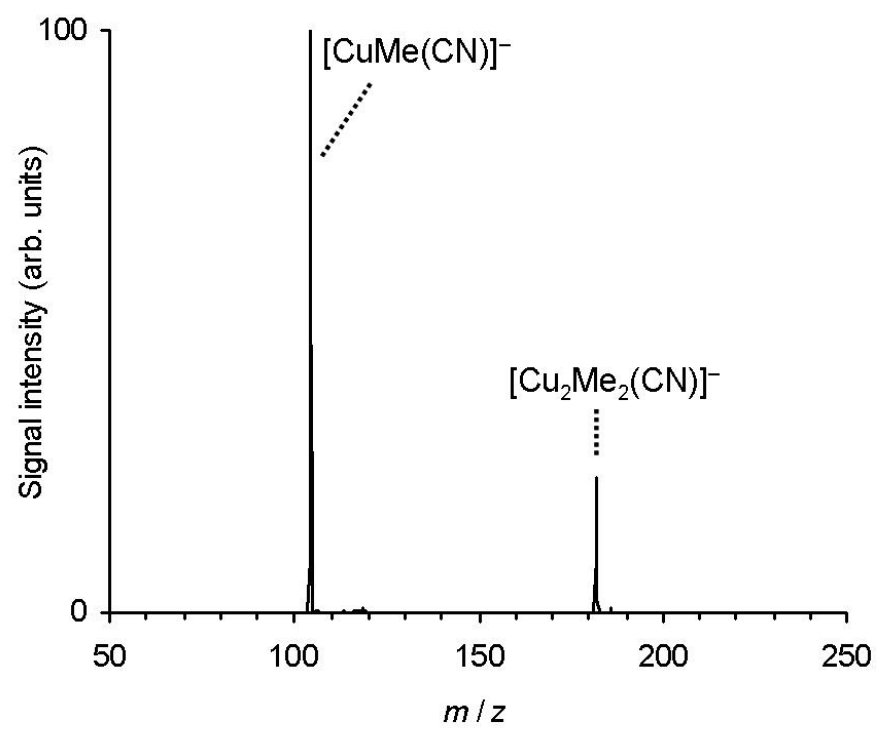

Figure S73. Mass spectrum of mass-selected $\mathrm{Cu}_{2} \mathrm{Me}_{2}(\mathrm{CN})^{-}(m / z=182)$ and its fragment ions produced upon collision-induced dissociation $\left(V_{\mathrm{exc}}=0.30 \mathrm{~V}\right)$.

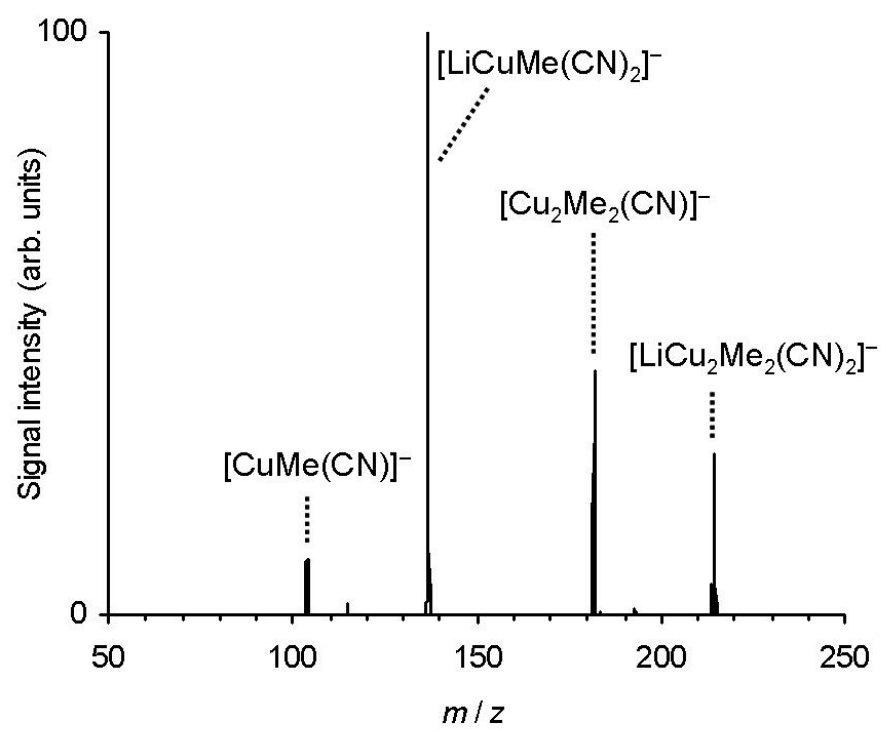

Figure S74. Mass spectrum of mass-selected $\mathrm{LiCu}_{2} \mathrm{Me}_{2}(\mathrm{CN})_{2}{ }^{-}(\mathrm{m} / \mathrm{z}=215)$ and its fragment ions produced upon collision-induced dissociation $\left(V_{\mathrm{exc}}=0.40 \mathrm{~V}\right)$. 


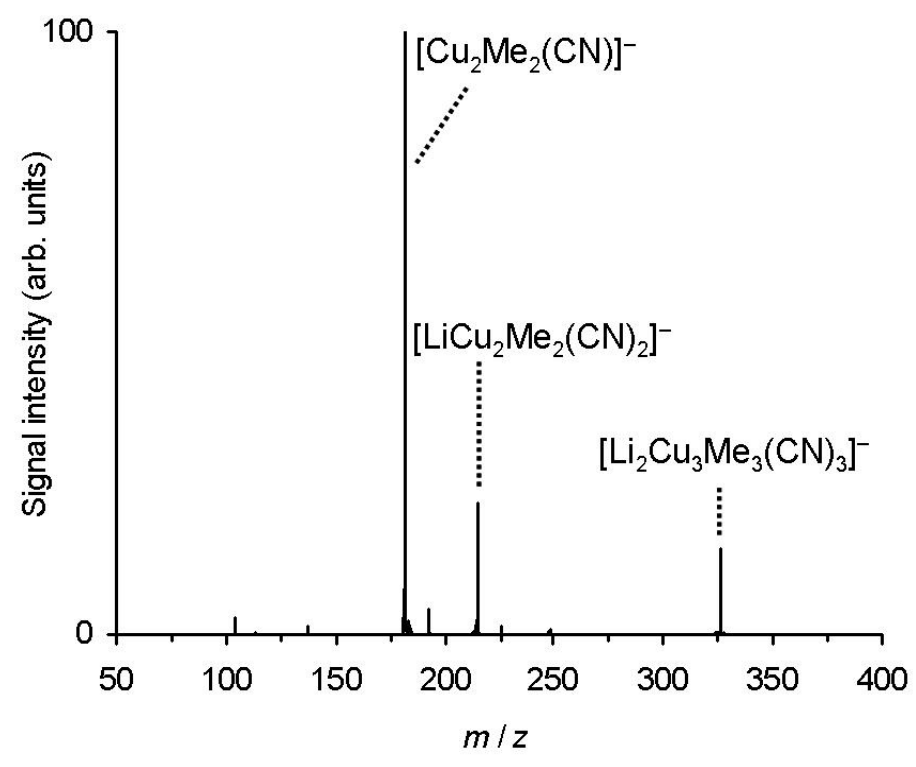

Figure S75. Mass spectrum of mass-selected $\mathrm{Li}_{2} \mathrm{Cu}_{3} \mathrm{Me}_{3}(\mathrm{CN})_{3}{ }^{-}(\mathrm{m} / z=326)$ and its fragment ions produced upon collision-induced dissociation $\left(V_{\mathrm{exc}}=0.30 \mathrm{~V}\right)$.

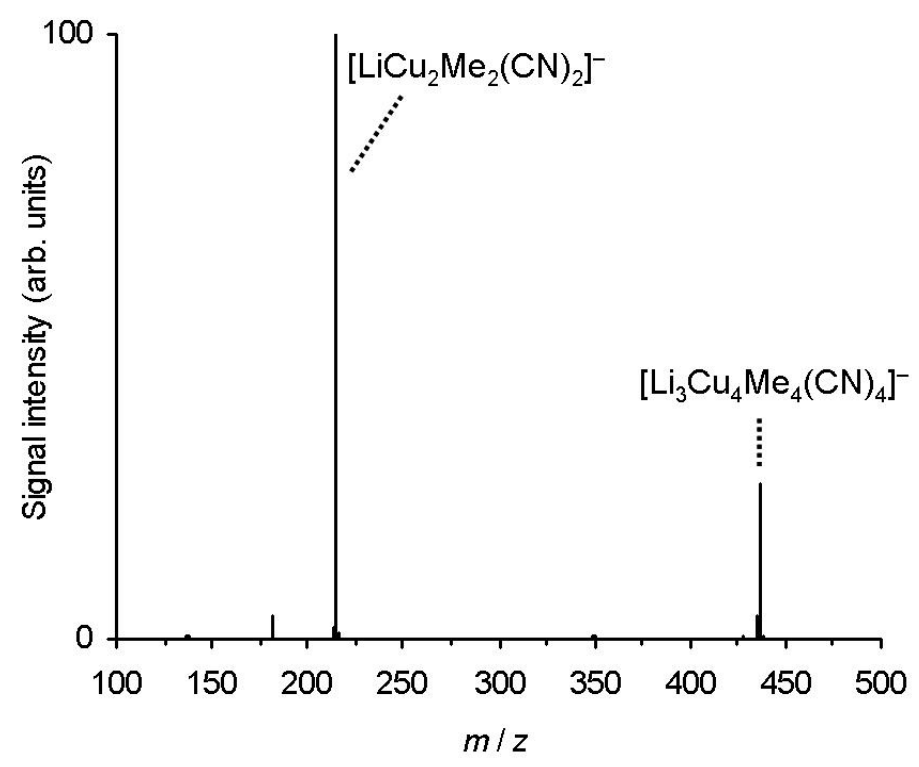

Figure S76. Mass spectrum of mass-selected $\mathrm{Li}_{3} \mathrm{Cu}_{4} \mathrm{Me}_{4}(\mathrm{CN})_{4}{ }^{-}(\mathrm{m} / z=437)$ and its fragment ions produced upon collision-induced dissociation $\left(V_{\mathrm{exc}}=0.13 \mathrm{~V}\right)$. 


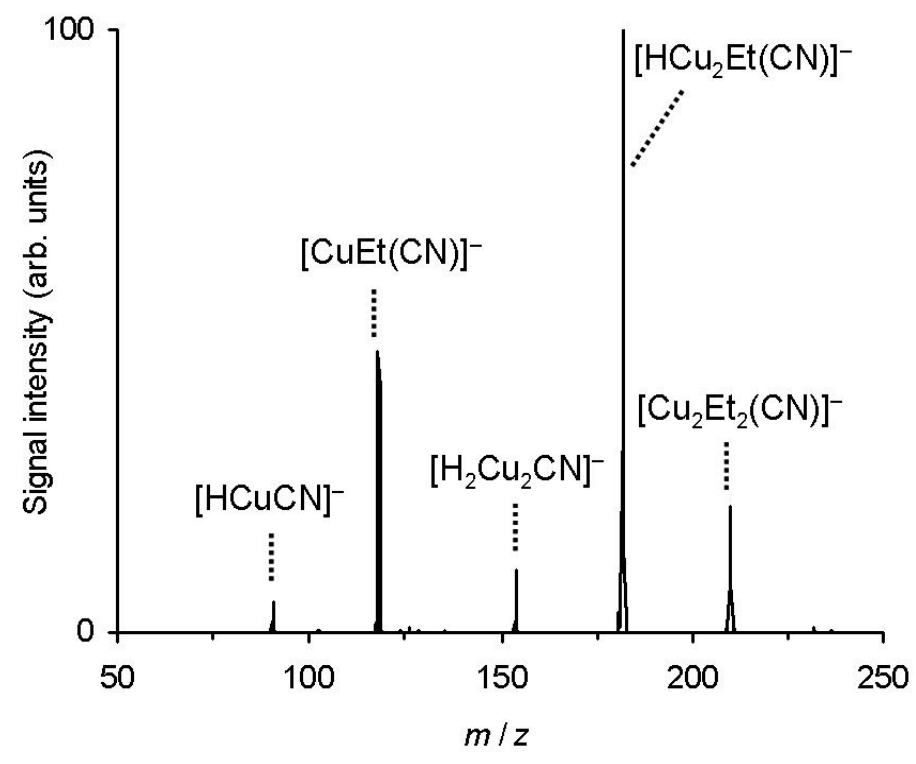

Figure S77. Mass spectrum of mass-selected $\mathrm{Cu}_{2} \mathrm{Et}_{2}(\mathrm{CN})^{-}(\mathrm{m} / z=210)$ and its fragment ions produced upon collision-induced dissociation $\left(V_{\mathrm{exc}}=0.35 \mathrm{~V}\right)$.

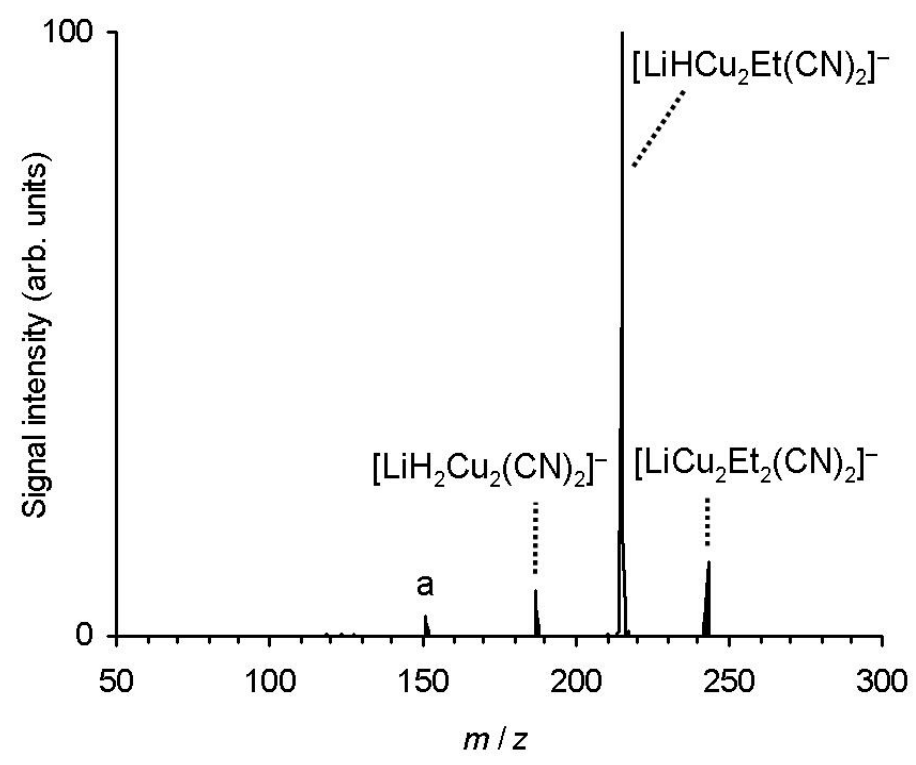

Figure S78. Mass spectrum of mass-selected $\mathrm{LiCu}_{2} \mathrm{Et}_{2}(\mathrm{CN})_{2}{ }^{-}(m / z=243)$ and its fragment ions produced upon collision-induced dissociation $\left(V_{\mathrm{exc}}=0.35 \mathrm{~V}\right), \mathrm{a}=\operatorname{LiCuEt}(\mathrm{CN})_{2}{ }^{-}$. 


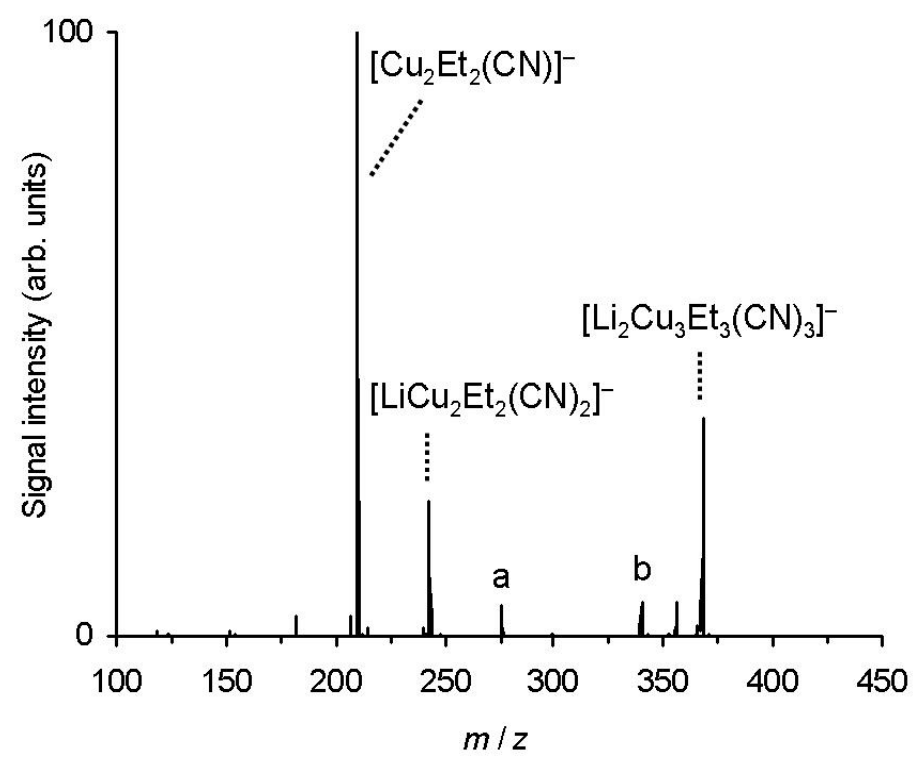

Figure S79. Mass spectrum of mass-selected $\mathrm{Li}_{2} \mathrm{Cu}_{3} \mathrm{Et}_{3}(\mathrm{CN})_{3}{ }^{-}(\mathrm{m} / z=368)$ and its fragment ions produced upon collision-induced dissociation $\left(V_{\mathrm{exc}}=0.30 \mathrm{~V}\right), \mathrm{a}=\mathrm{Li}_{2} \mathrm{Cu}_{2} \mathrm{Et}_{2}(\mathrm{CN})_{3}{ }^{-}, \mathrm{b}=$ $\mathrm{Li}_{2} \mathrm{HCu}_{3} \mathrm{Et}_{2}(\mathrm{CN})_{3}{ }^{-}$.

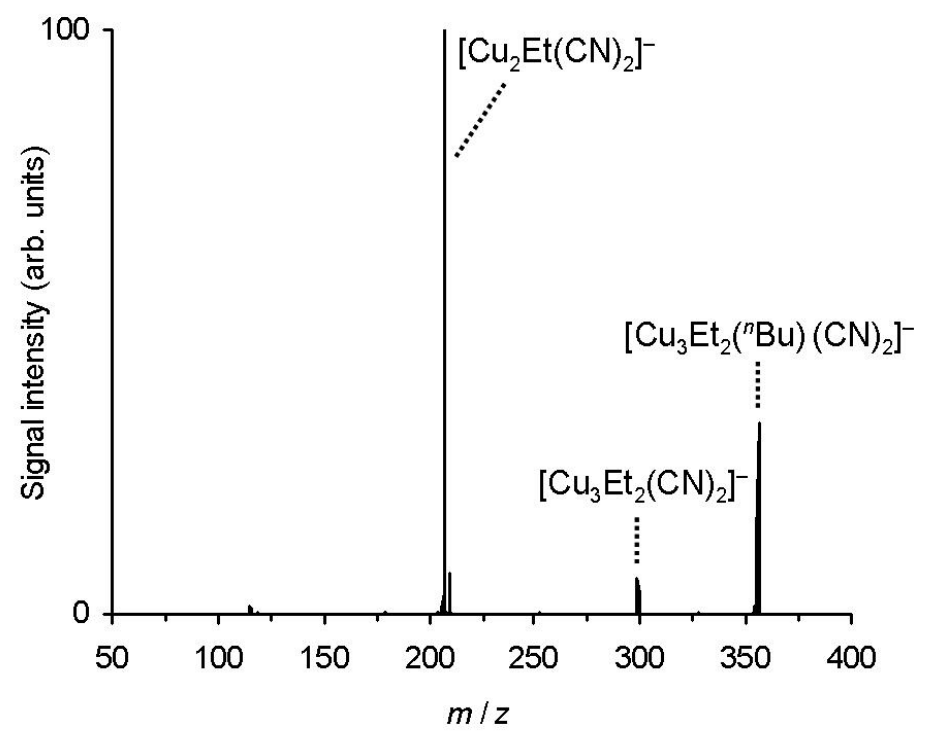

Figure S80. Mass spectrum of mass-selected $\mathrm{Cu}_{3} \mathrm{Et}_{2}\left({ }^{n} \mathrm{Bu}\right)(\mathrm{CN})_{2}{ }^{-}(\mathrm{m} / z=356)$ and its fragment ions produced upon collision-induced dissociation $\left(V_{\mathrm{exc}}=0.25 \mathrm{~V}\right)$. 


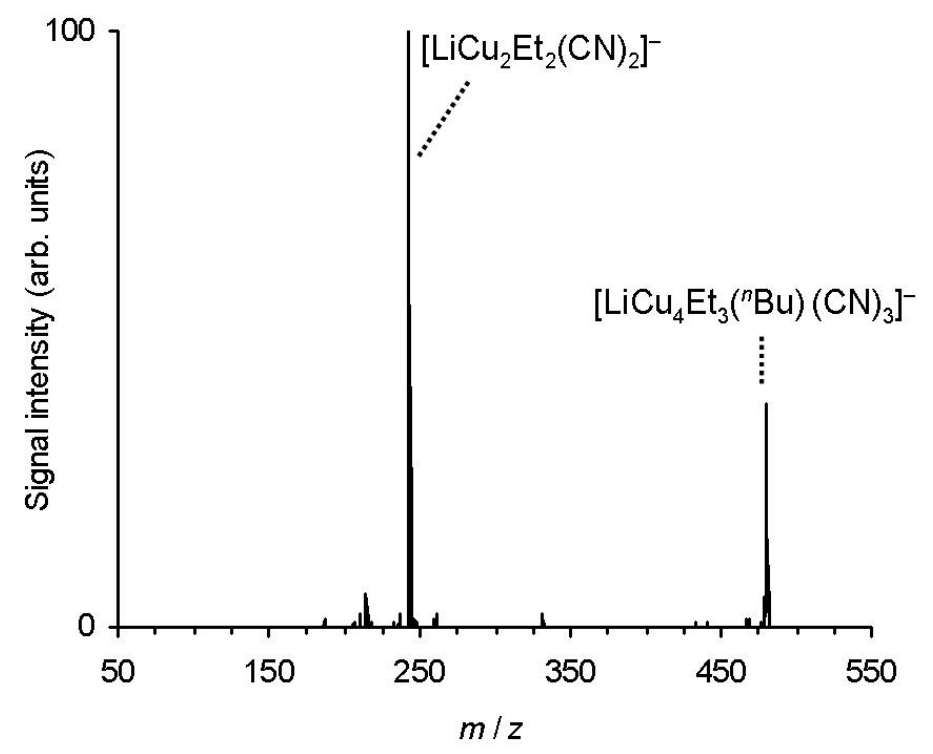

Figure S81. Mass spectrum of mass-selected $\mathrm{LiCu}_{4} \mathrm{Et}_{3}{ }^{n} \mathrm{Bu}(\mathrm{CN})_{3}{ }^{-}(\mathrm{m} / \mathrm{z}=481)$ and its fragment ions produced upon collision-induced dissociation $\left(V_{\mathrm{exc}}=0.16 \mathrm{~V}\right)$.

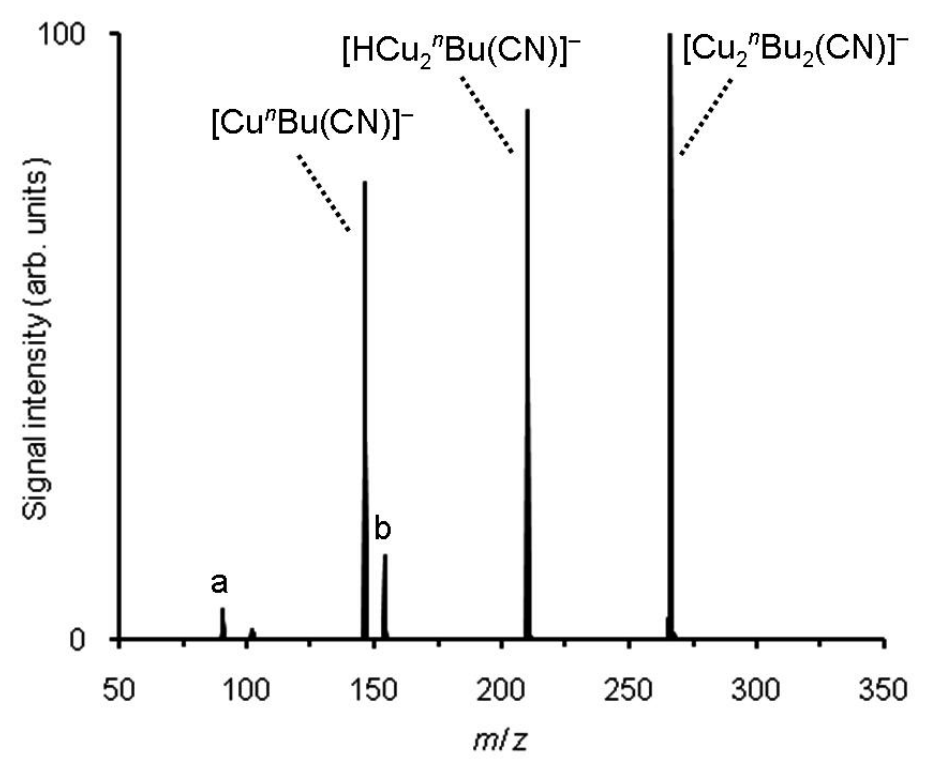

Figure S82. Mass spectrum of mass-selected $\mathrm{Cu}_{2}{ }^{n} \mathrm{Bu}_{2}(\mathrm{CN})^{-}(m / z=266)$ and its fragment ions produced upon collision-induced dissociation $\left(V_{\mathrm{exc}}=0.35 \mathrm{~V}\right), \mathrm{a}=\mathrm{HCu}(\mathrm{CN})^{-}, \mathrm{b}=\mathrm{H}_{2} \mathrm{Cu}_{2}(\mathrm{CN})^{-}$. 


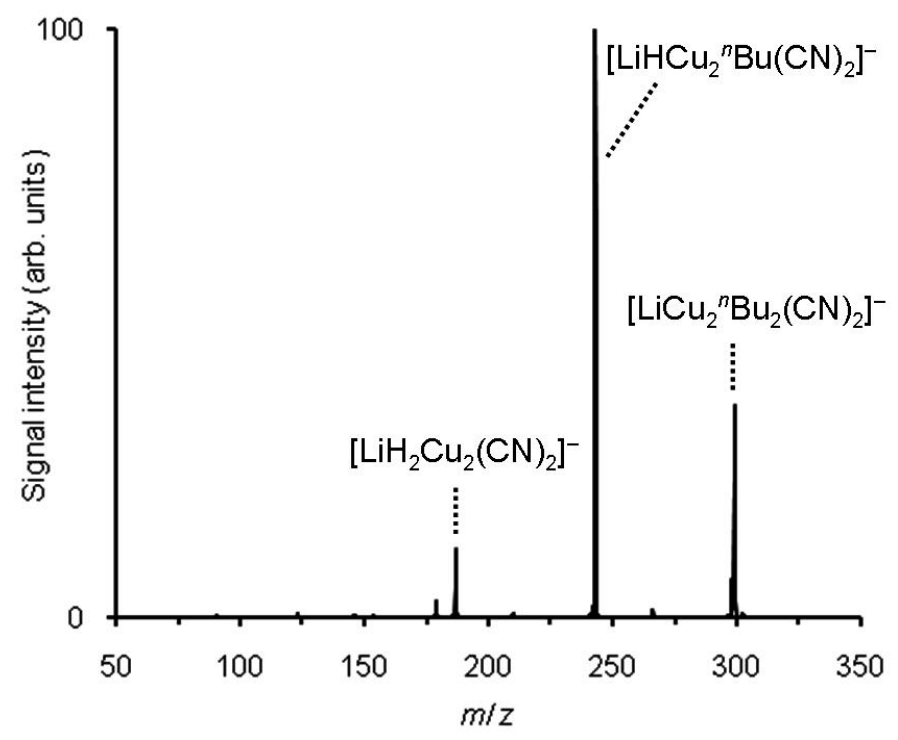

Figure S83. Mass spectrum of mass-selected $\mathrm{LiCu}_{2}{ }^{n} \mathrm{Bu}_{2}(\mathrm{CN})_{2}{ }^{-}(m / z=299)$ and its fragment ions produced upon collision-induced dissociation $\left(V_{\mathrm{exc}}=0.35 \mathrm{~V}\right)$.

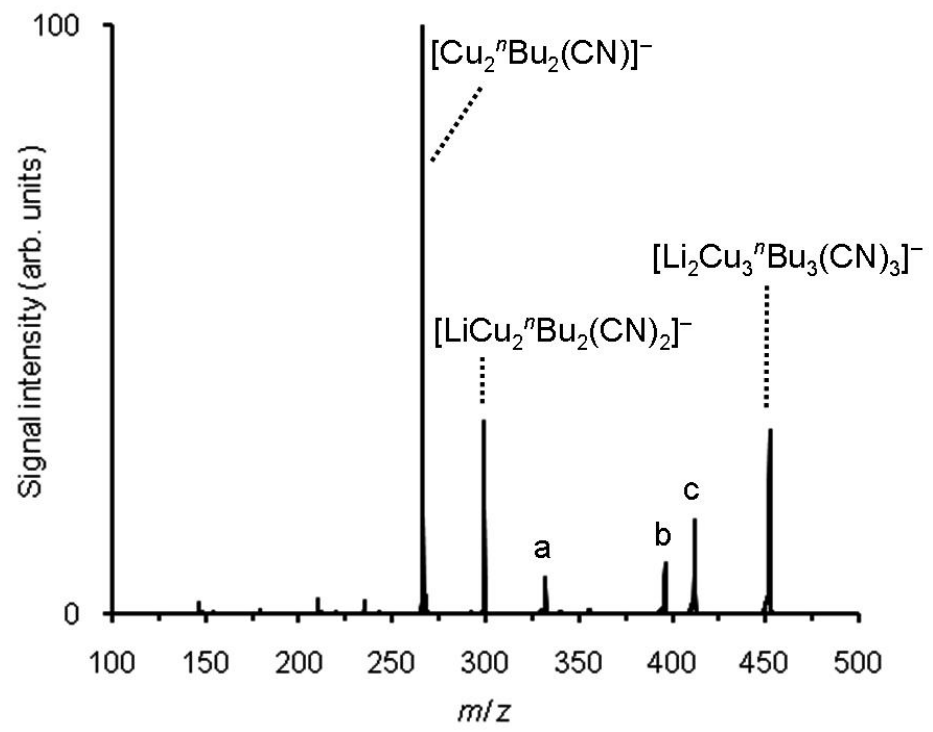

Figure S84. Mass spectrum of mass-selected $\mathrm{Li}_{2} \mathrm{Cu}_{3}{ }^{n} \mathrm{Bu}_{3}(\mathrm{CN})_{3}{ }^{-}(m / z=452)$ and its fragment ions produced upon collision-induced dissociation $\left(V_{\mathrm{exc}}=0.20 \mathrm{~V}\right), \mathrm{a}=\mathrm{Li}_{2} \mathrm{Cu}_{2}{ }^{n} \mathrm{Bu}_{2}(\mathrm{CN})_{3}{ }^{-}, \mathrm{b}=$ $\mathrm{Li}_{2} \mathrm{HCu}_{3}{ }^{n} \mathrm{Bu}_{2}(\mathrm{CN})_{3}{ }^{-}, \mathrm{c}=\mathrm{Cu}_{3}{ }^{n} \mathrm{Bu}_{3}(\mathrm{CN})_{2}{ }^{-}$. The latter does not correspond to a fragment ion but instead results from imperfect mass selection. 


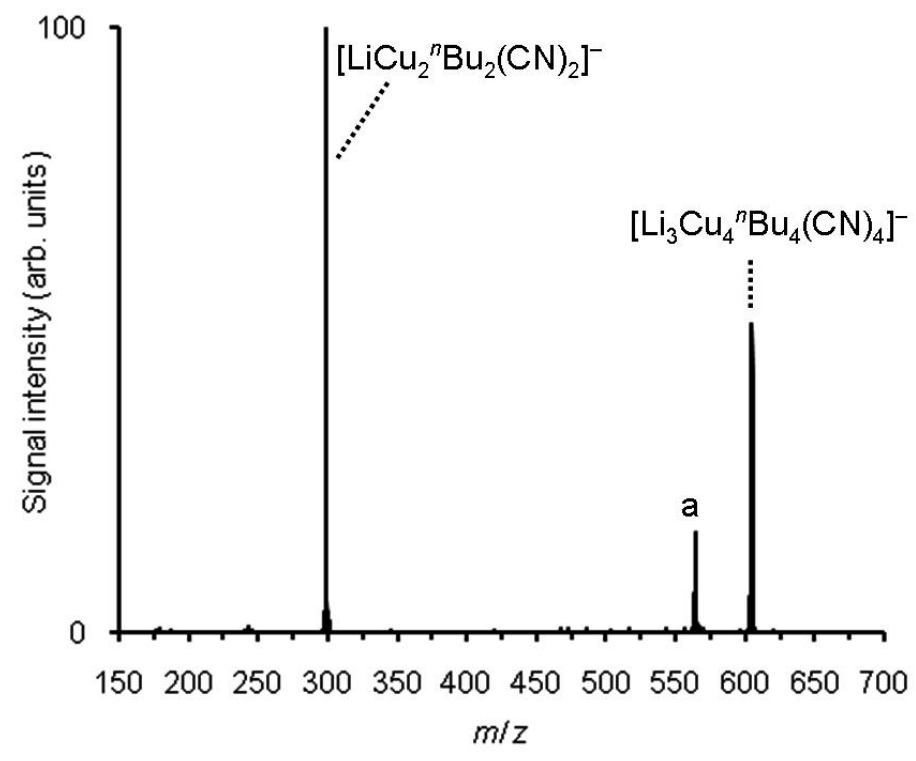

Figure S85. Mass spectrum of mass-selected $\mathrm{Li}_{3} \mathrm{Cu}_{4}{ }^{n} \mathrm{Bu}_{4}(\mathrm{CN})_{4}{ }^{-}(\mathrm{m} / z=605)$ and its fragment ions produced upon collision-induced dissociation $\left(V_{\mathrm{exc}}=0.15 \mathrm{~V}\right), \mathrm{a}=\mathrm{LiCu}_{4}{ }^{n} \mathrm{Bu}_{4}(\mathrm{CN})_{3}{ }^{-}$. The latter does not correspond to a fragment ion but instead results from imperfect mass selection.

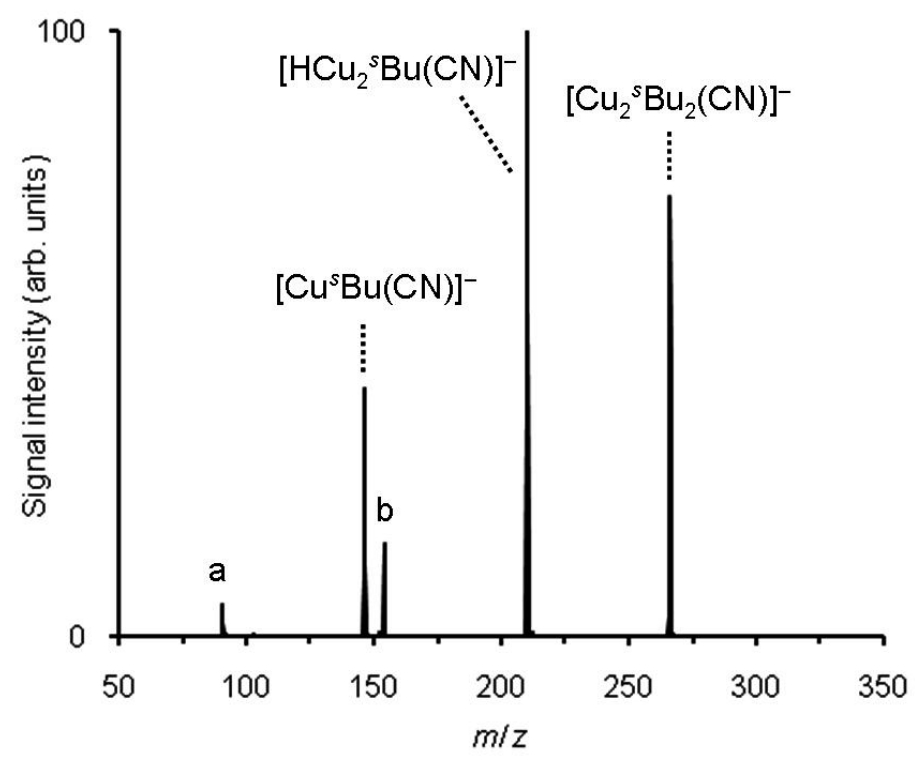

Figure S86. Mass spectrum of mass-selected $\mathrm{Cu}_{2}{ }^{5} \mathrm{Bu}_{2} \mathrm{CN}^{-}(m / z=266)$ and its fragment ions produced upon collision-induced dissociation $\left(V_{\mathrm{exc}}=0.35 \mathrm{~V}\right), \mathrm{a}=\mathrm{HCu}(\mathrm{CN})^{-}, \mathrm{b}=\mathrm{H}_{2} \mathrm{Cu}_{2}(\mathrm{CN})^{-}$. 


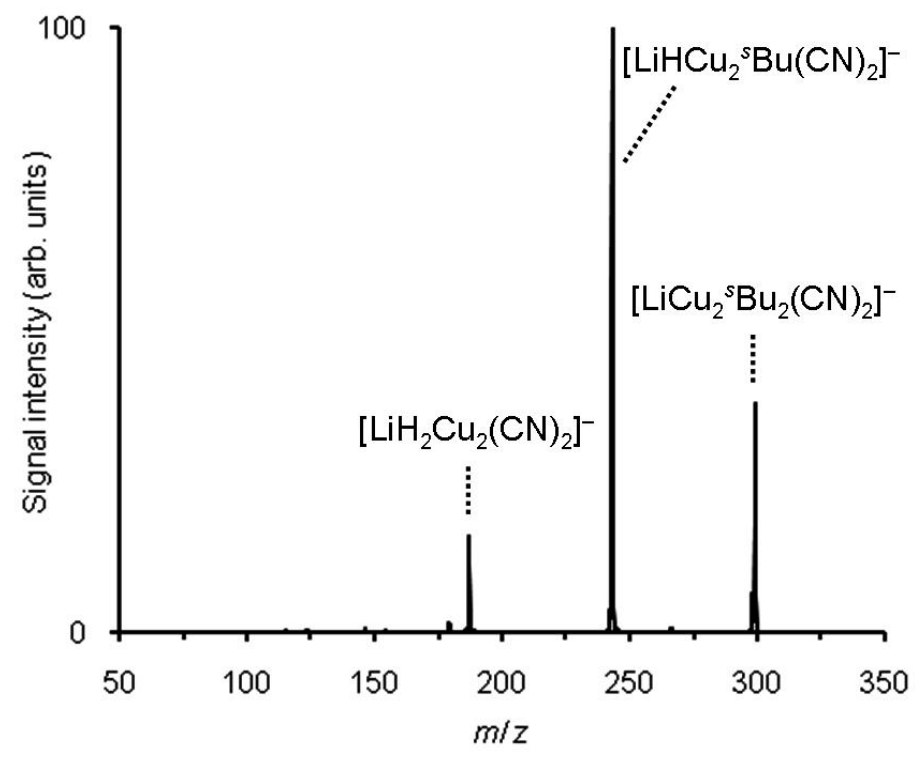

Figure S87. Mass spectrum of mass-selected $\mathrm{LiCu}_{2}{ }^{s} \mathrm{Bu}_{2}(\mathrm{CN})_{2}{ }^{-}(m / z=299)$ and its fragment ions produced upon collision-induced dissociation $\left(V_{\mathrm{exc}}=0.35 \mathrm{~V}\right)$.

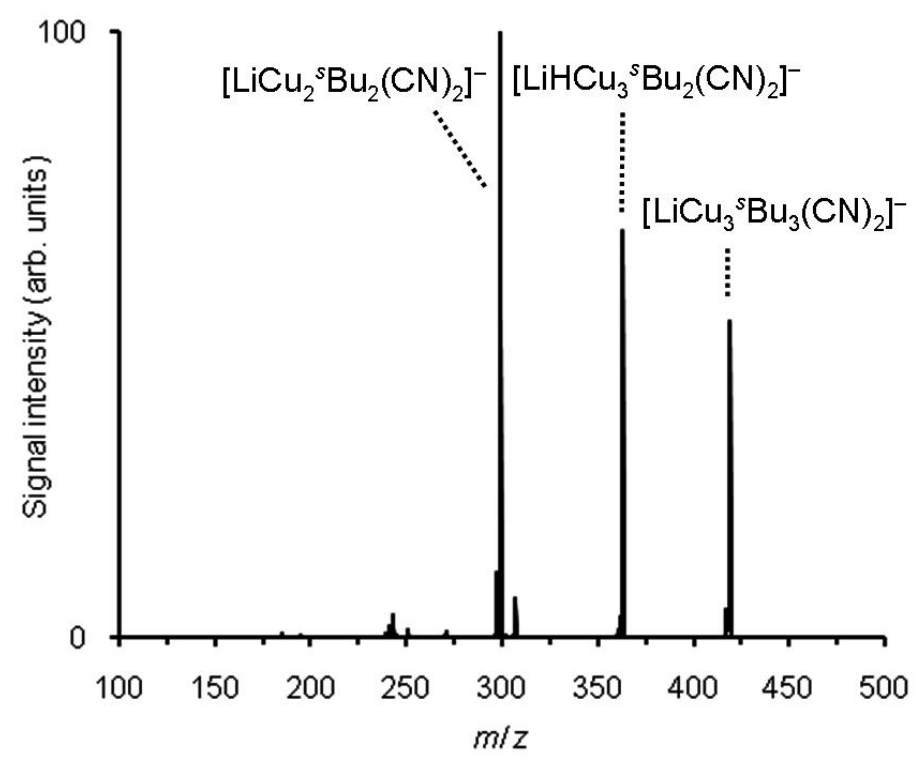

Figure S88. Mass spectrum of mass-selected $\mathrm{LiCu}_{3}{ }^{s} \mathrm{Bu}_{3}(\mathrm{CN})_{2}{ }^{-}(m / z=419)$ and its fragment ions produced upon collision-induced dissociation $\left(V_{\mathrm{exc}}=0.17 \mathrm{~V}\right)$. 


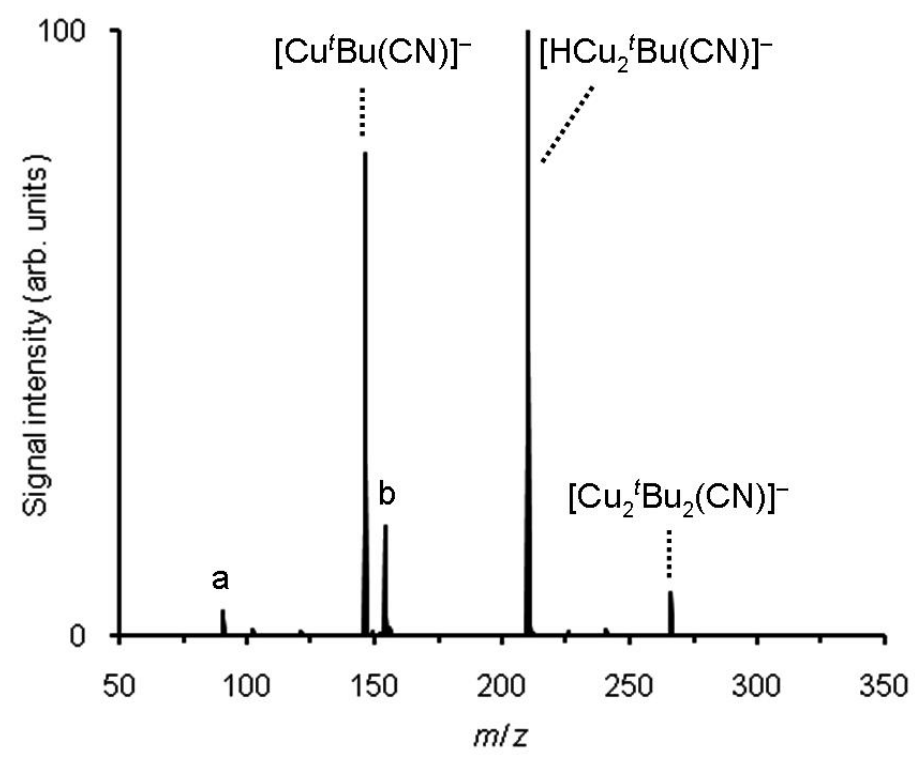

Figure S89. Mass spectrum of mass-selected $\mathrm{Cu}_{2}{ }^{t} \mathrm{Bu}_{2}(\mathrm{CN})^{-}(m / z=266)$ and its fragment ions produced upon collision-induced dissociation $\left(V_{\text {exc }}=0.40 \mathrm{~V}\right), \mathrm{a}=\mathrm{HCu}(\mathrm{CN})^{-}, \mathrm{b}=\mathrm{H}_{2} \mathrm{Cu}_{2}(\mathrm{CN})^{-}$.

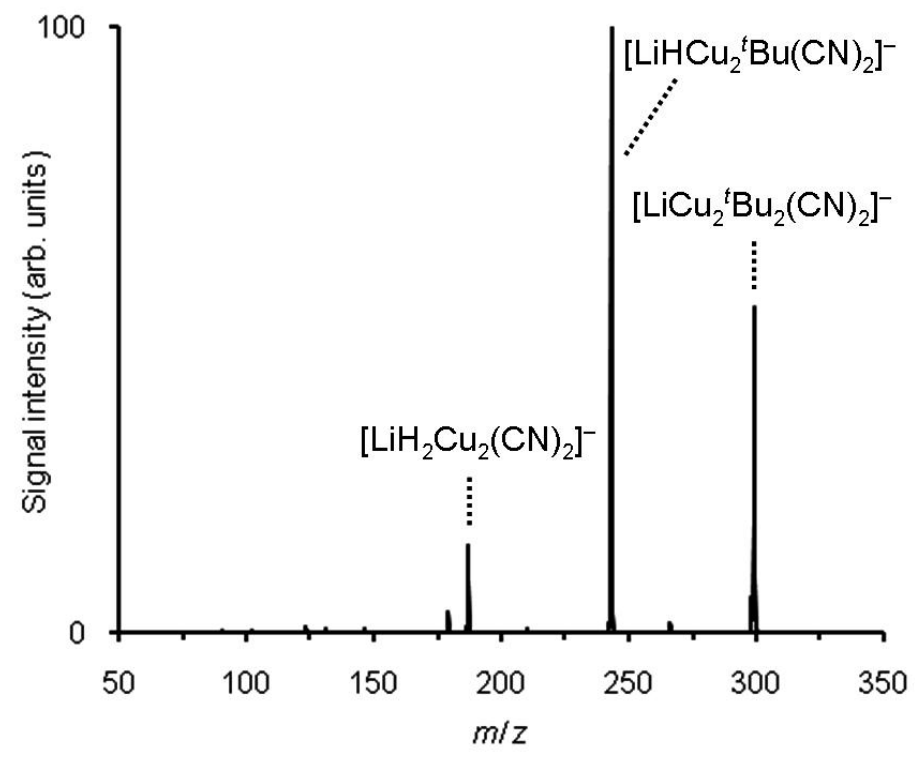

Figure S90. Mass spectrum of mass-selected $\mathrm{LiCu}_{2}{ }^{t} \mathrm{Bu}_{2}(\mathrm{CN})_{2}{ }^{-}(m / z=299)$ and its fragment ions produced upon collision-induced dissociation $\left(V_{\mathrm{exc}}=0.35 \mathrm{~V}\right)$. 


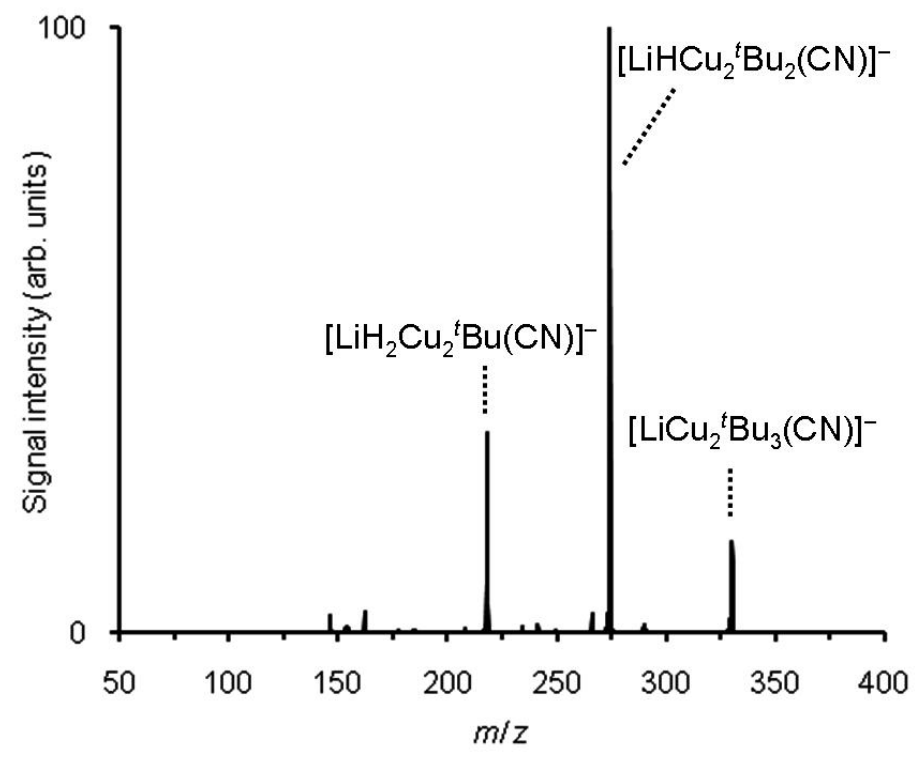

Figure S91. Mass spectrum of mass-selected $\mathrm{LiCu}_{2}{ }^{t} \mathrm{Bu}_{3}(\mathrm{CN})^{-}(m / z=330)$ and its fragment ions produced upon collision-induced dissociation $\left(V_{\mathrm{exc}}=0.35 \mathrm{~V}\right)$.

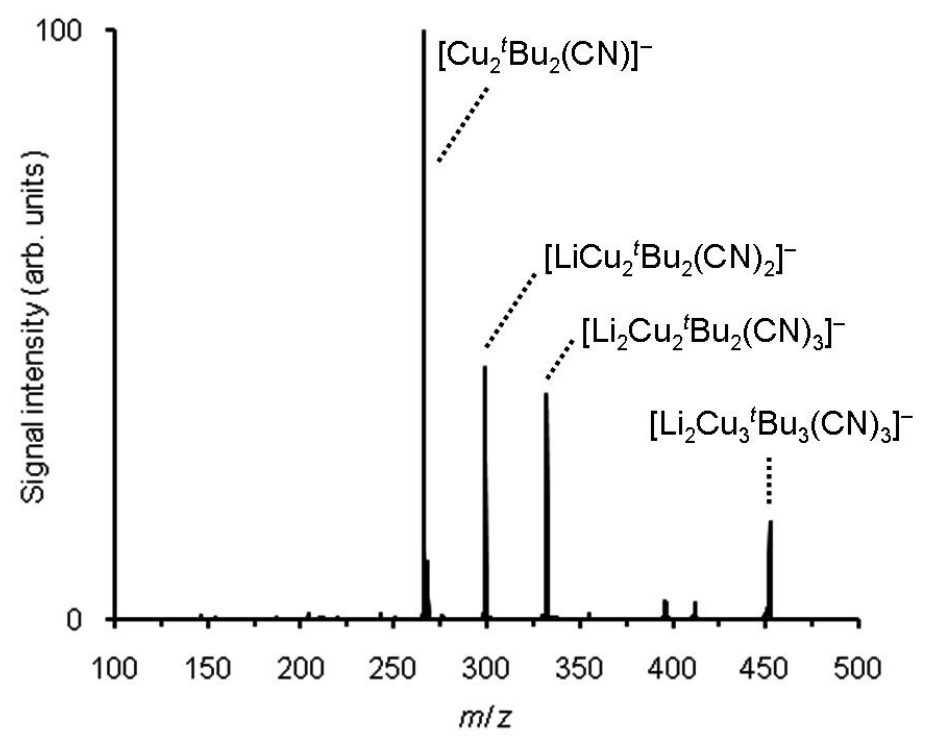

Figure S92. Mass spectrum of mass-selected $\mathrm{Li}_{2} \mathrm{Cu}_{3}{ }^{t} \mathrm{Bu}_{3}(\mathrm{CN})_{3}{ }^{-}(m / z=452)$ and its fragment ions produced upon collision-induced dissociation $\left(V_{\mathrm{exc}}=0.20 \mathrm{~V}\right)$. 


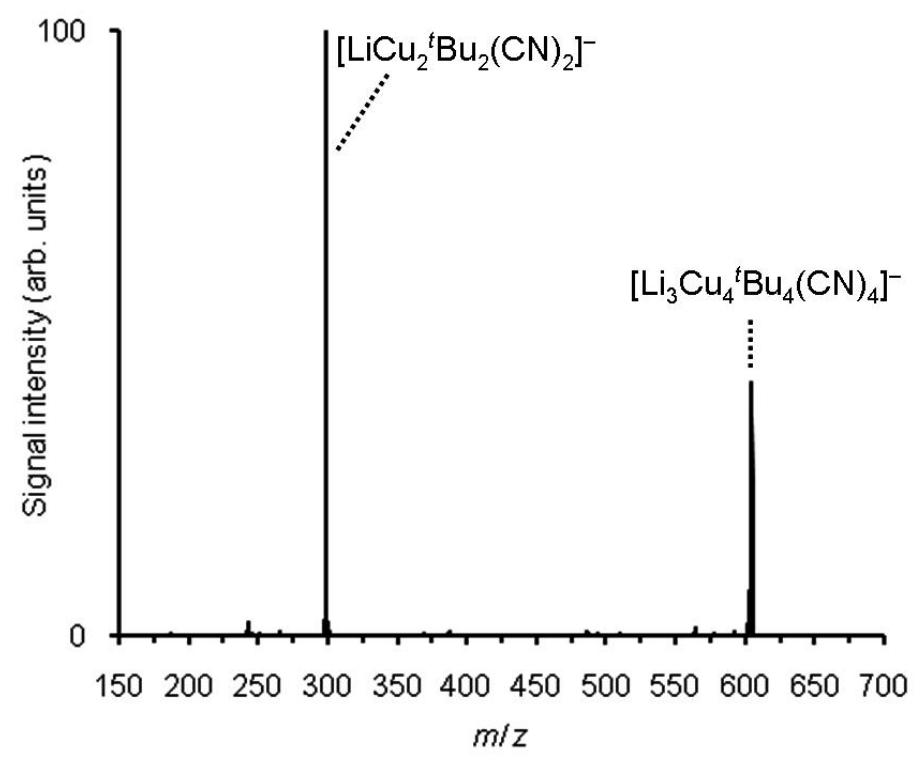

Figure S93. Mass spectrum of mass-selected $\mathrm{Li}_{3} \mathrm{Cu}_{4}{ }^{t} \mathrm{Bu}_{4}(\mathrm{CN})_{4}{ }^{-}(m / z=605)$ and its fragment ions produced upon collision-induced dissociation $\left(V_{\mathrm{exc}}=0.15 \mathrm{~V}\right)$.

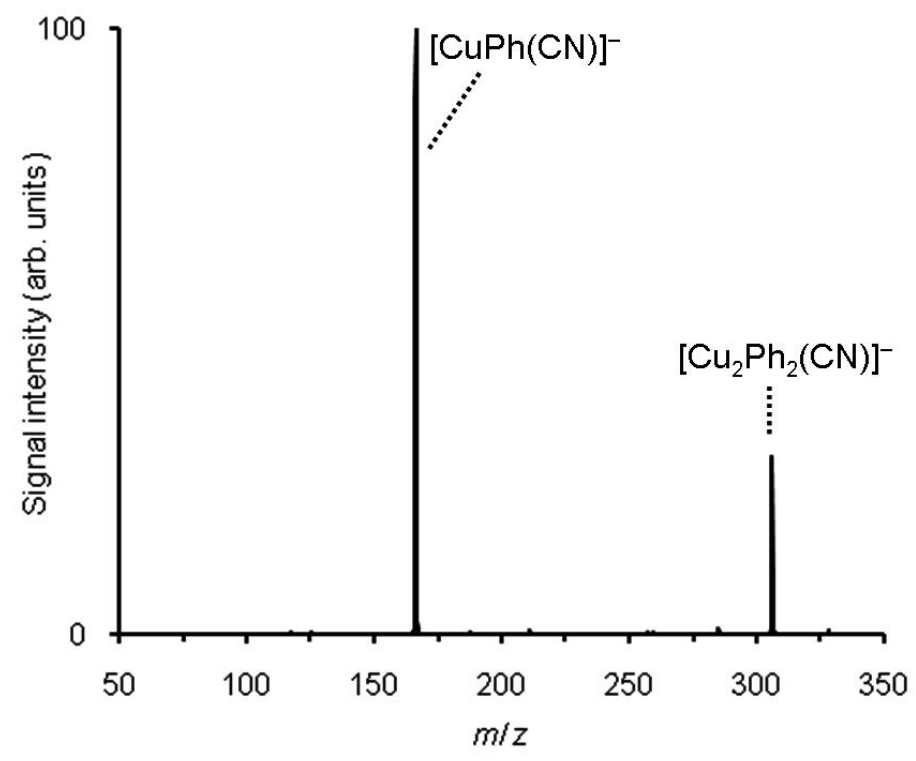

Figure S94. Mass spectrum of mass-selected $\mathrm{Cu}_{2} \mathrm{Ph}_{2}(\mathrm{CN})^{-}(\mathrm{m} / z=306)$ and its fragment ions produced upon collision-induced dissociation $\left(V_{\mathrm{exc}}=0.45 \mathrm{~V}\right)$. 


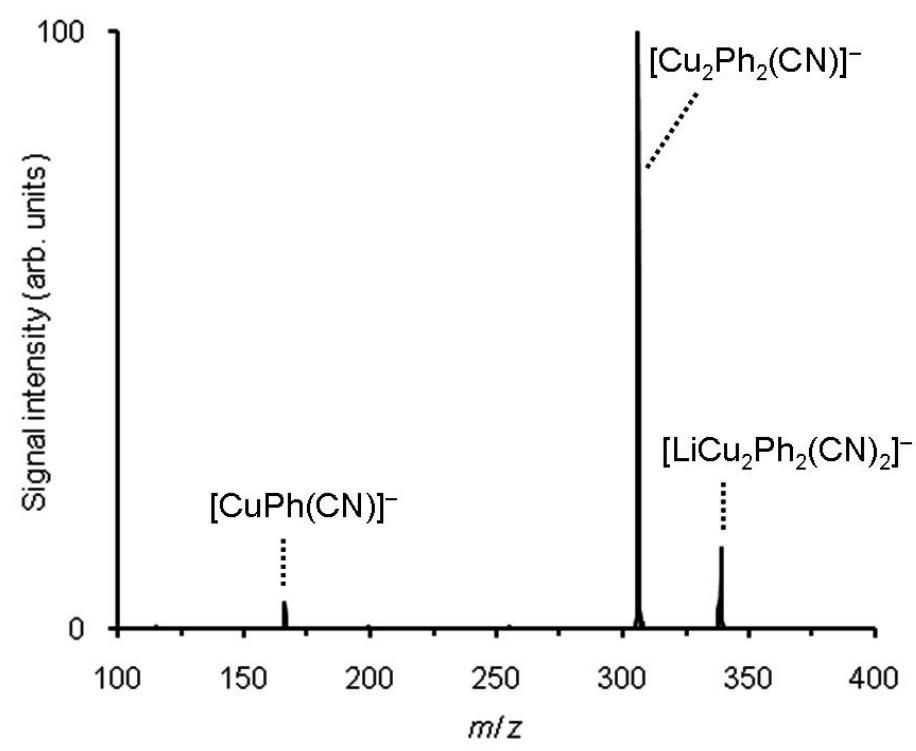

Figure S95. Mass spectrum of mass-selected $\mathrm{LiCu}_{2} \mathrm{Ph}_{2}(\mathrm{CN})_{2}{ }^{-}(m / z=339)$ and its fragment ions produced upon collision-induced dissociation $\left(V_{\mathrm{exc}}=0.45 \mathrm{~V}\right)$.

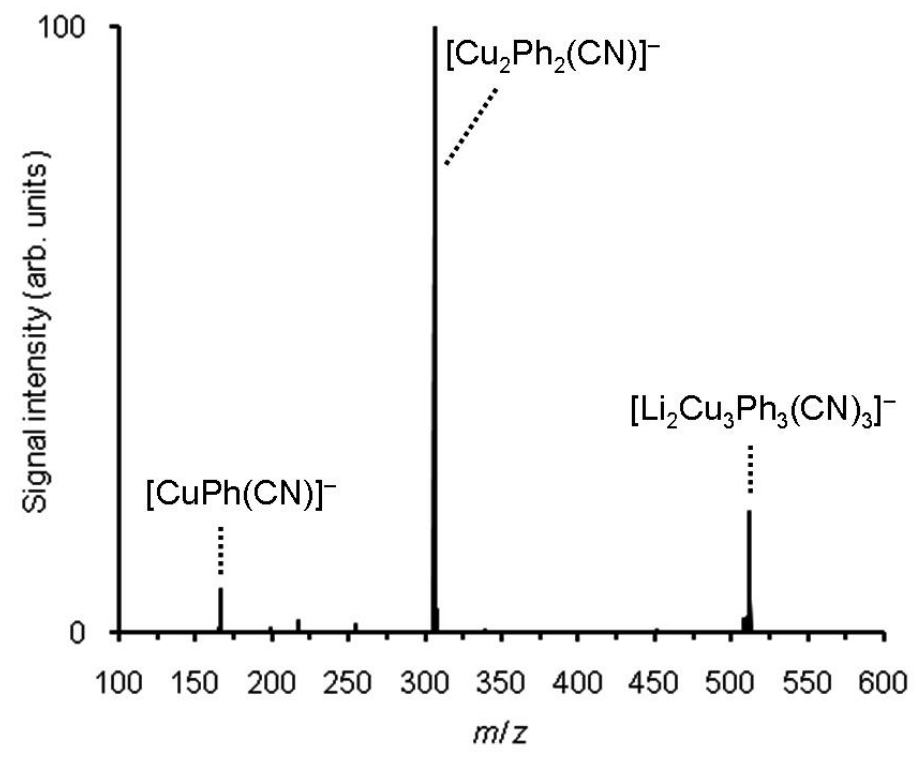

Figure S96. Mass spectrum of mass-selected $\mathrm{Li}_{2} \mathrm{Cu}_{3} \mathrm{Ph}_{3}(\mathrm{CN})_{3}{ }^{-}(\mathrm{m} / z=512)$ and its fragment ions produced upon collision-induced dissociation $\left(V_{\mathrm{exc}}=0.20 \mathrm{~V}\right)$. 


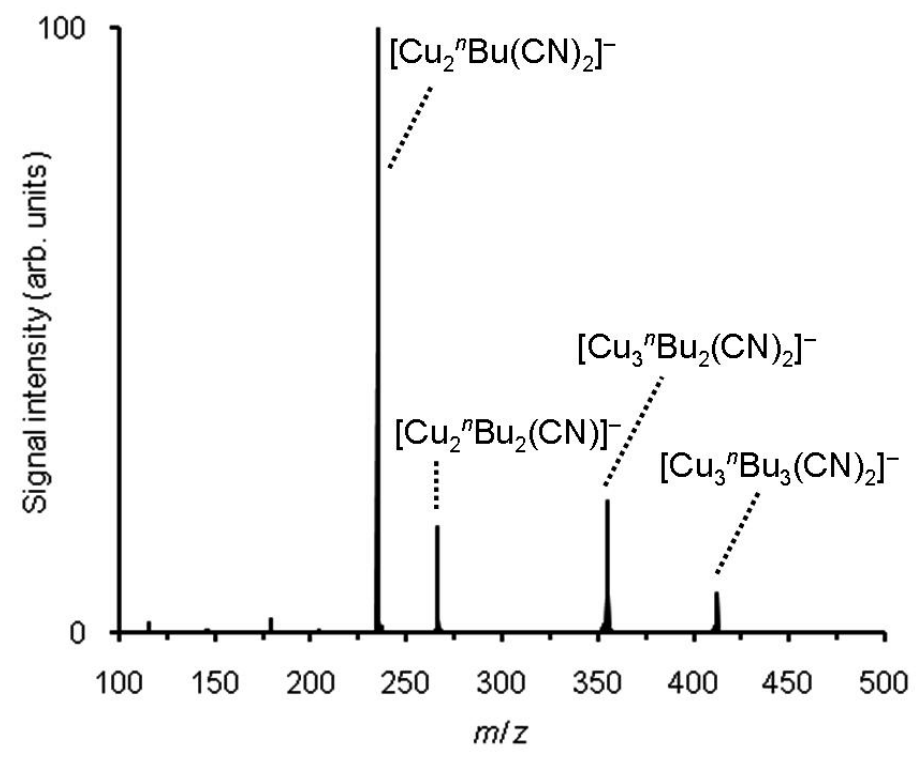

Figure S97. Mass spectrum of mass-selected $\mathrm{Cu}_{3}{ }^{n} \mathrm{Bu}_{3}(\mathrm{CN})_{2}{ }^{-}(m / z=412)$ and its fragment ions produced upon collision-induced dissociation $\left(V_{\mathrm{exc}}=0.20 \mathrm{~V}\right)$.

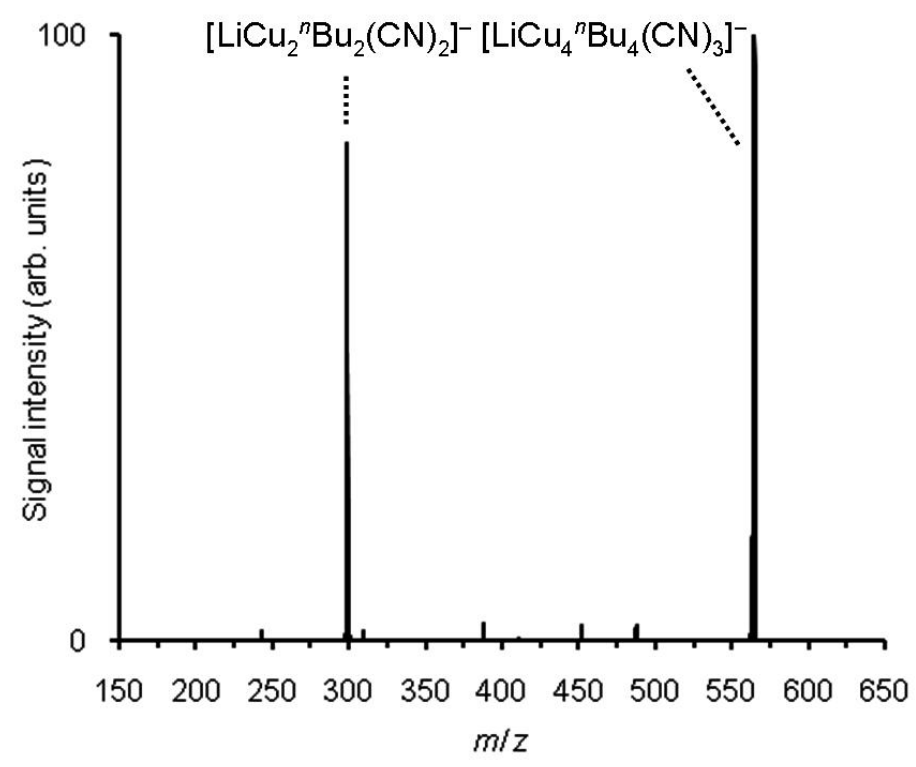

Figure S98. Mass spectrum of mass-selected $\mathrm{LiCu}_{4}{ }^{n} \mathrm{Bu}_{4}(\mathrm{CN})_{3}{ }^{-}(m / z=565)$ and its fragment ions produced upon collision-induced dissociation $\left(V_{\mathrm{exc}}=0.17 \mathrm{~V}\right)$. 


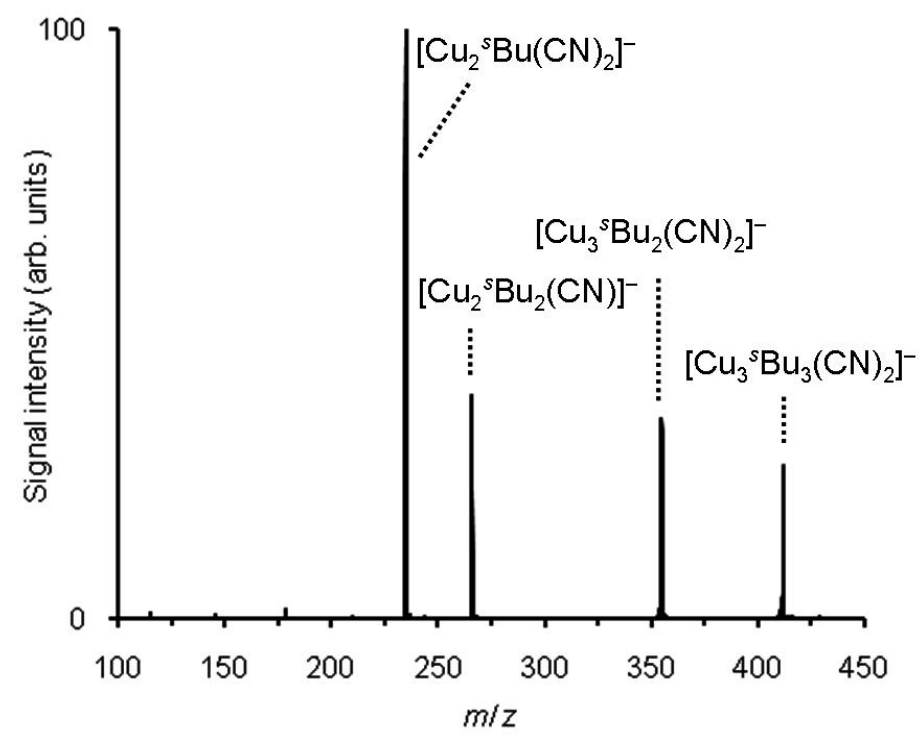

Figure S99. Mass spectrum of mass-selected $\mathrm{Cu}_{3}{ }^{s} \mathrm{Bu}_{3}(\mathrm{CN})_{2}^{-}(m / z=412)$ and its fragment ions produced upon collision-induced dissociation $\left(V_{\mathrm{exc}}=0.17 \mathrm{~V}\right)$.

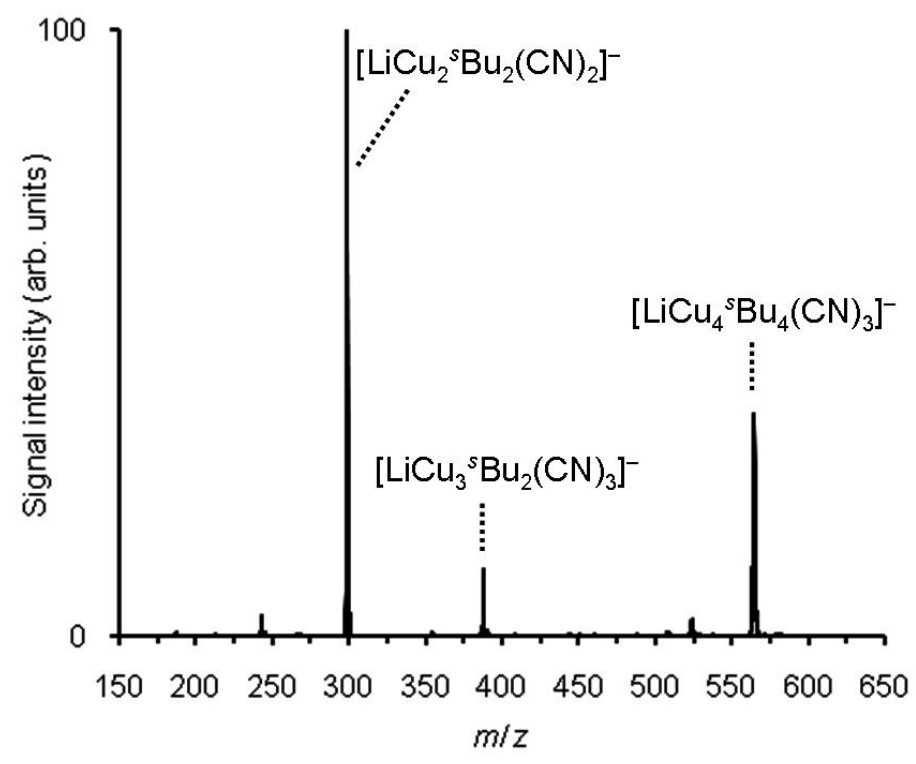

Figure S100. Mass spectrum of mass-selected $\mathrm{LiCu}_{4}{ }^{5} \mathrm{Bu}_{4}(\mathrm{CN})_{3}{ }^{-}(\mathrm{m} / z=565)$ and its fragment ions produced upon collision-induced dissociation $\left(V_{\mathrm{exc}}=0.17 \mathrm{~V}\right)$. 